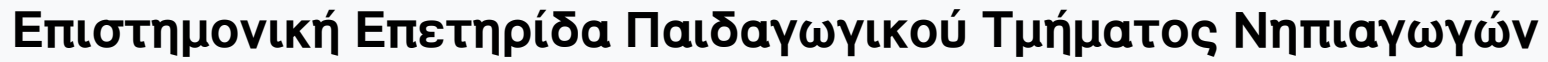

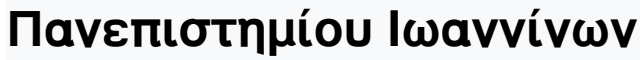

Tóp. 7 (2014)

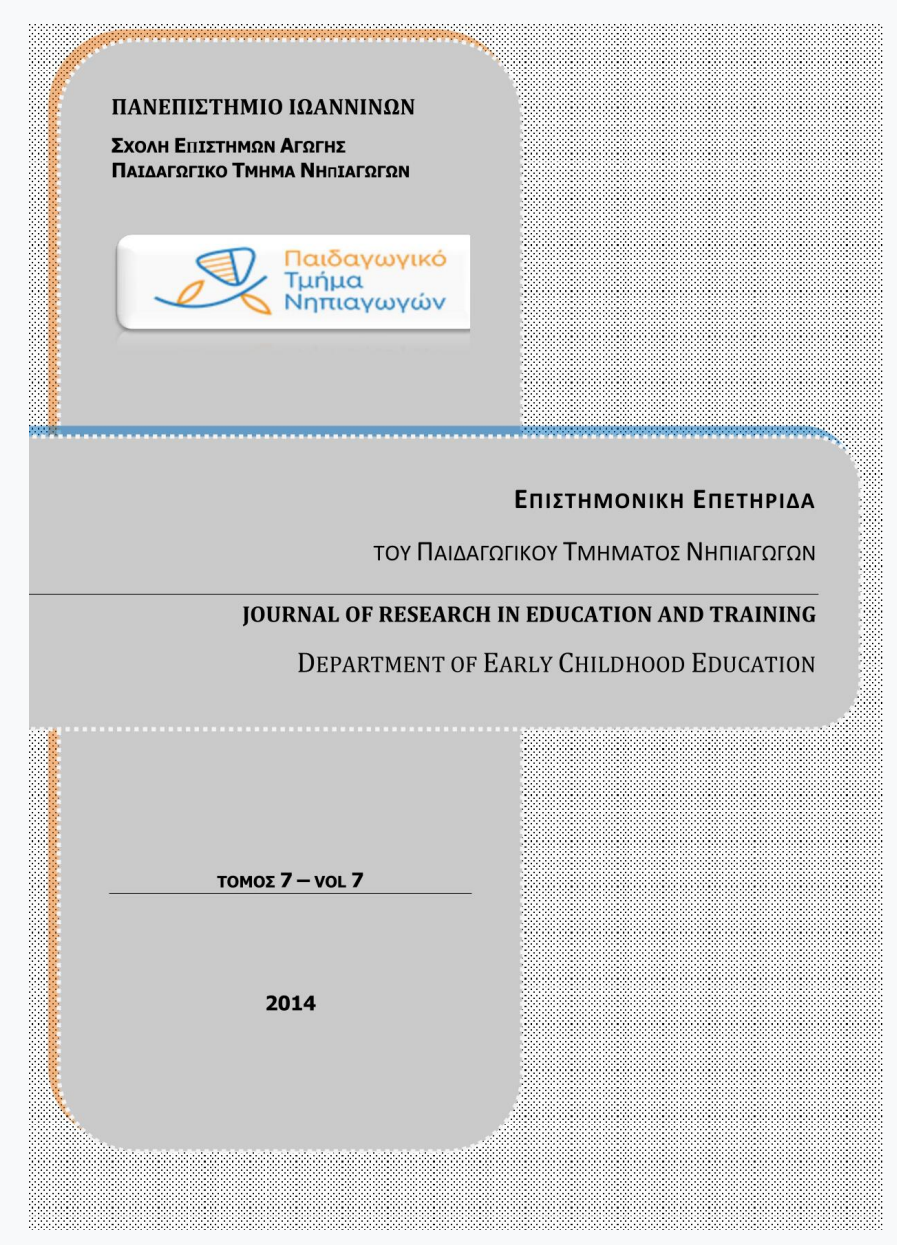

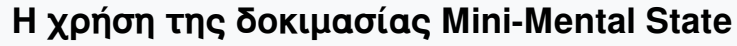

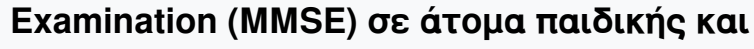

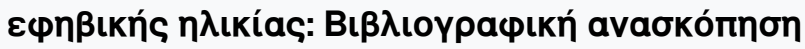
$\varepsilon \rho \varepsilon U V \eta \tau / K \omega ́ v ~ \delta \varepsilon \delta о \mu \varepsilon ́ v \omega V$
\end{abstract}

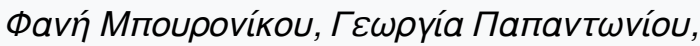

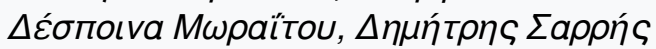

doi: $\underline{10.12681 / \text { jret. } 850}$

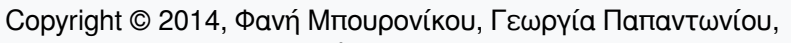

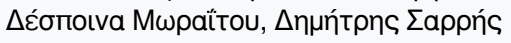

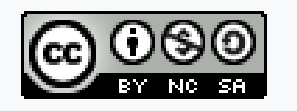

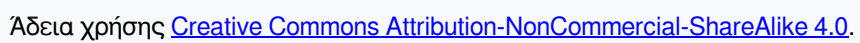

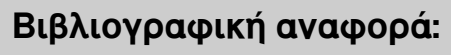

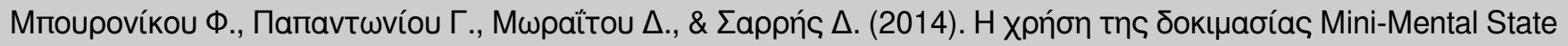

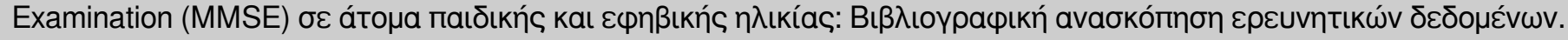

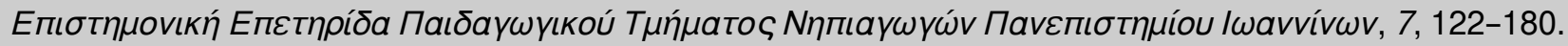
https://doi.org/10.12681/jret.850 


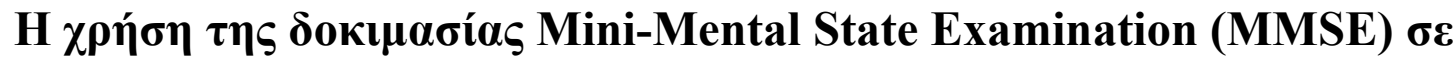

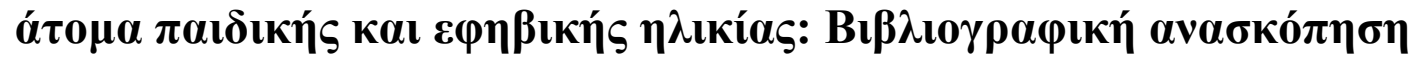

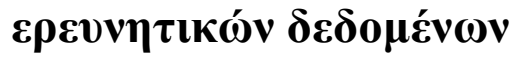

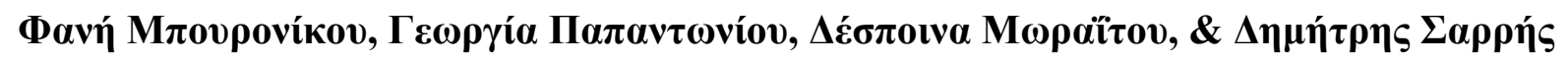

\section{ПЕРІАНЧН}

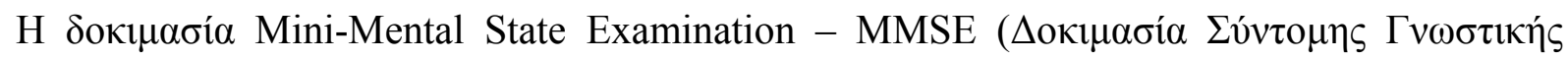

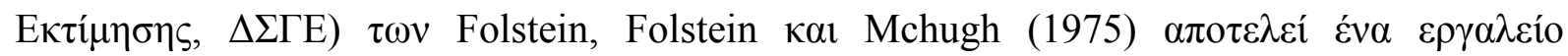

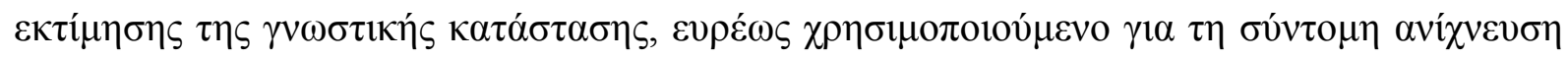

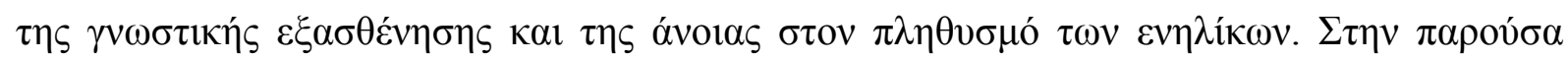

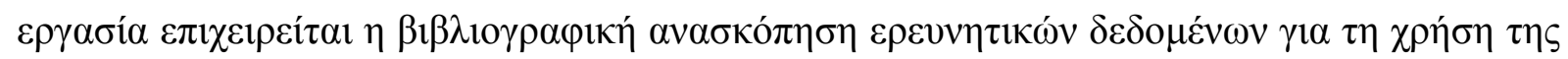

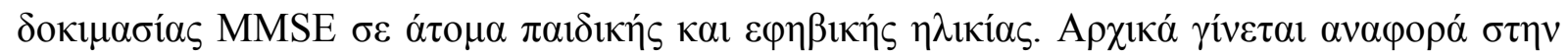

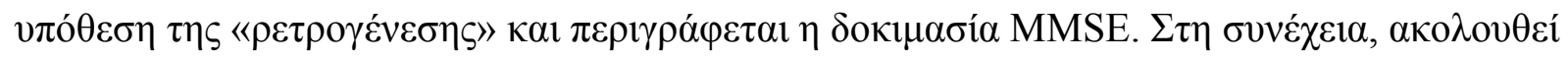

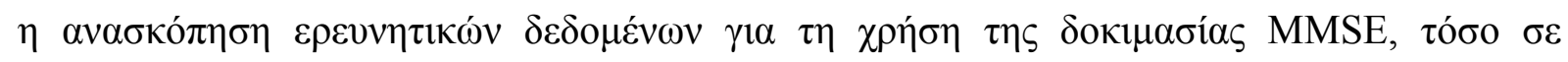

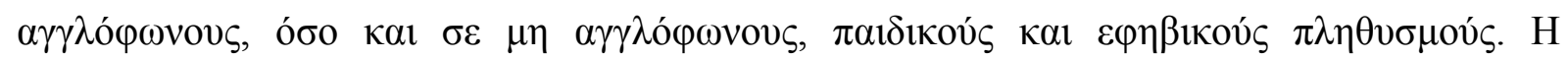

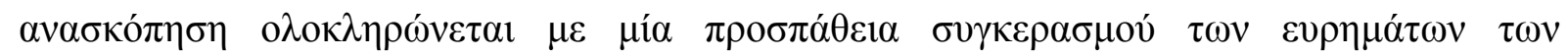

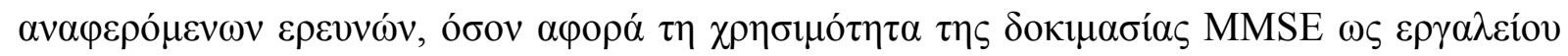

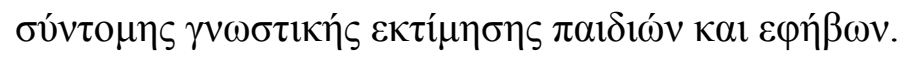

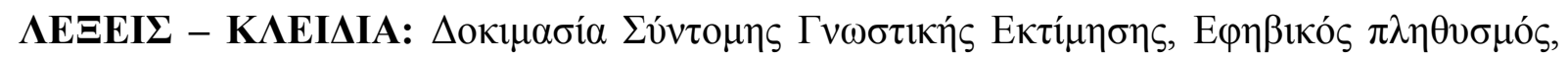

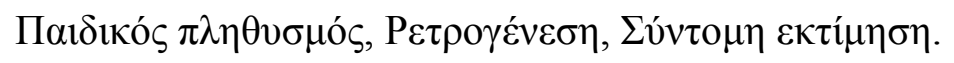




\title{
The use of the Mini-Mental State Examination in child and adolescent populations: A literature review of research data
}

\author{
Fani Bouronikou, Georgia Papantoniou, Despina Moraitou, \& Dimitris Sarris
}

\begin{abstract}
The Mini-Mental State Examination (MMSE; Folstein, Folstein, \& Mchugh, 1975) is a cognitive status test widely used to screen cognitive impairment and dementia in the adult population. This paper attempts a literature review of research data about the use of the MMSE in child and youth populations. At first, the paper refers to the hypothesis of "retrogenesis" and the description of the MMSE is provided. The paper continues with the review of research data about the use of the MMSE, both in English and non-English speaking populations including children and adolescents. The review concludes with an attempt to reconcile the findings of the research mentioned regarding the usefulness of the MMSE as a cognitive screening tool for children's and adolescents' development.
\end{abstract}

KEY-WORDS: Mini-Mental State Examination, Adolescent population, Child population, Retrogenesis, Screening test. 


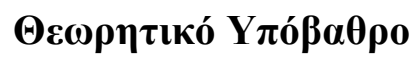

$H v \pi \delta ́ \theta \varepsilon \sigma \eta \tau \eta \varsigma ~ « \rho \varepsilon \tau \rho o \gamma \varepsilon ́ v \varepsilon \sigma \eta \varsigma »$

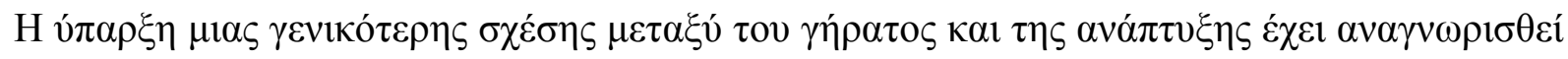

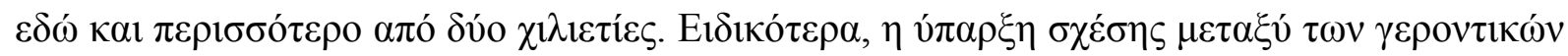

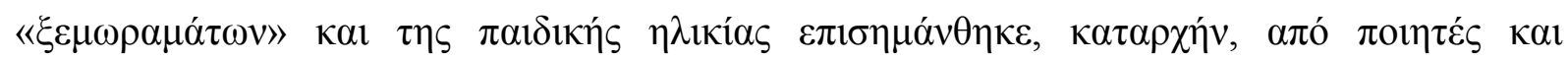

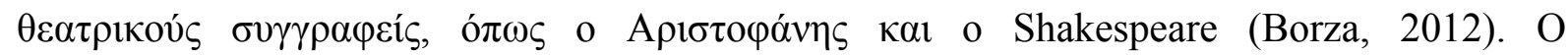
A

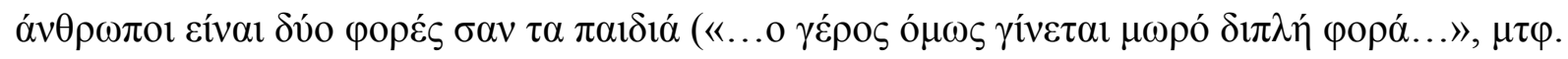

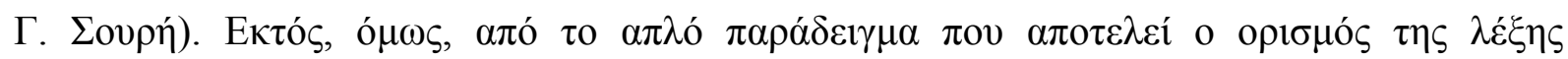

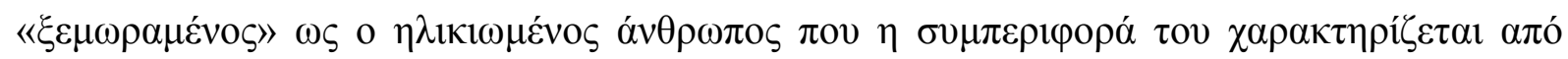

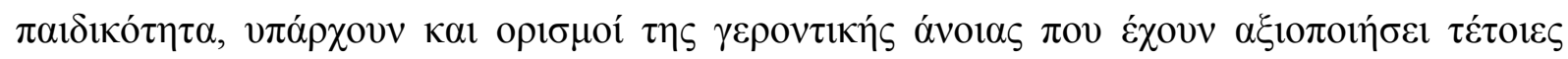

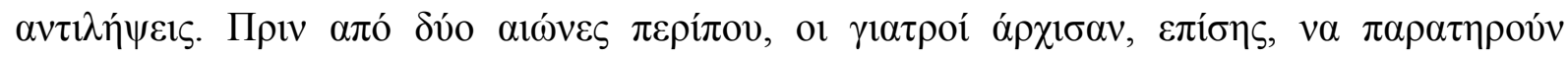

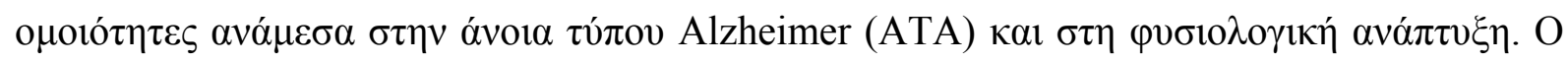

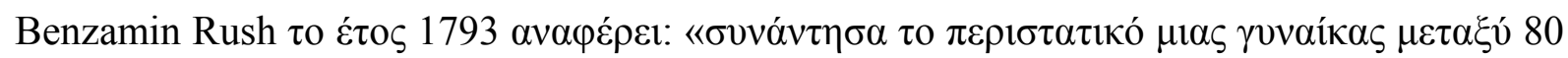

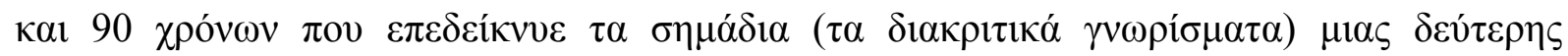
$\beta \rho \varepsilon \varphi ı$

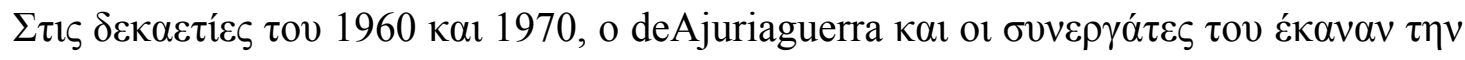

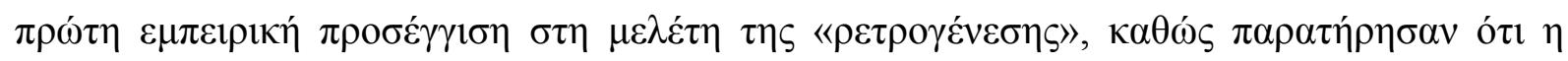

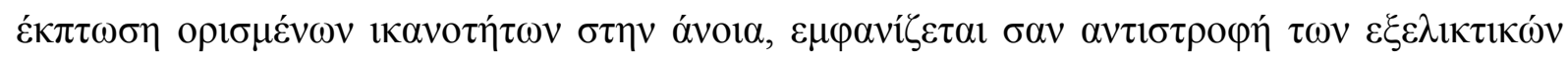

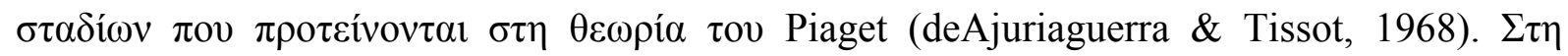

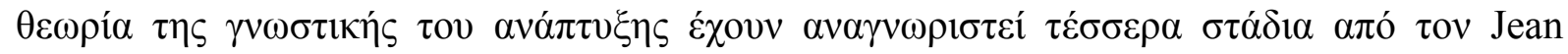

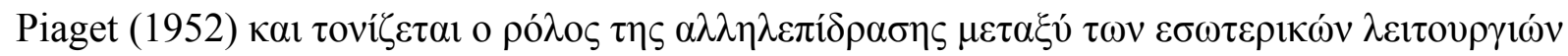

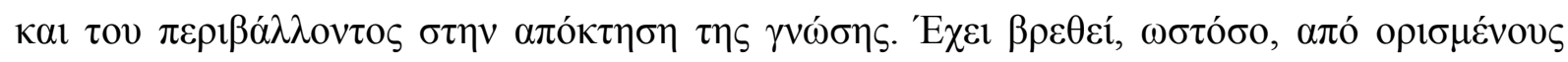

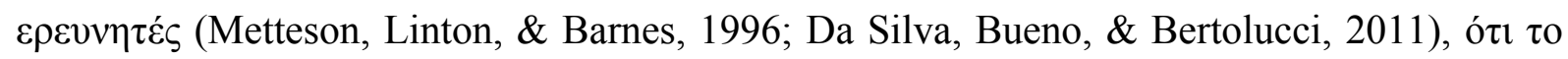




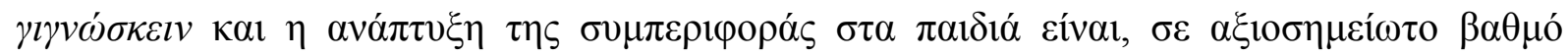

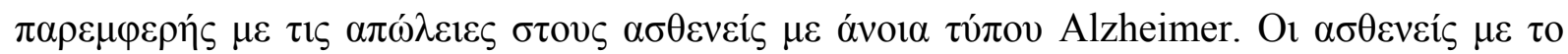

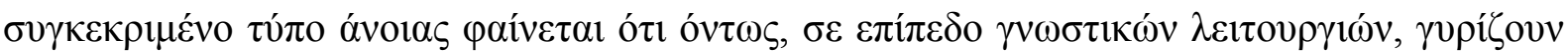

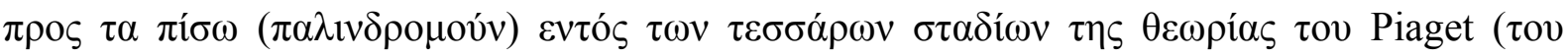

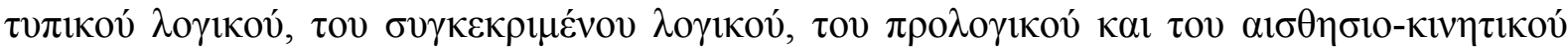
$\sigma \tau \alpha \delta i ́ o v)$.

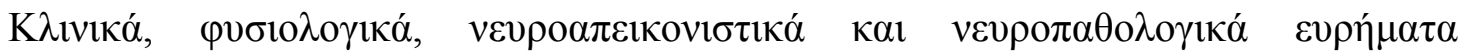

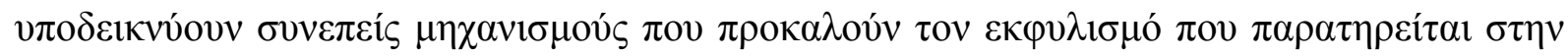

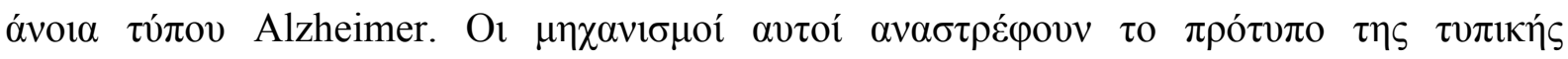
(

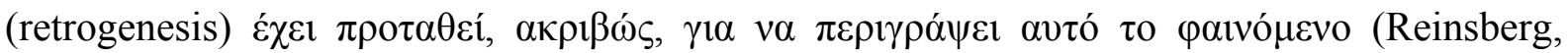
Franssen, Hasan, Monteiro, Boksay, Souren et al., 1999a. Reinsberg, Kenowsky, Franssen,

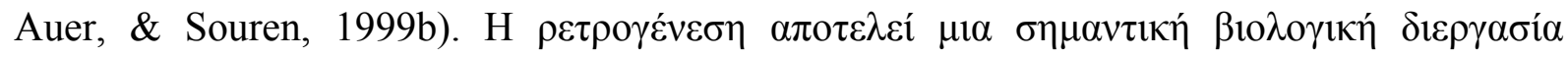

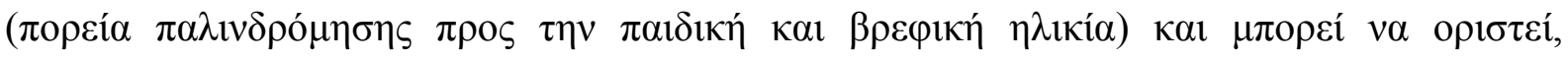

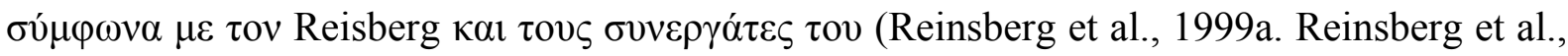

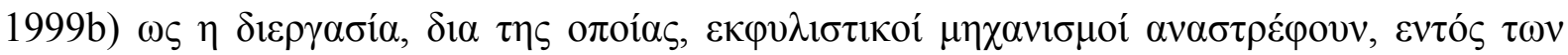

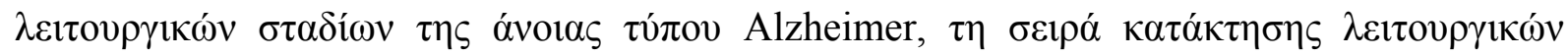

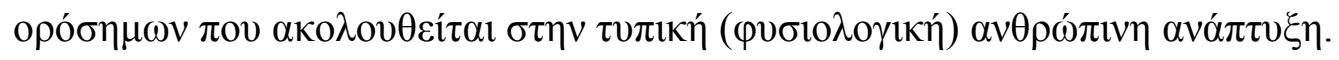

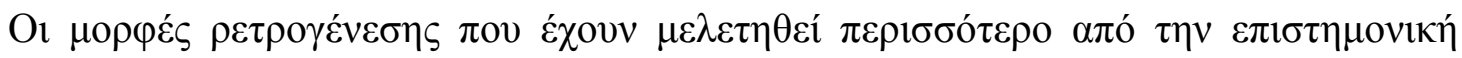

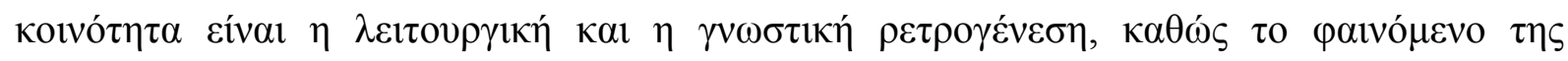

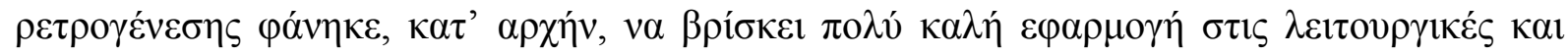

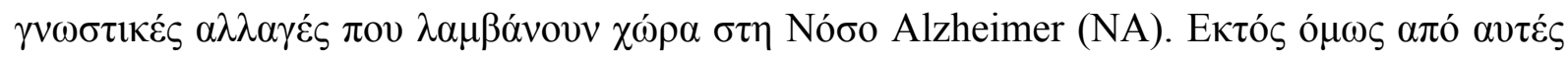

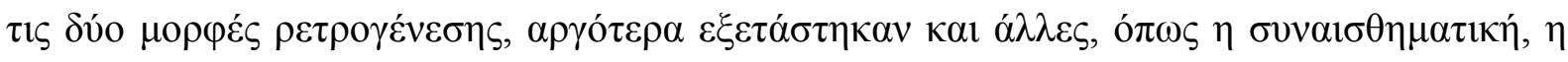

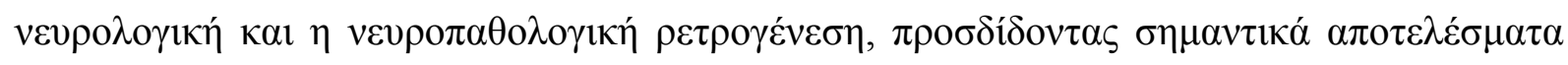
(Rudial-Alvarez, Sola, Machado, Sintas, \& Bohm, 2013). 


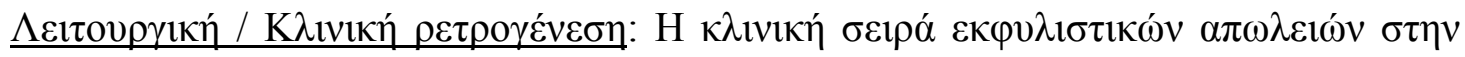

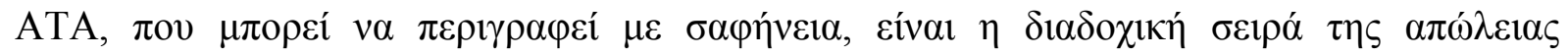

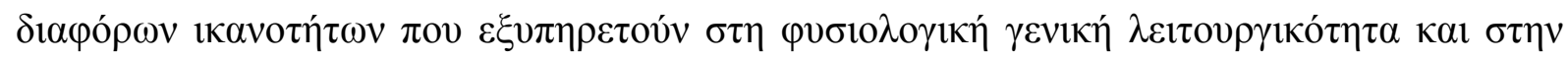

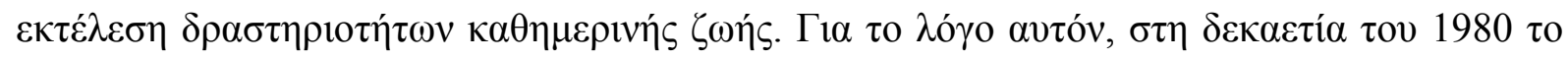

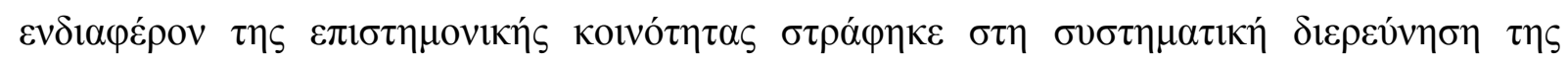

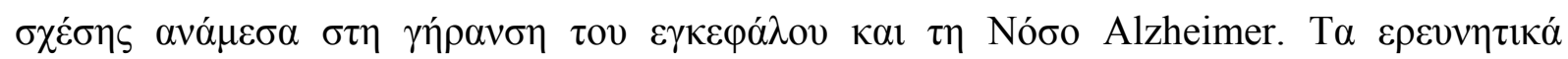

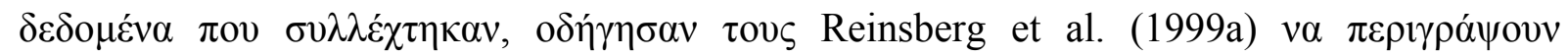

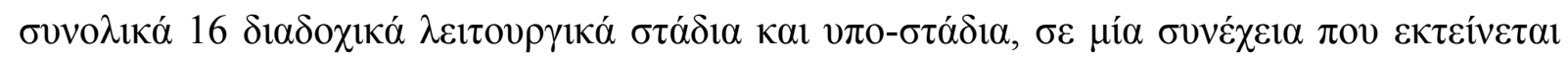

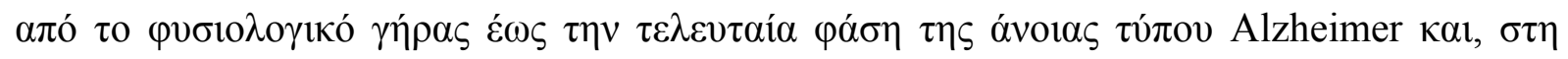

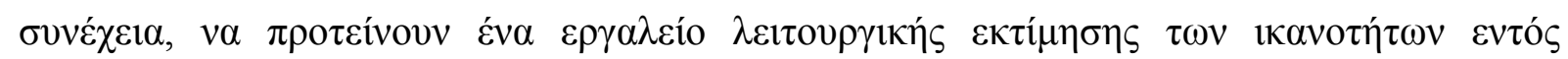

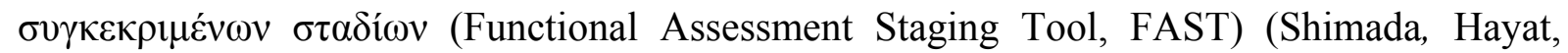
Meguro, Oo, Jafri, Yamadori et al., 2003).

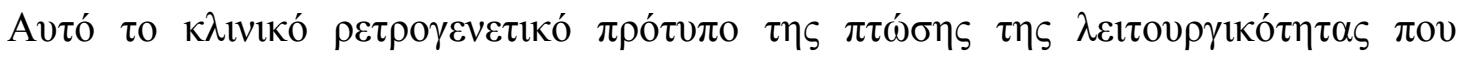

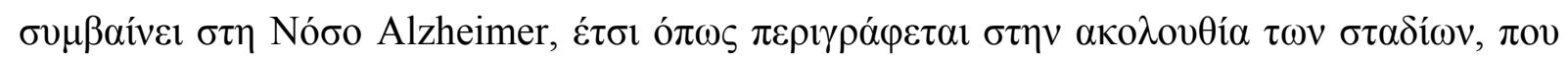

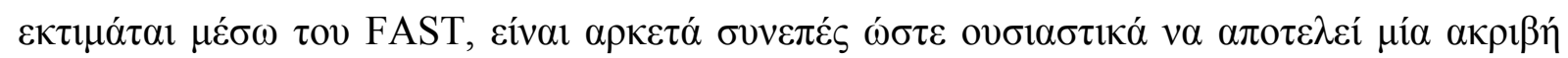

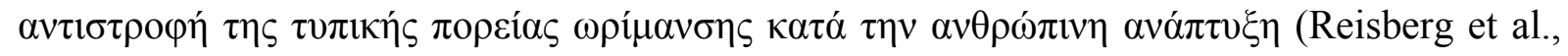
1999b. Ventirelli, Magalioni, Scarcini, \& Schena, 2012). Гı $\alpha \alpha \rho \alpha ́ \delta \varepsilon 1 \gamma \mu \alpha, \eta \delta \varepsilon \xi$ ló $\rceil \tau \alpha ~ v \alpha$

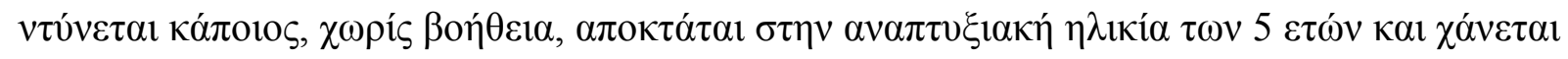

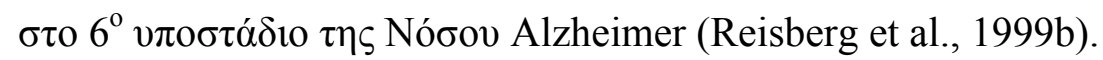

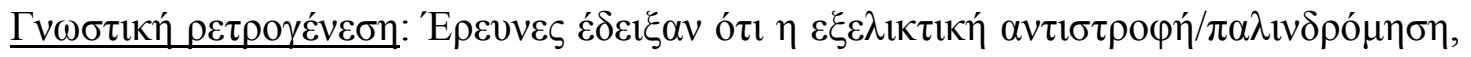

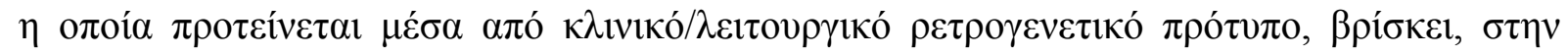

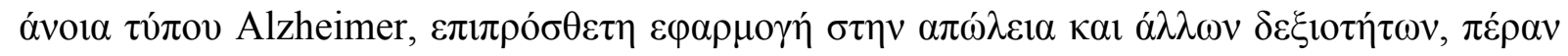

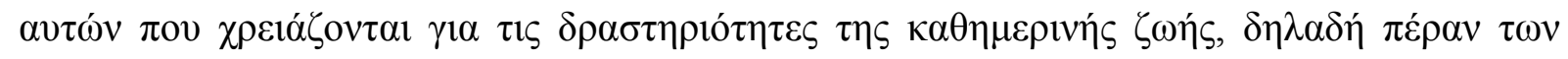

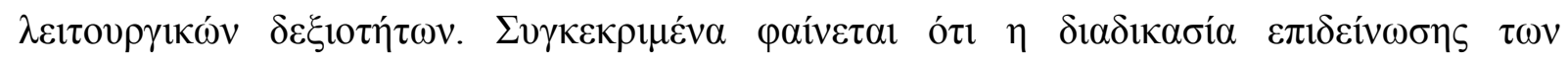

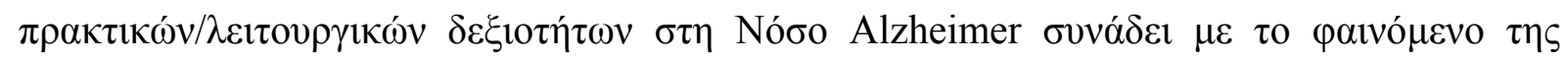




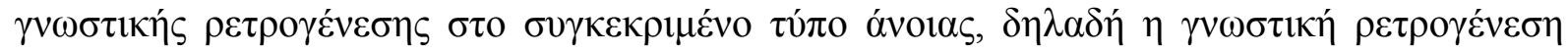

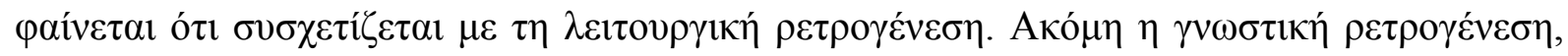

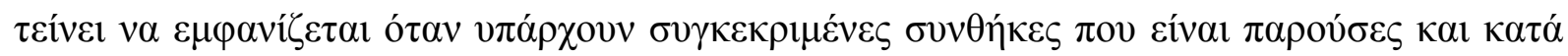

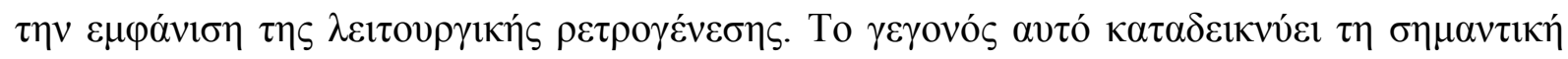

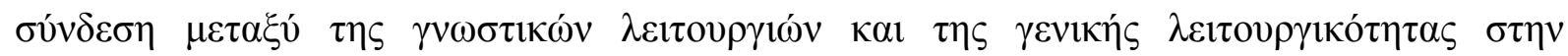

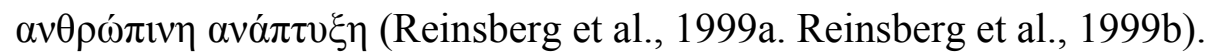

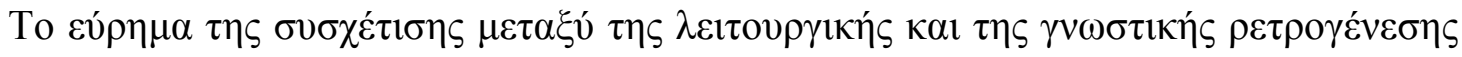

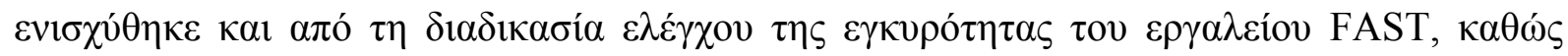

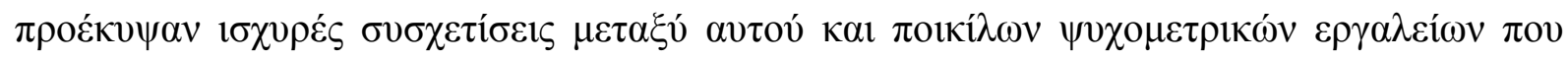

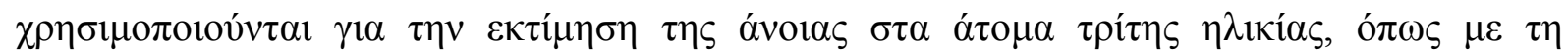

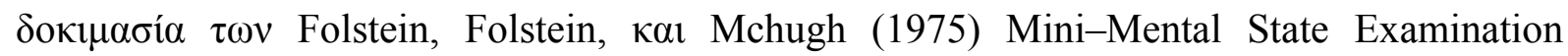
(MMSE) (Ferrario, Seccia, Massaia, Fonte \& Molaschi, 1998. Reinsberg et al., 1999a. Reinsberg et al., 1999b).

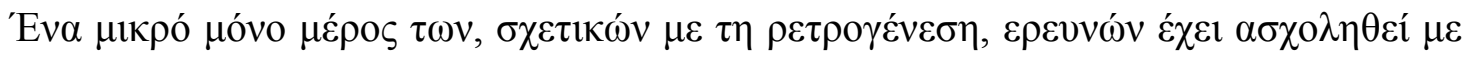

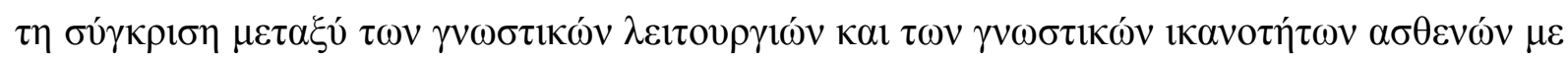

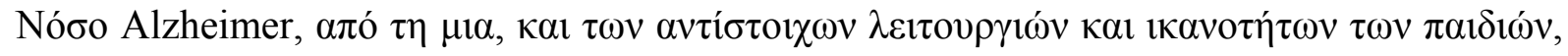

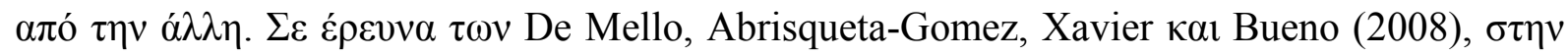

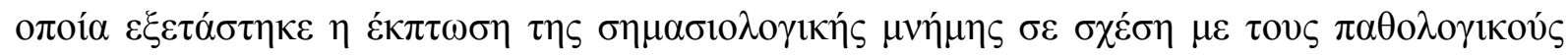

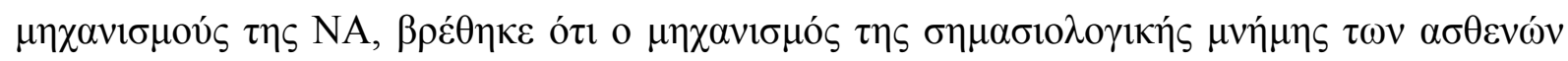

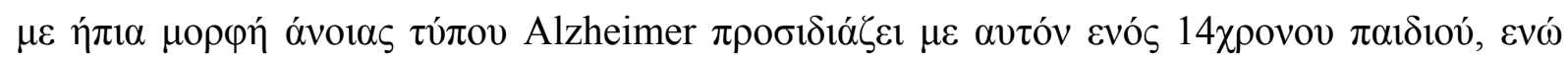

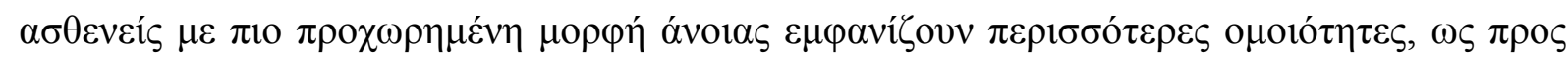

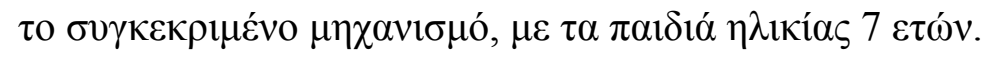

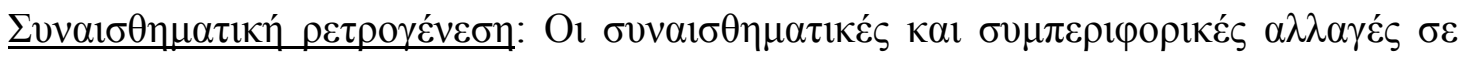

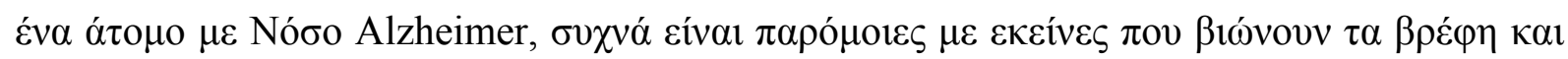

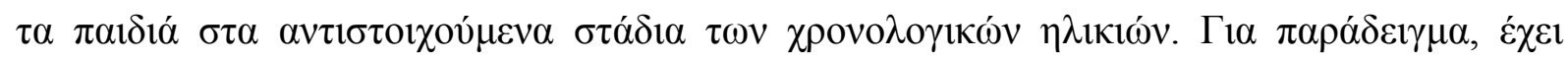




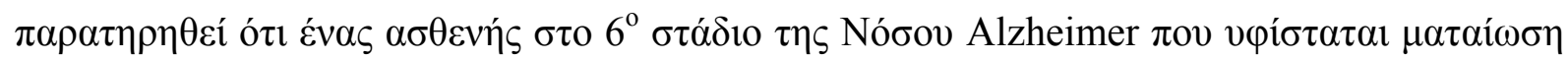

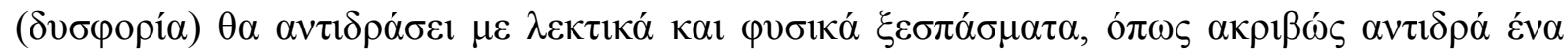

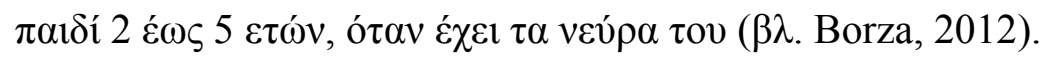

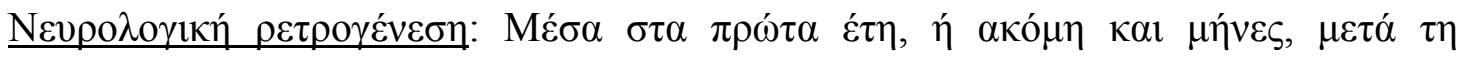

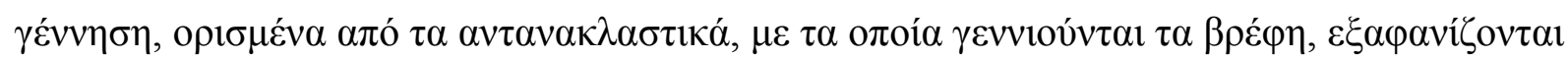

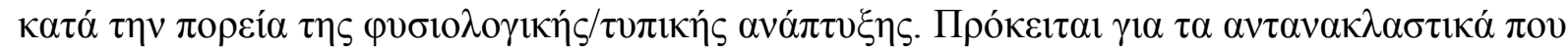

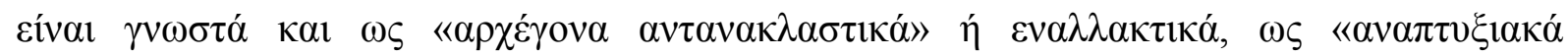

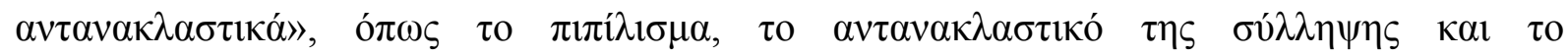

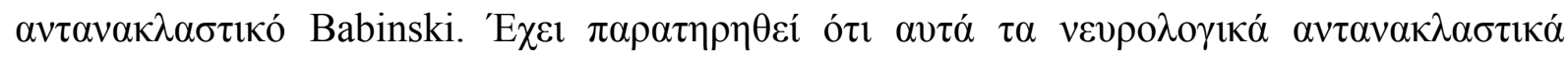

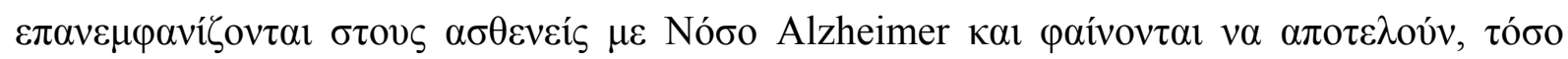

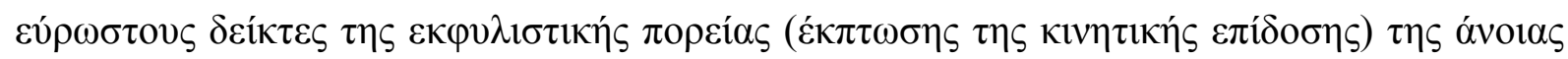

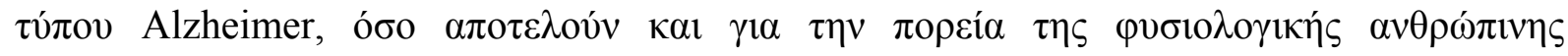

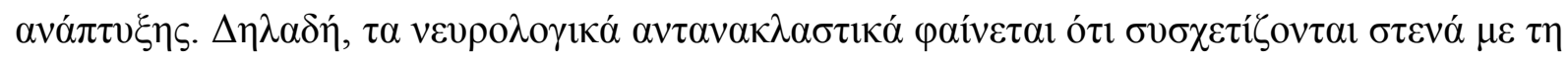

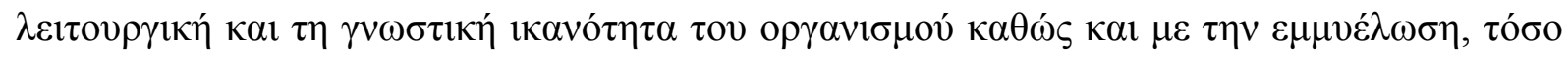

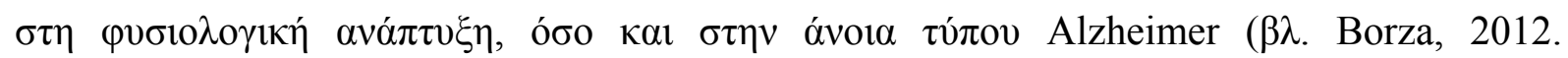
Reinsberg et al., 1999a).

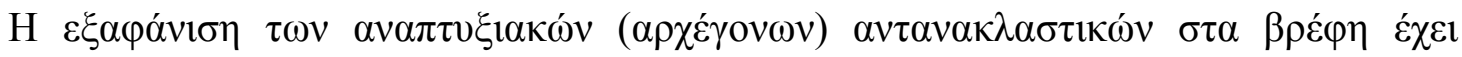

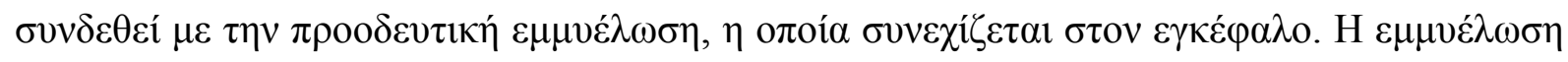

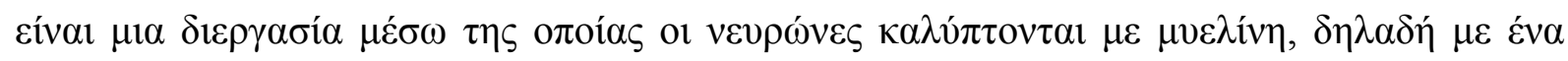

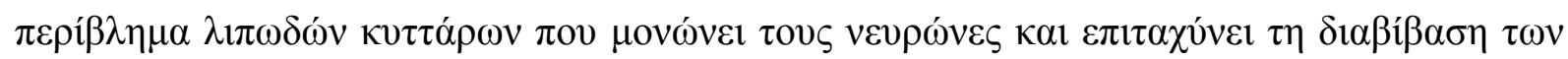

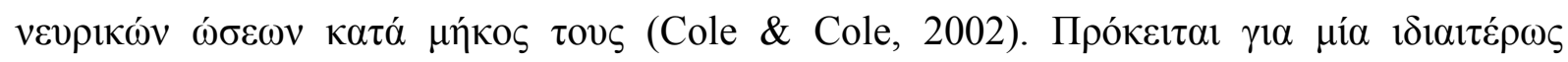

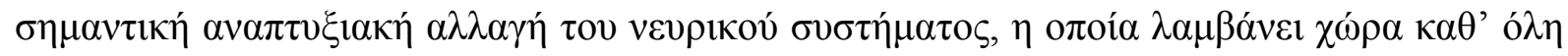

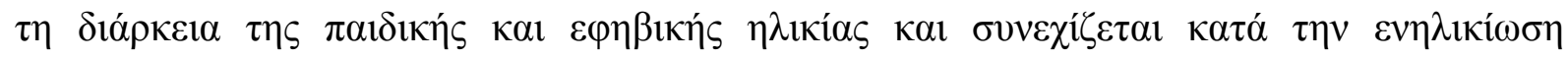

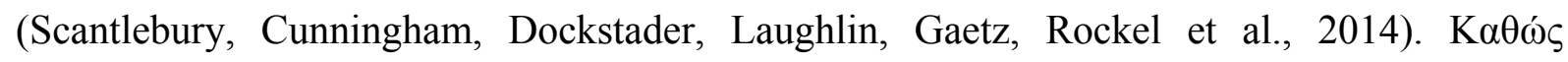

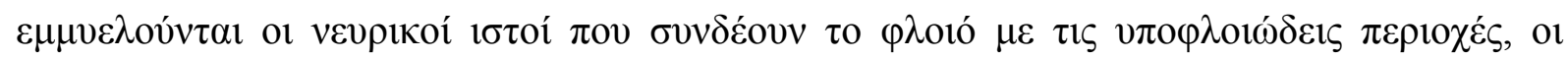




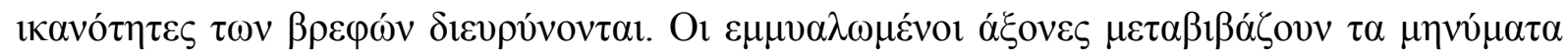

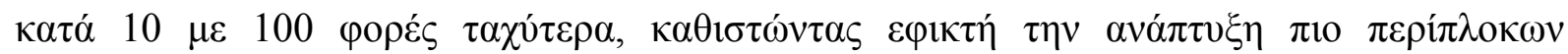

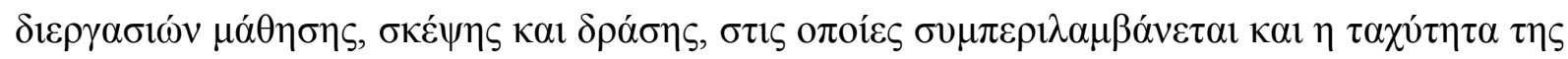

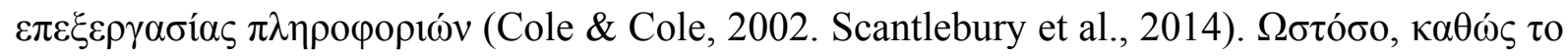

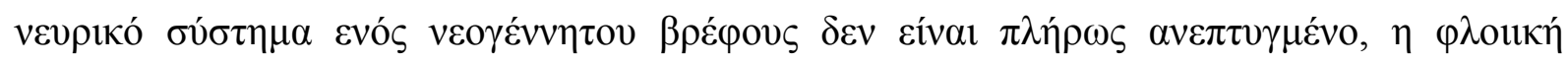

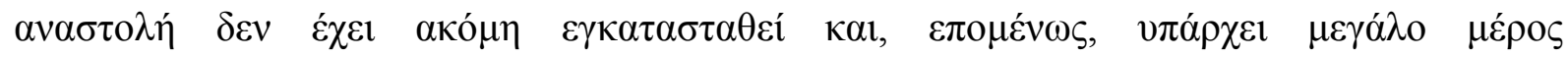

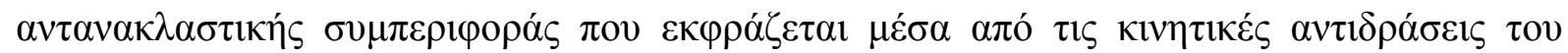

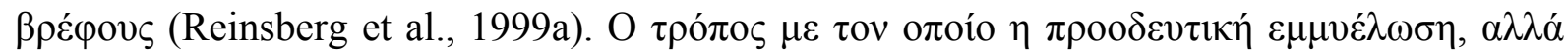

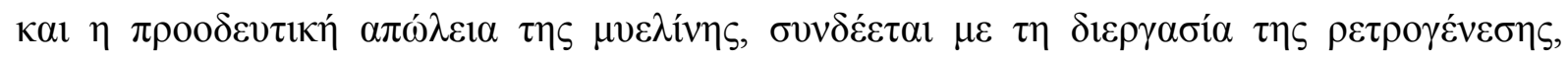

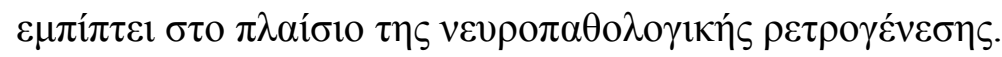

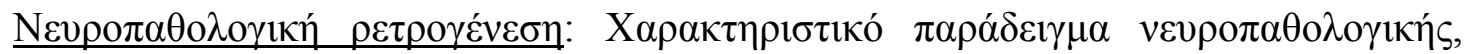

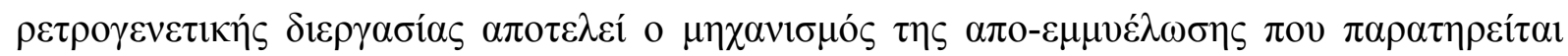

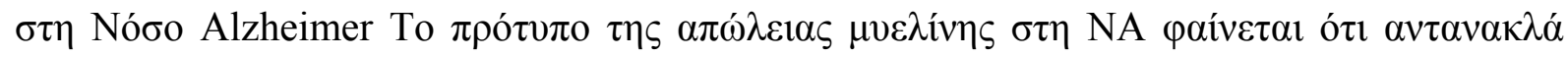

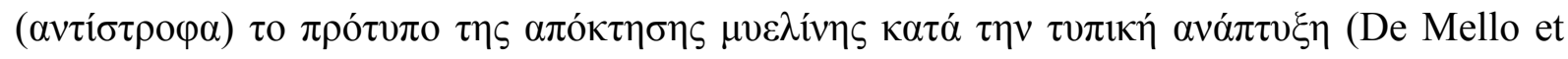

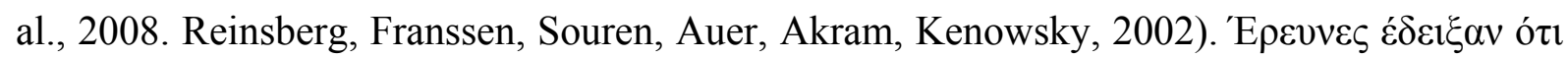

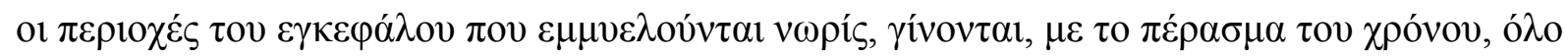

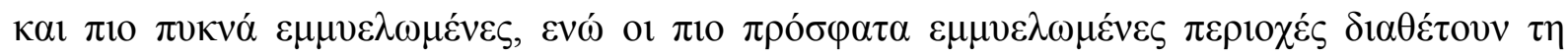

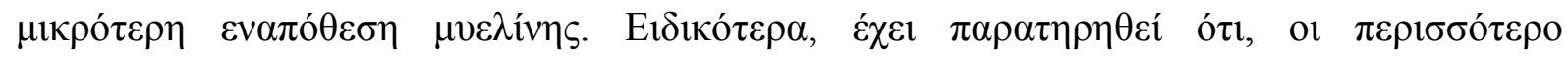

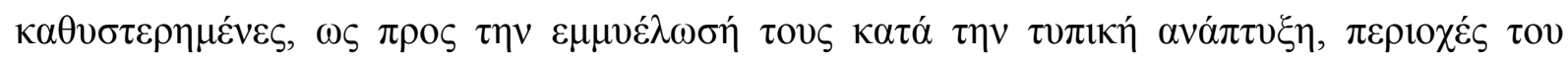

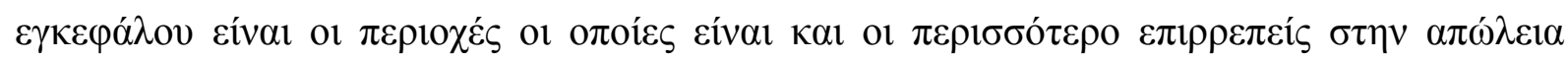

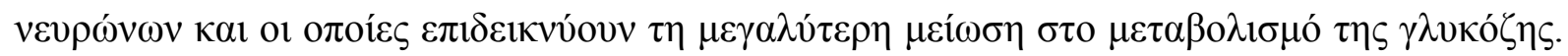

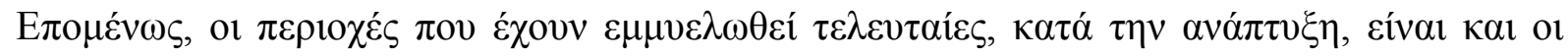

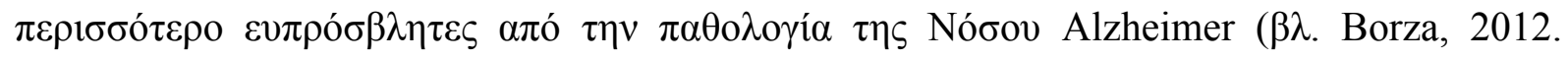

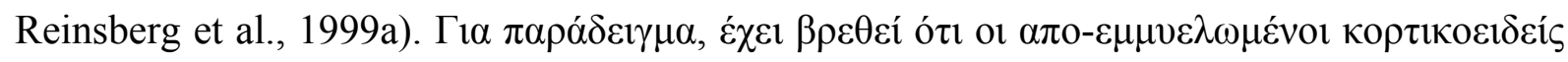

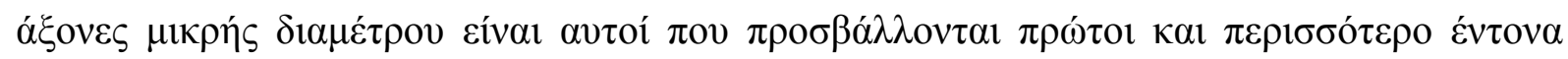




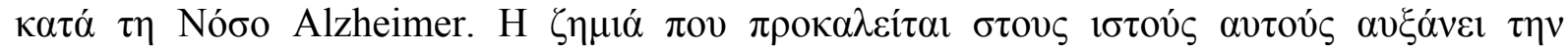

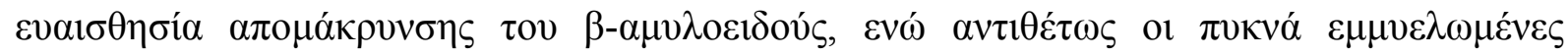

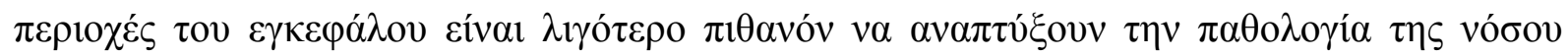
Alzheimer (Beckman \& Ames, 1998. Brickman, Meier, Korgaonkar, Provenzano, Grieve, Siedlecki et al., 2012).

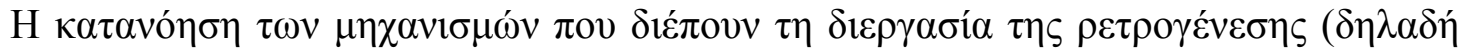

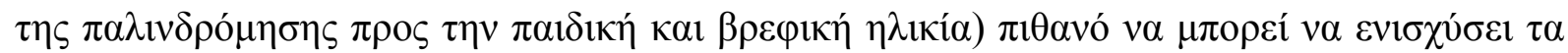

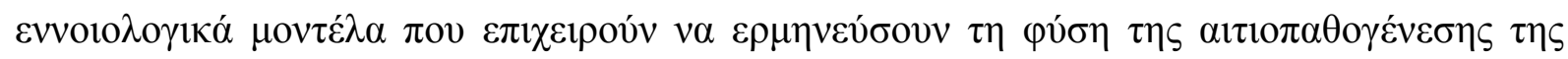

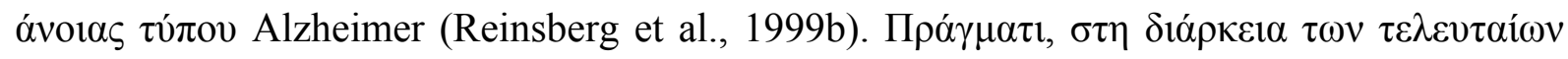

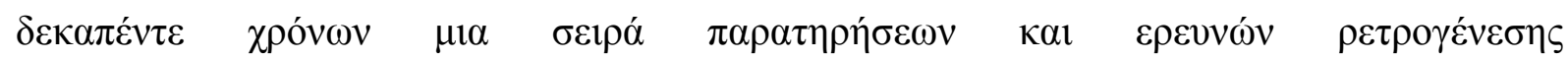

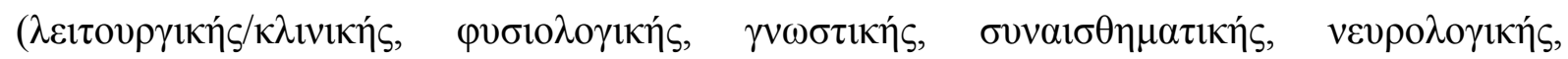

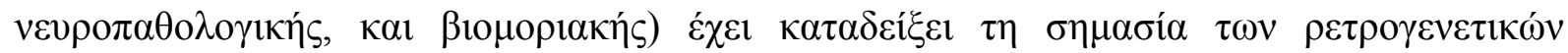

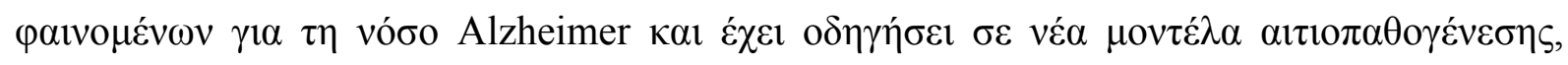

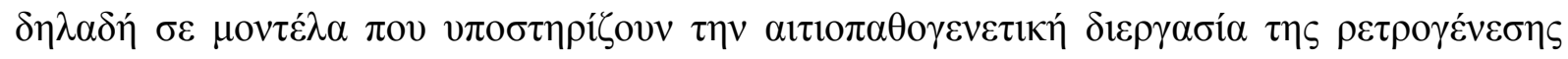

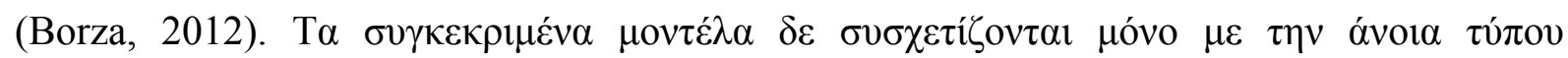

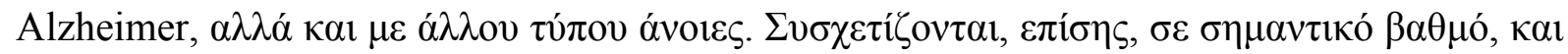

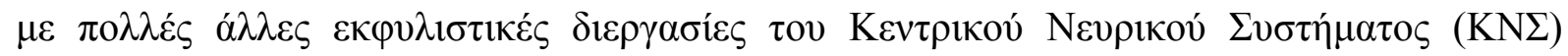
(Reinsberg et al., 1999a. Reinsberg et al., 1999b). 


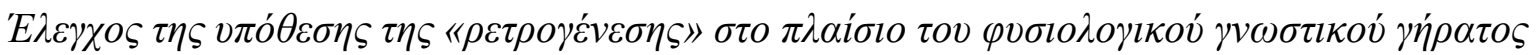

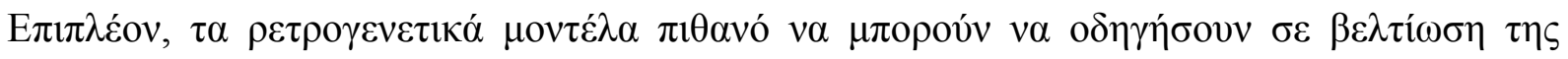

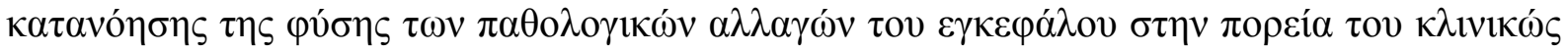

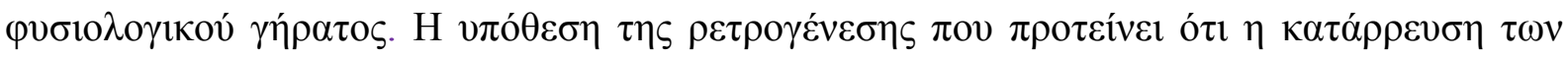

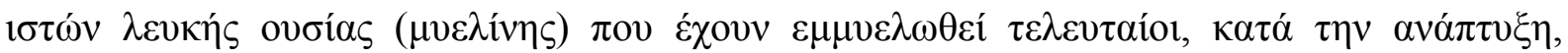

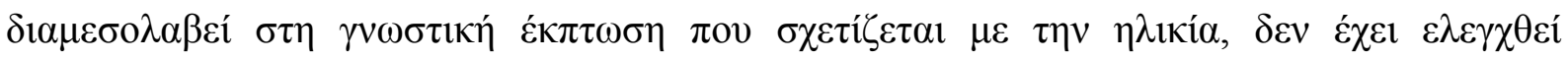

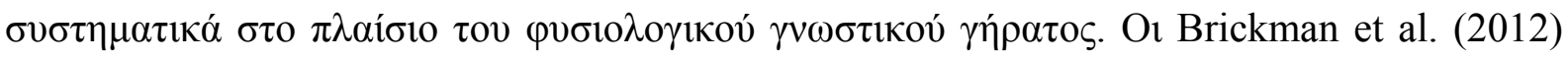

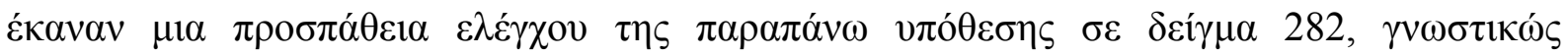


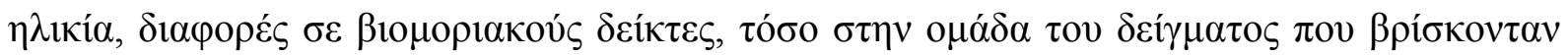

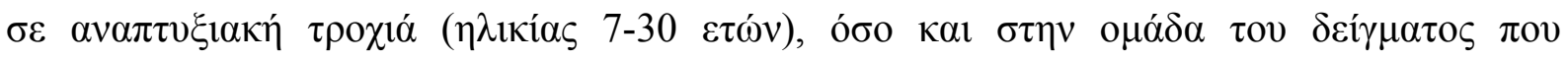

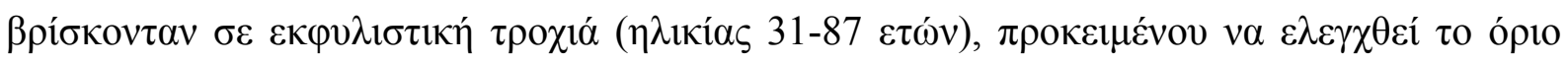

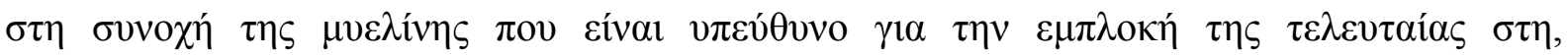

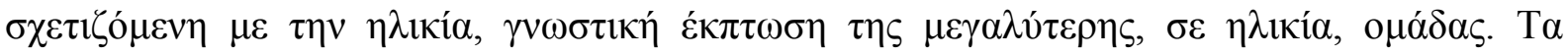

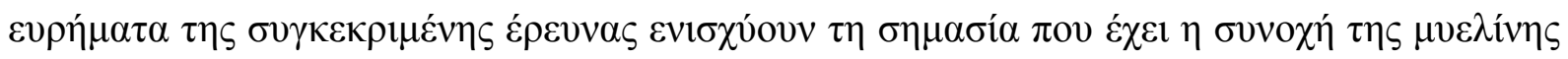

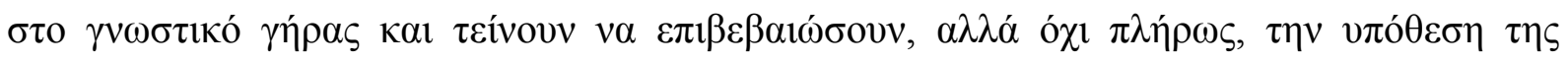

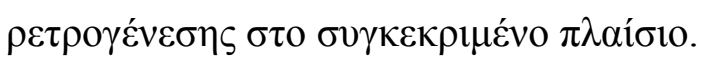

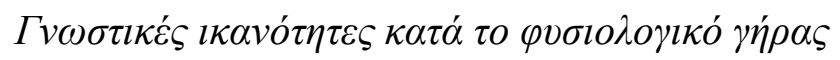

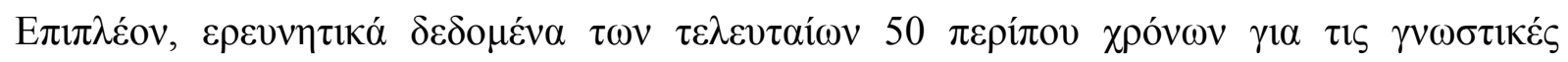

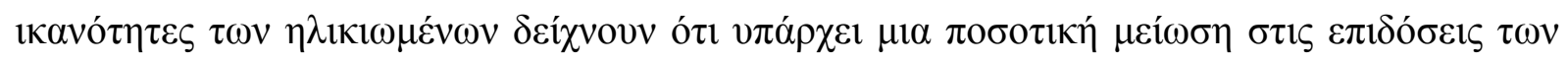

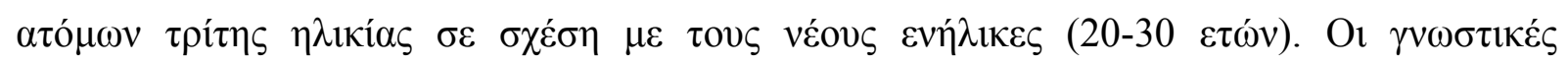




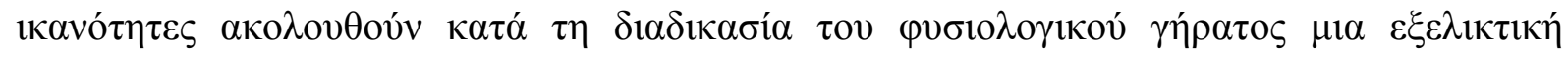

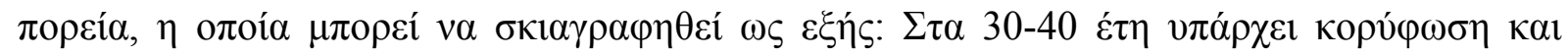

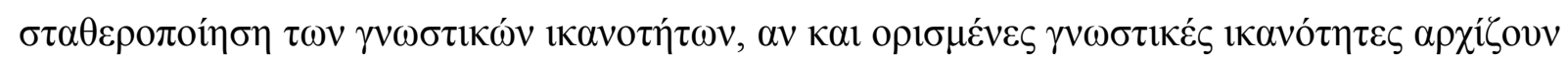

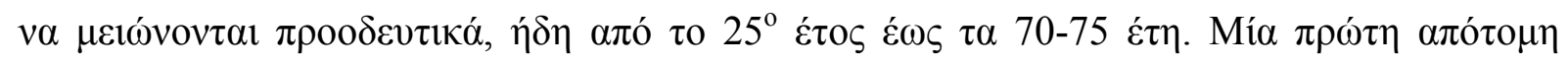

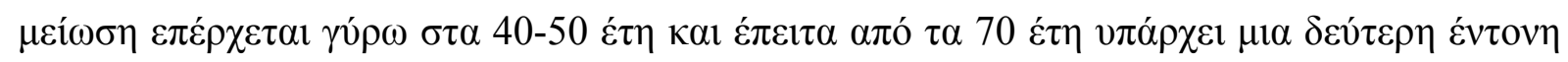

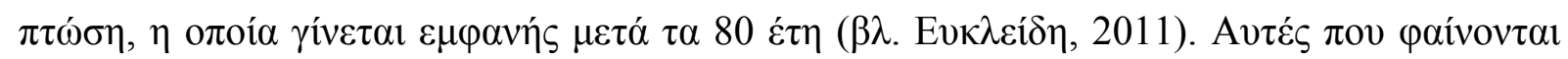

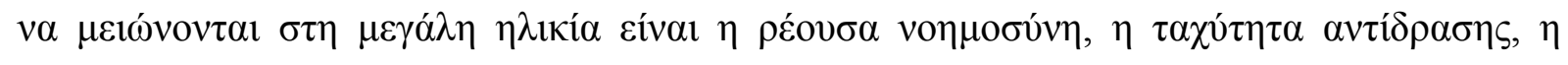

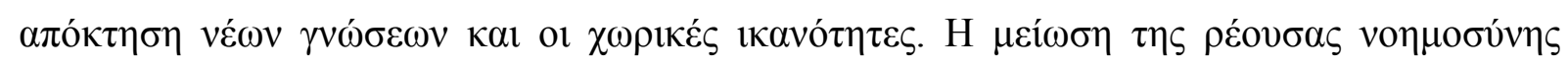

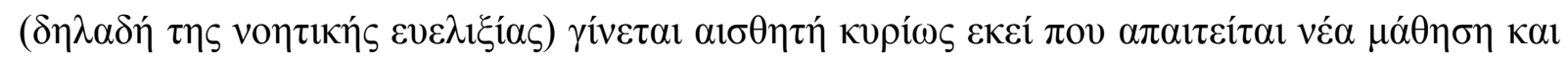

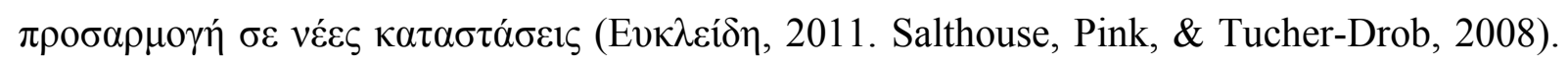

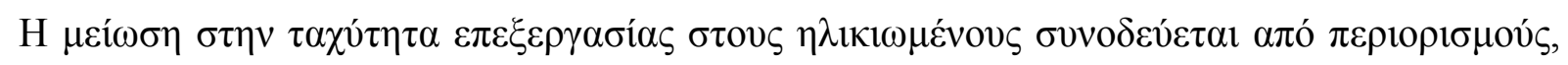

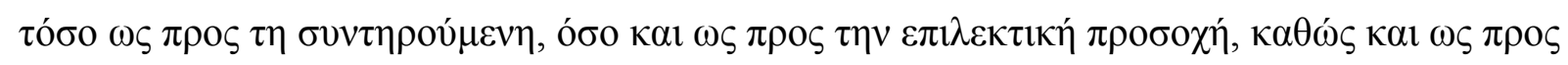

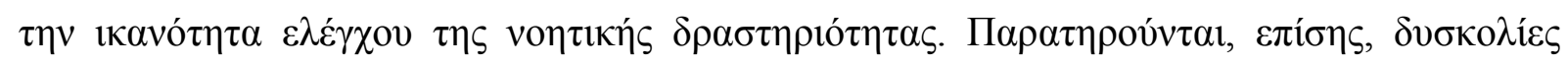

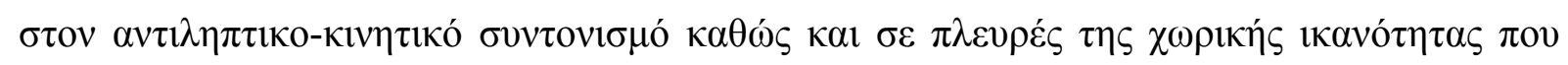

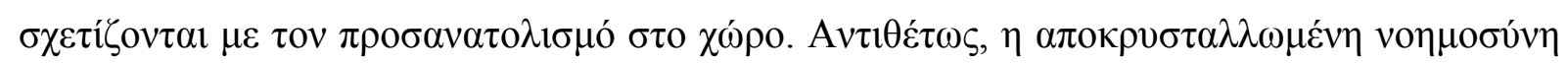

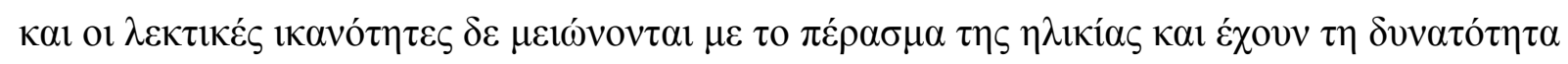

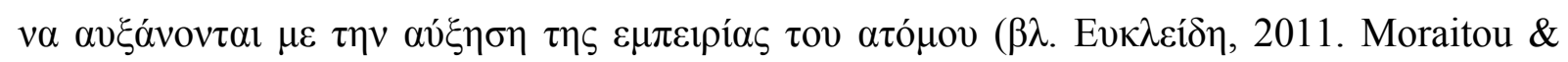
Efklides, 2012). 


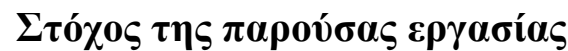

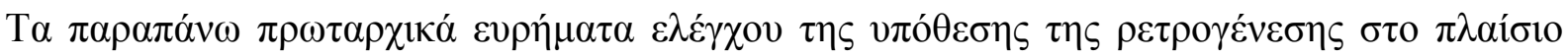

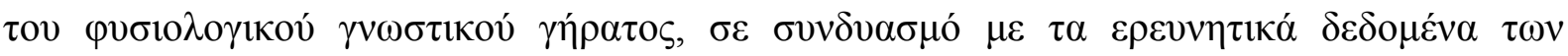

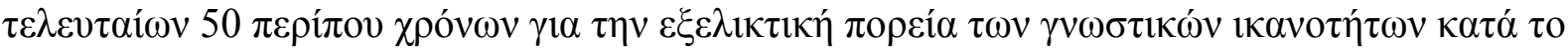

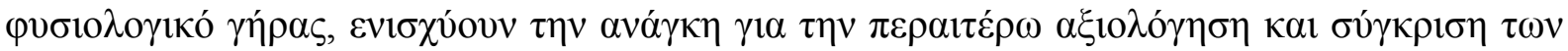

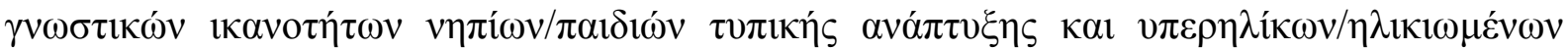

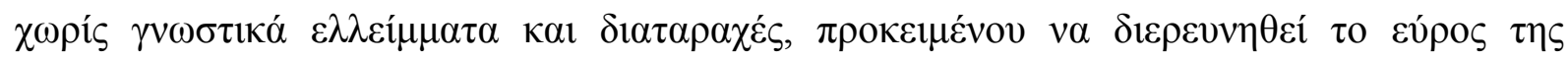

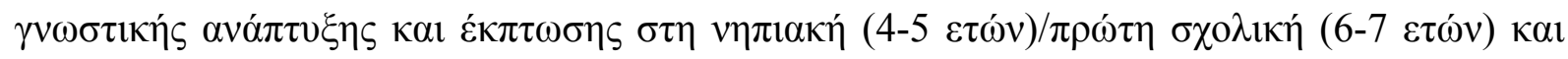

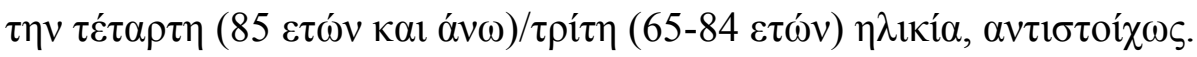

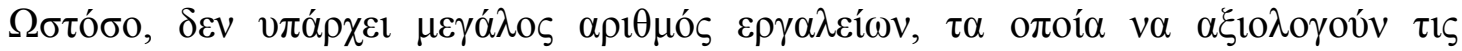

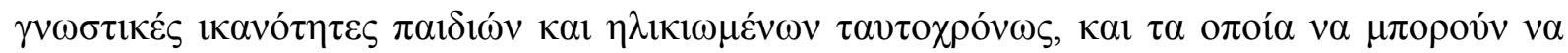

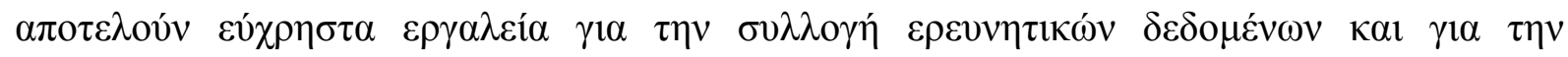

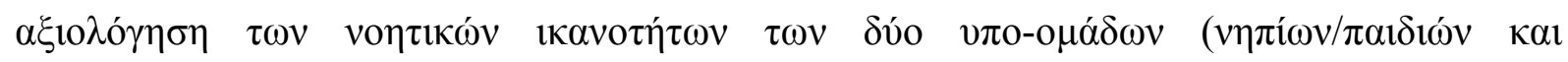
$v \pi \varepsilon \rho \eta \lambda i ́ \kappa \omega v / \eta \lambda \imath \kappa \imath \omega \mu \varepsilon ́ v \omega v)$.

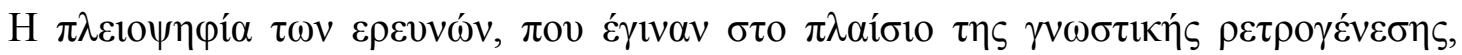

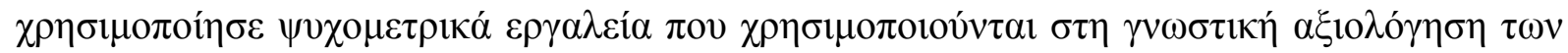

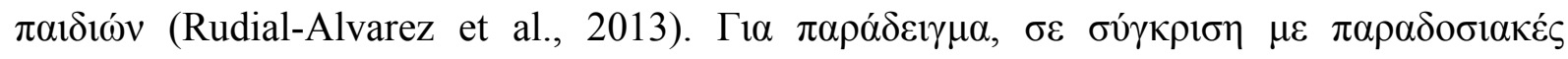

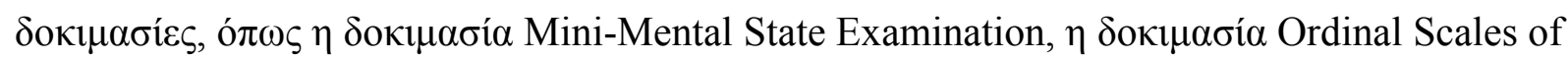

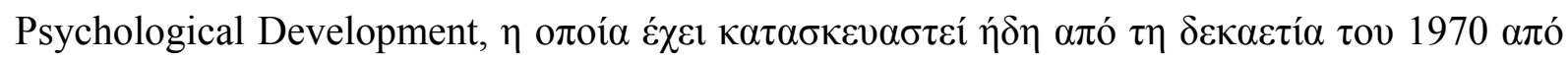

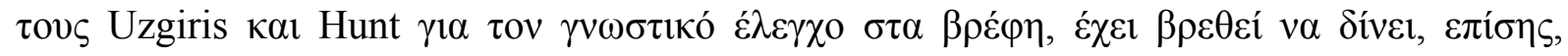

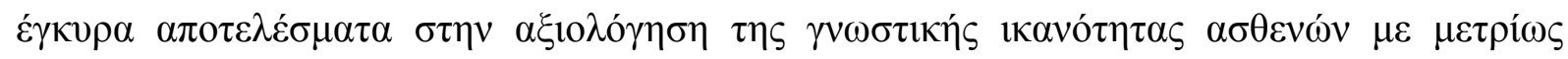

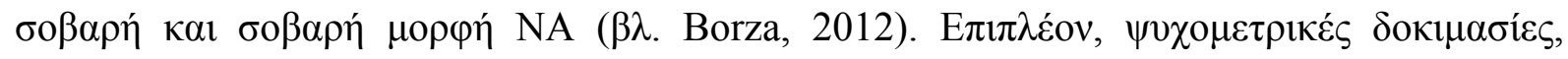

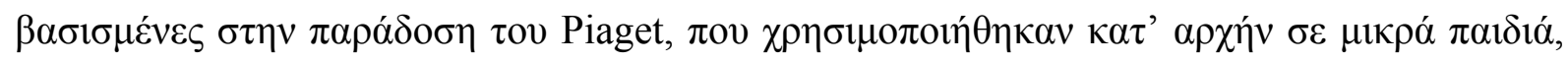

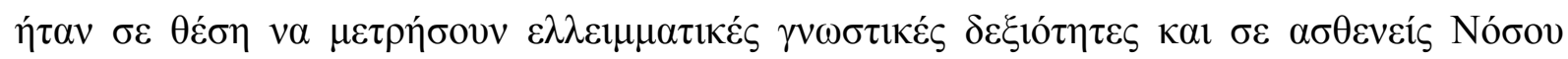




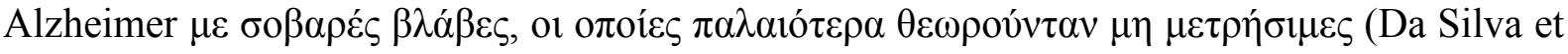

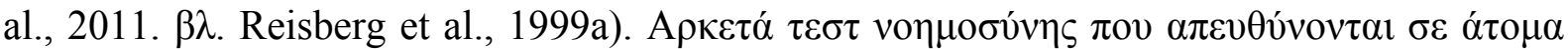

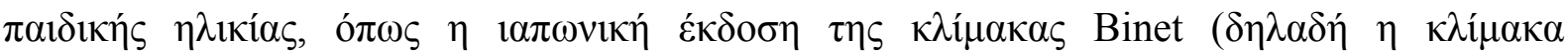

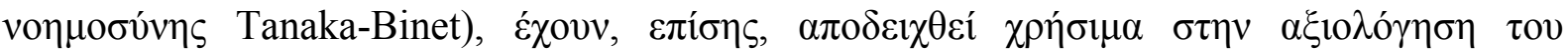

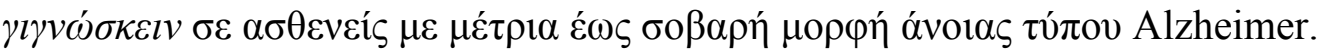

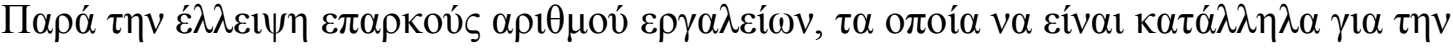

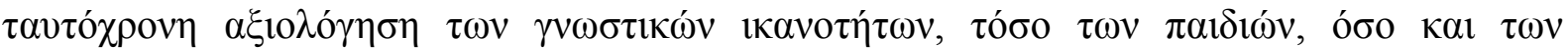

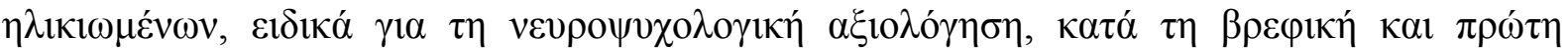

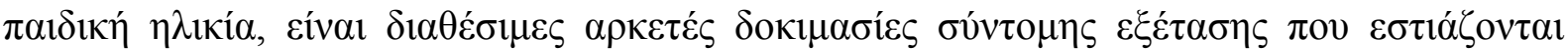

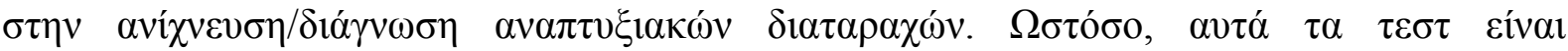

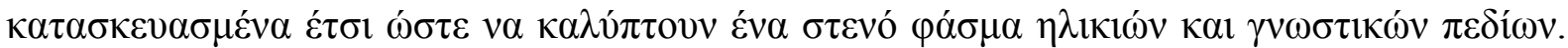

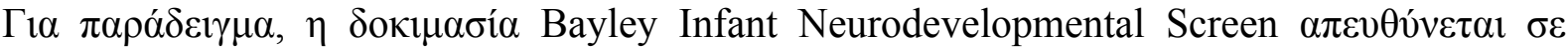

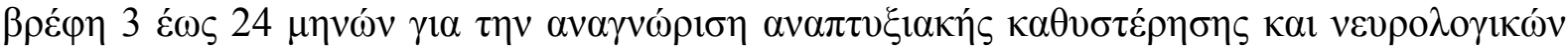

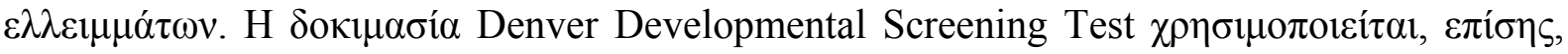

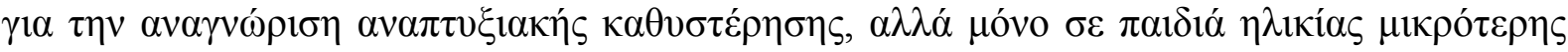

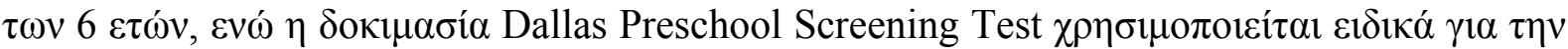

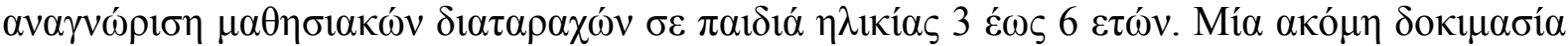

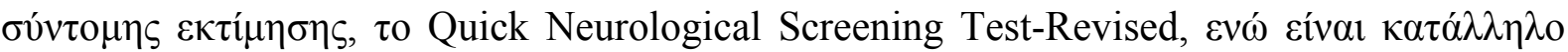

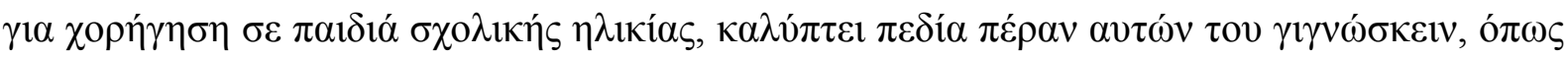

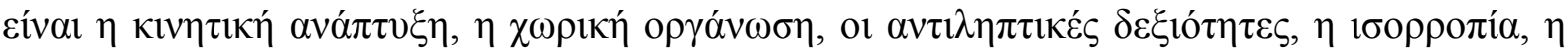

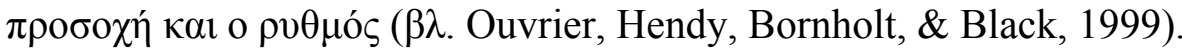

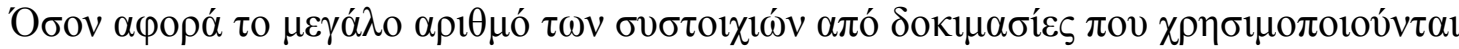

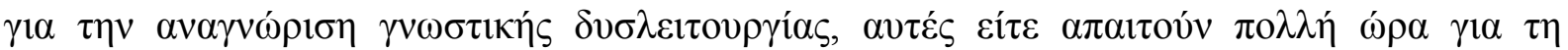

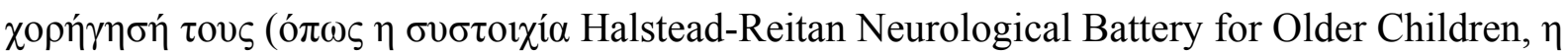

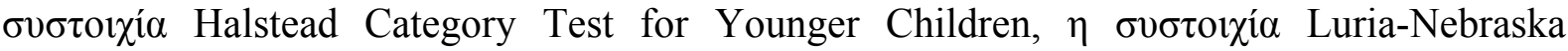




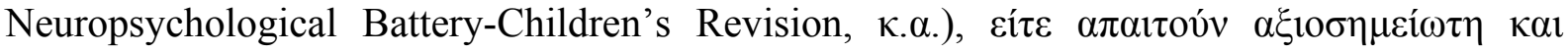

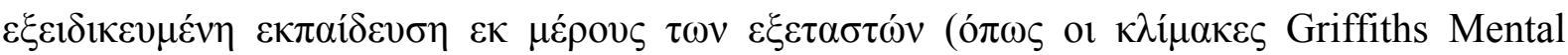

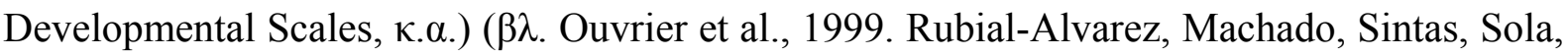
Böhm, \& Peña-Casanova, 2007).

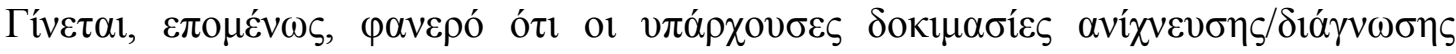

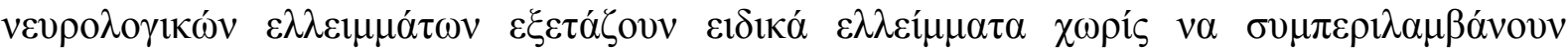

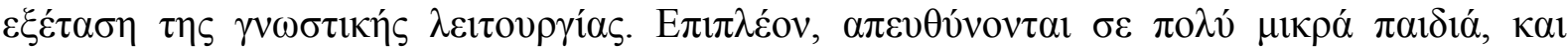

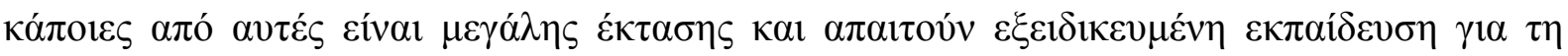

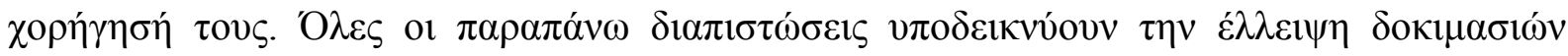

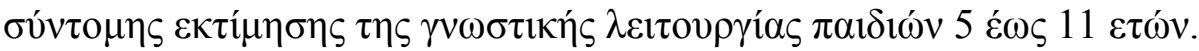

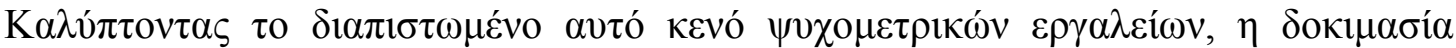

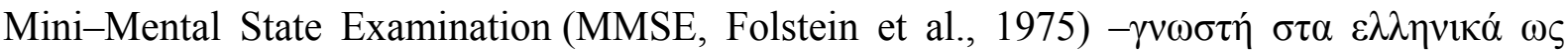

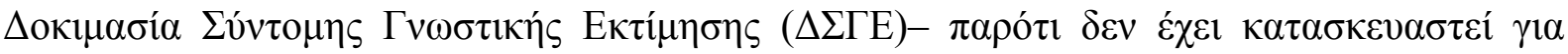

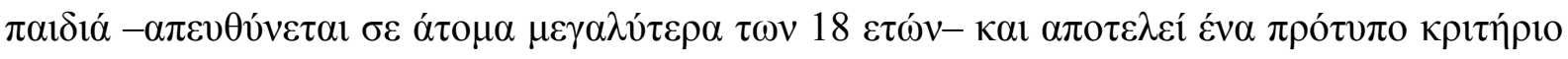

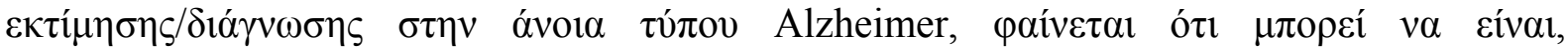

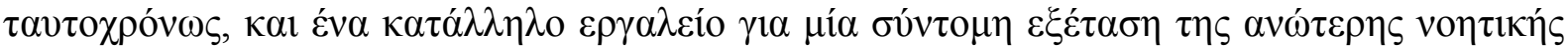

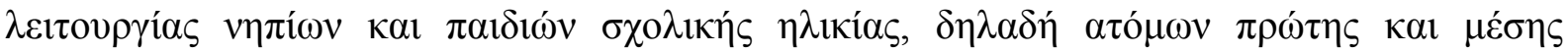

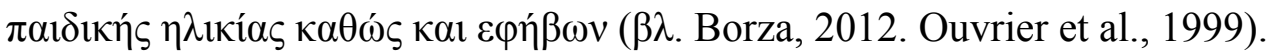

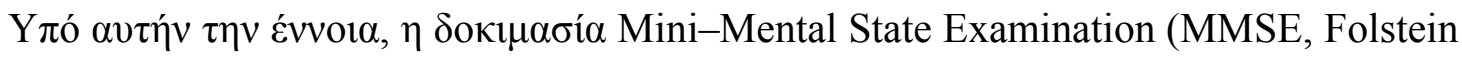

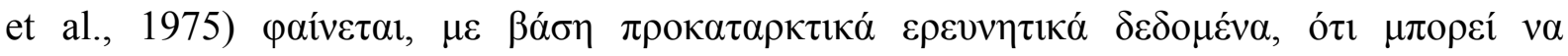

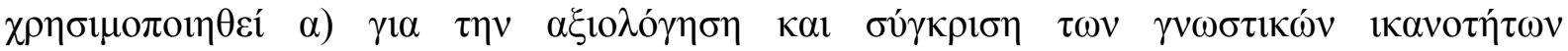

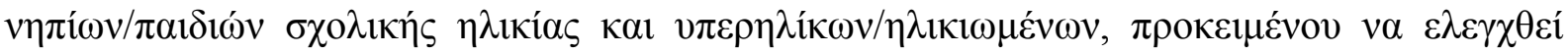

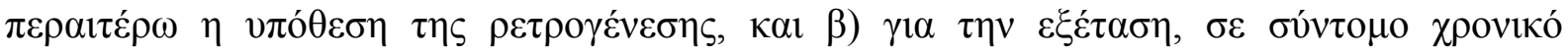

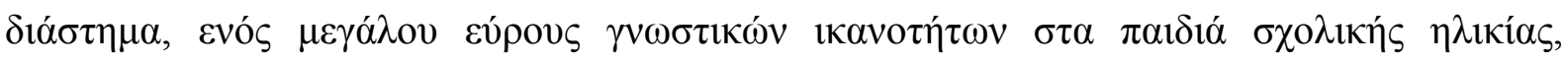

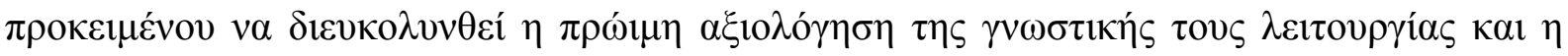




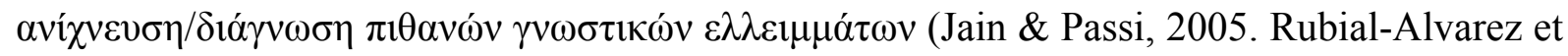
al., 2007. Santos, Pimentel, Rosa, Muzzolon, Antoniuk, \& Bruck, 2012).

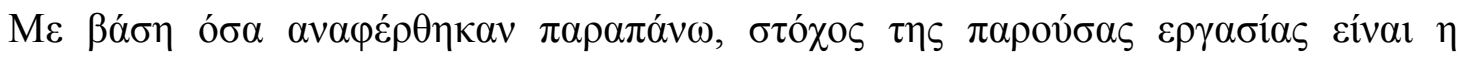

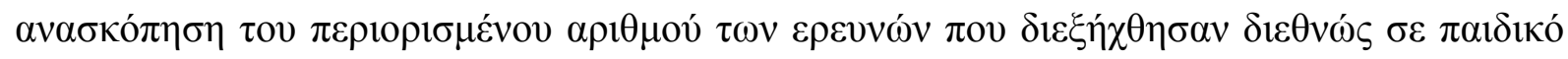

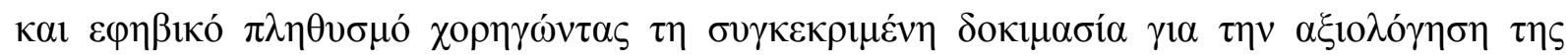

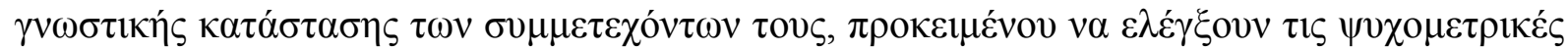

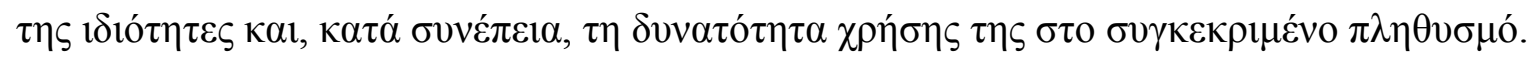

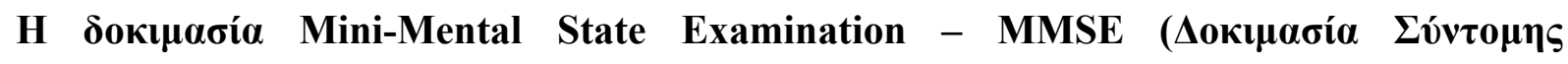

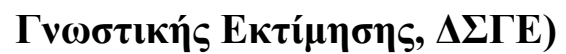

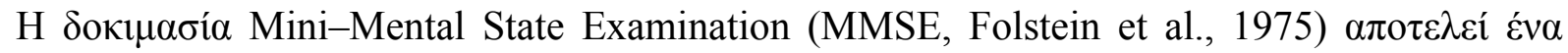

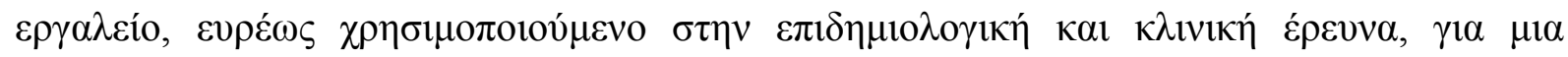

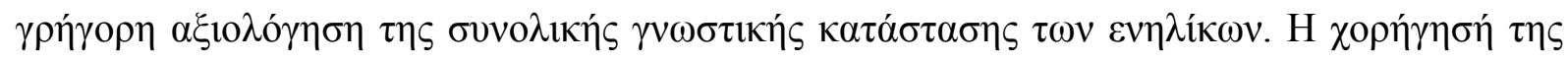

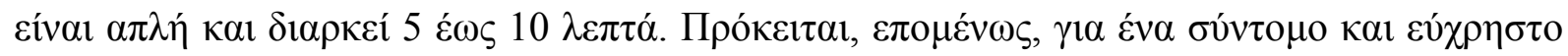

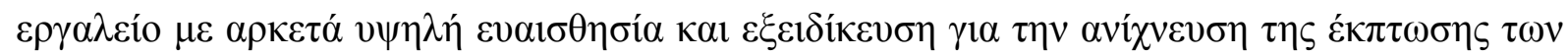

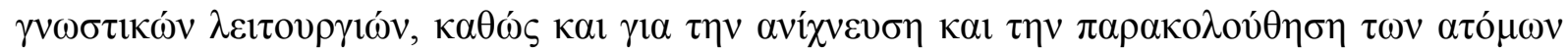

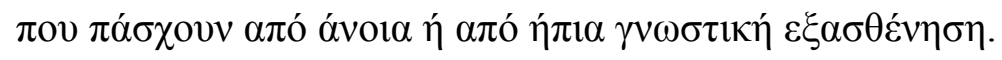

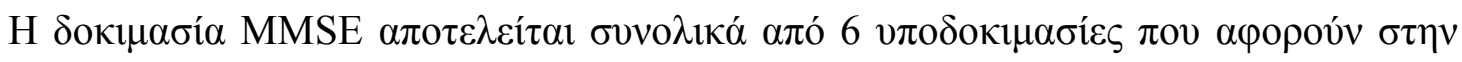

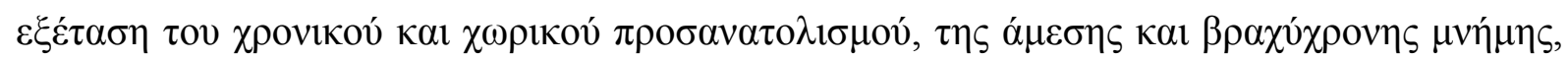

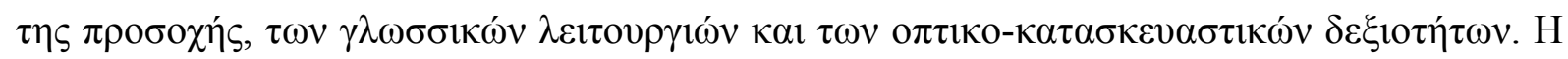

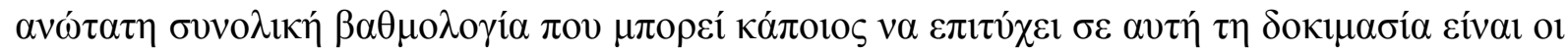

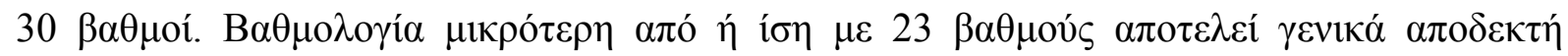

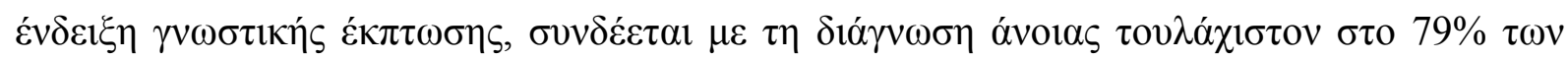

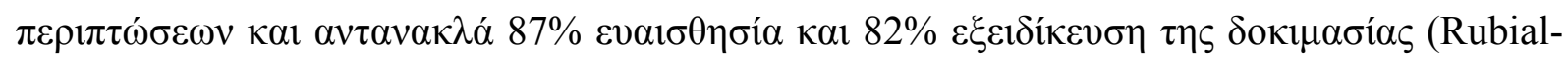




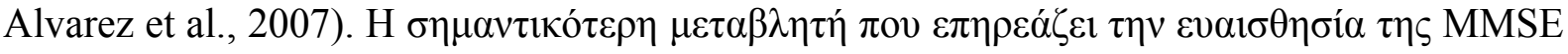

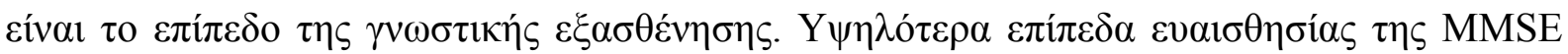

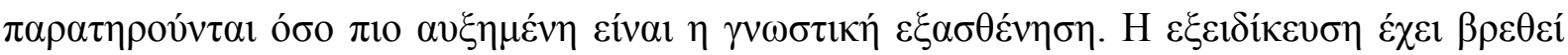

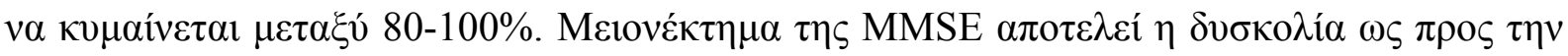

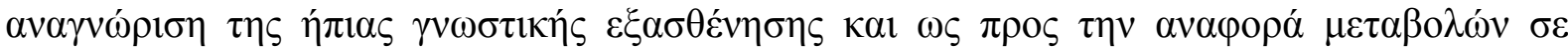

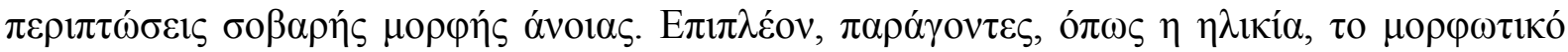

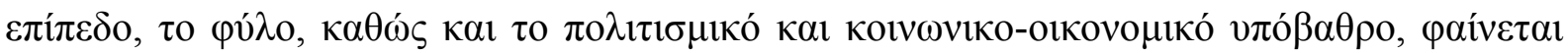

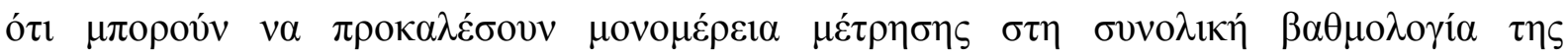

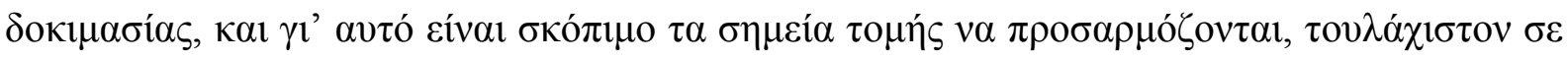

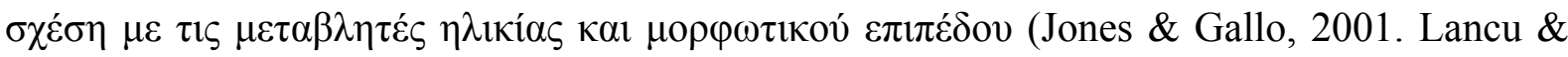

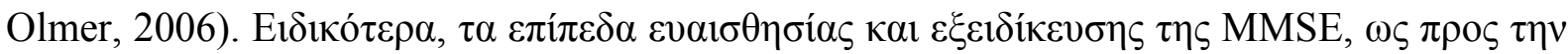

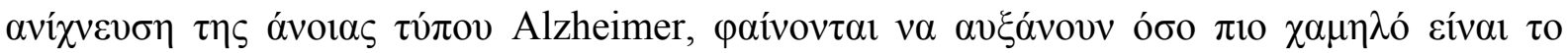

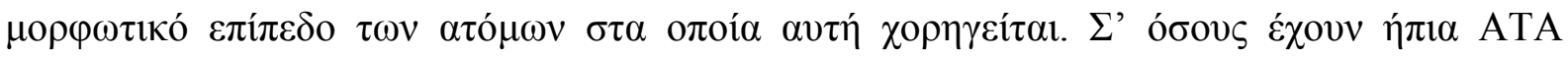

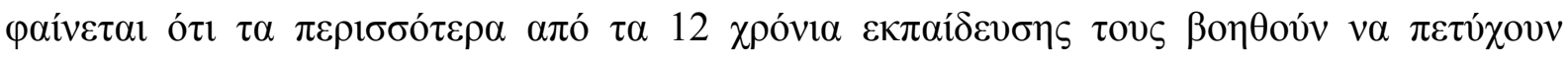

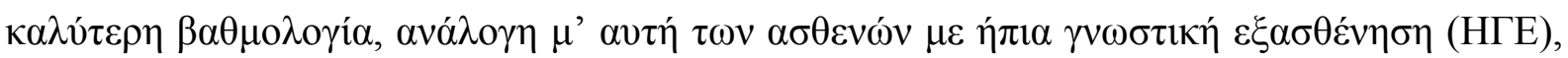

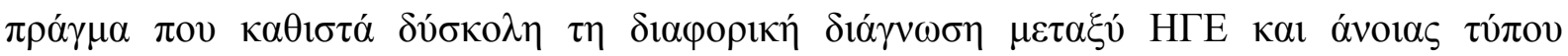

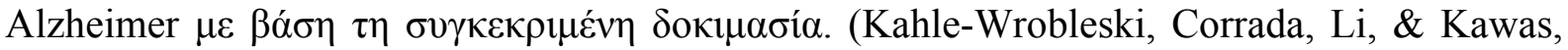

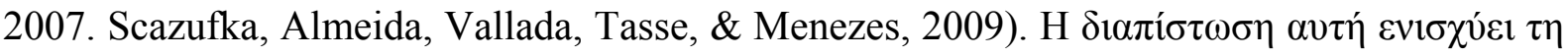

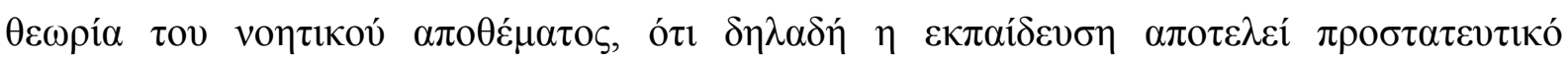

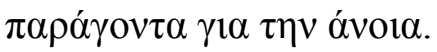

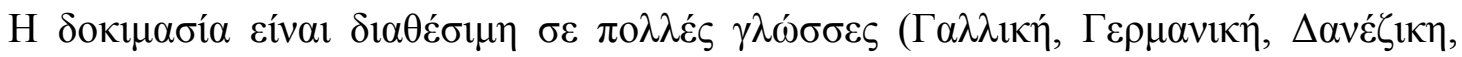

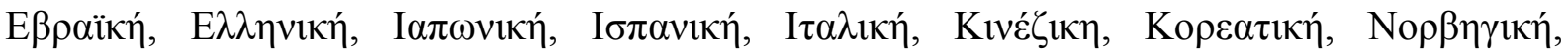

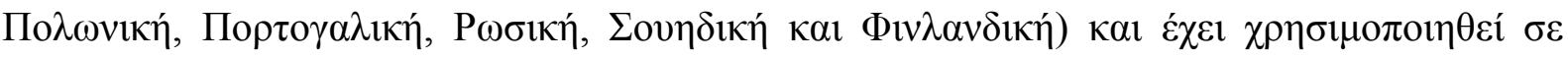

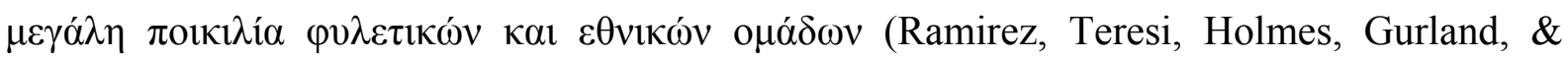

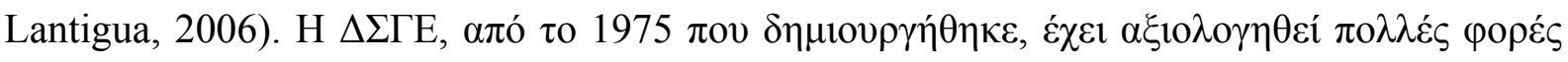




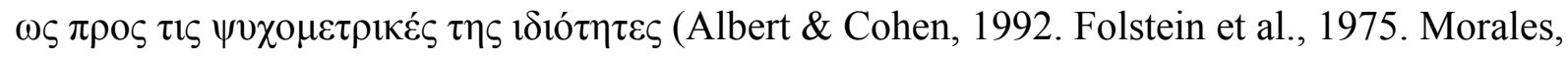
Flowers, Gutierrez, Kleinman, \& Tenesi, 2006. Shigemori, Ohgi, Okuyama, Shimura, \&

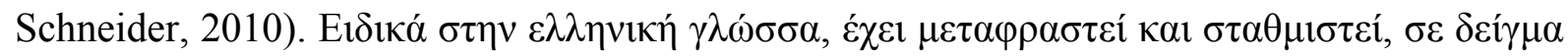

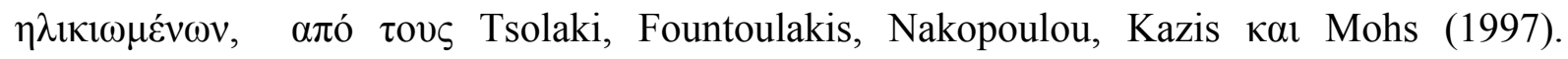

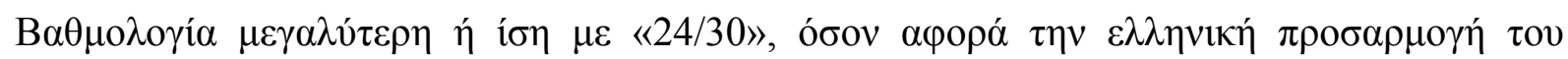

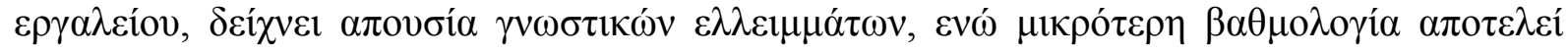
$\dot{\varepsilon ́ v \delta \varepsilon 1 \xi \eta \gamma \nu \omega \sigma \tau \iota \kappa \eta ́ \varsigma ~ \varepsilon ́ \kappa \pi \tau \omega \sigma \eta \varsigma ~(T s o l a k i ~ e t ~ a l ., ~ 1997) . ~}$

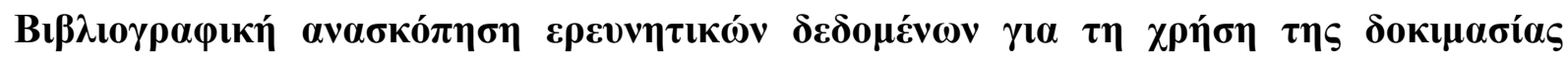

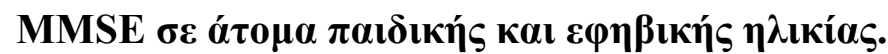

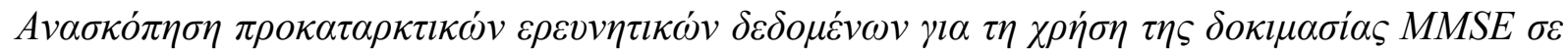

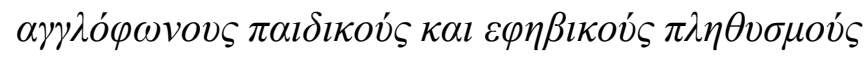

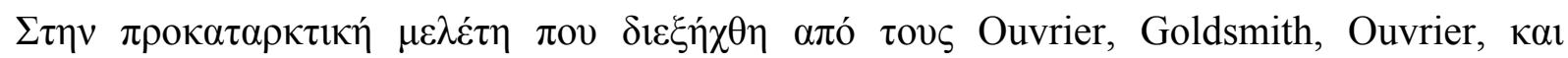

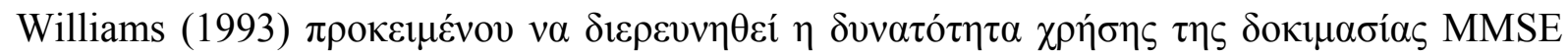

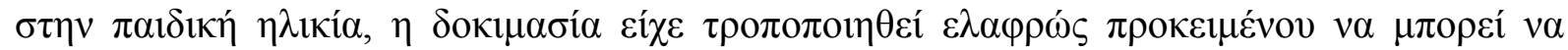

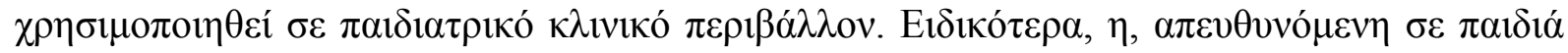

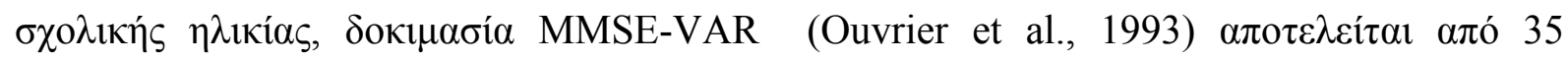

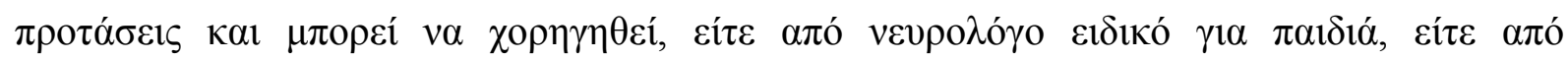

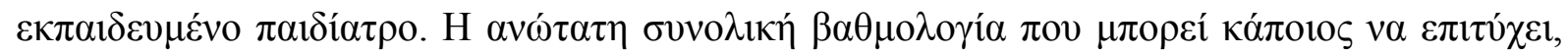

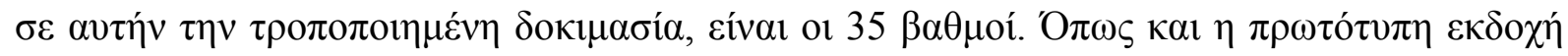

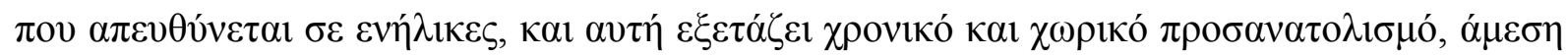

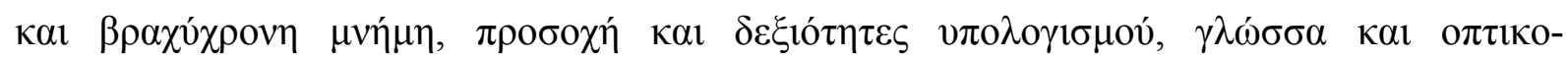

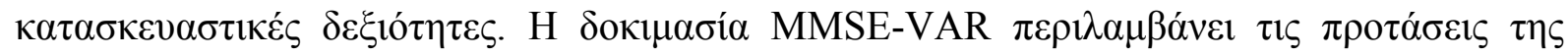




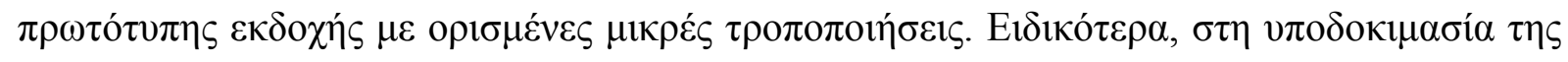

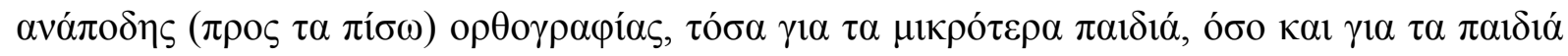

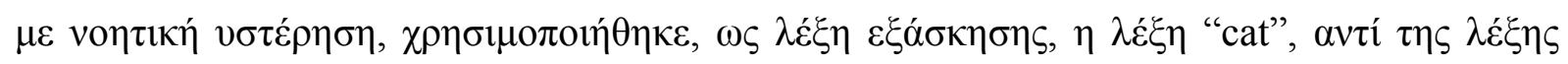

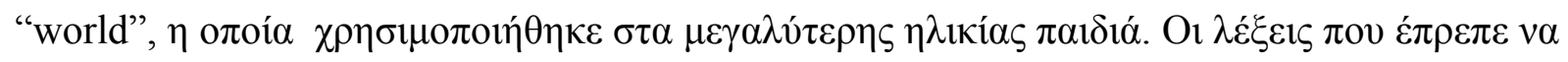

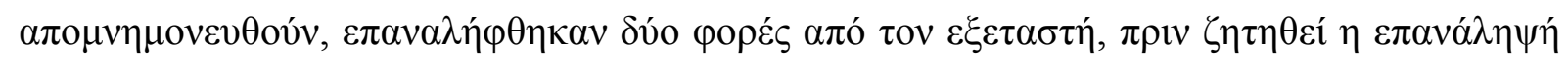

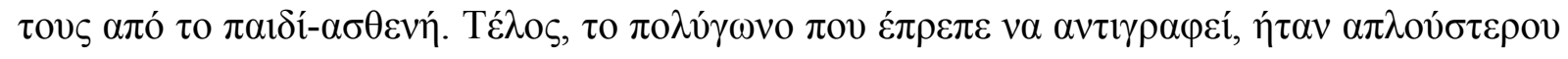

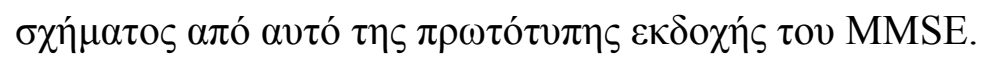

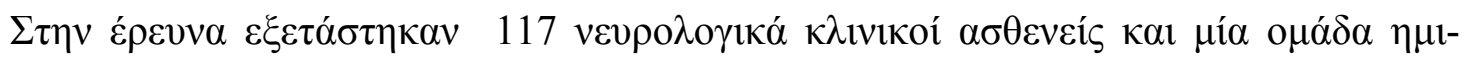

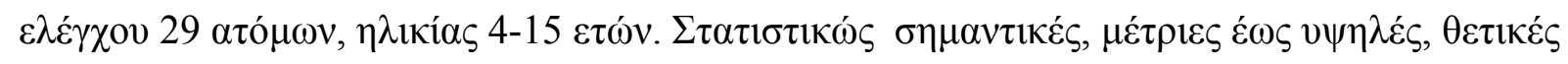

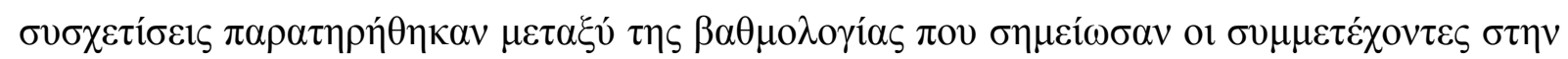

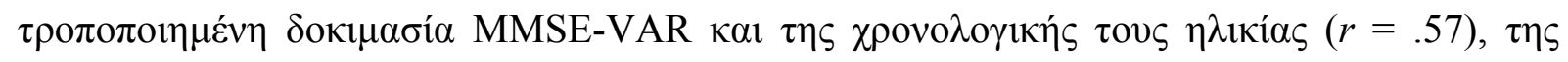

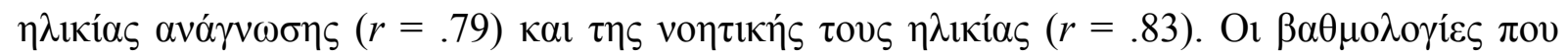

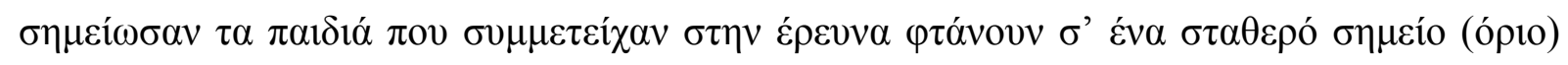

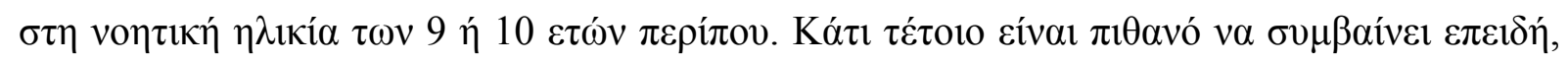

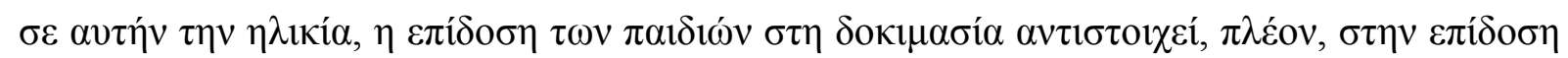

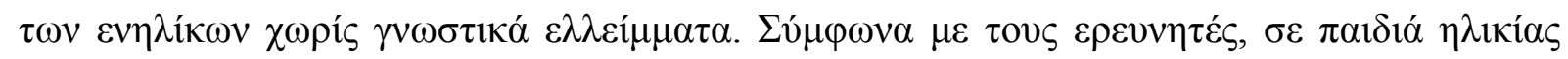

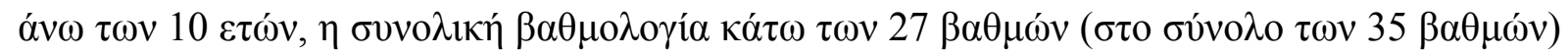

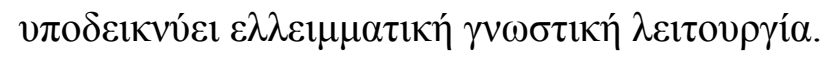

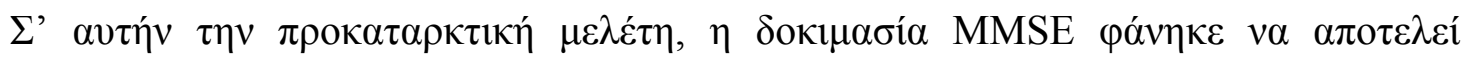

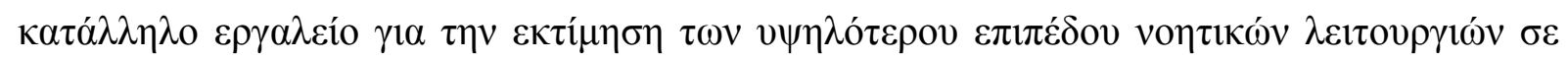

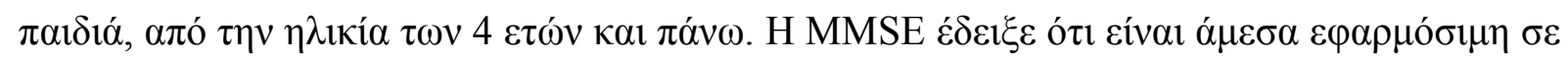

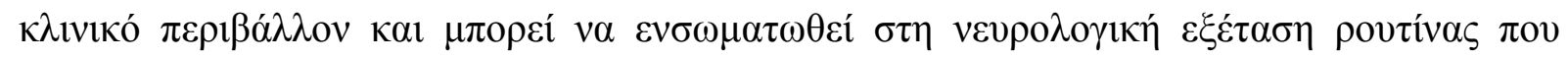

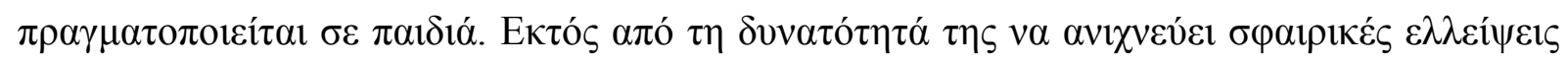

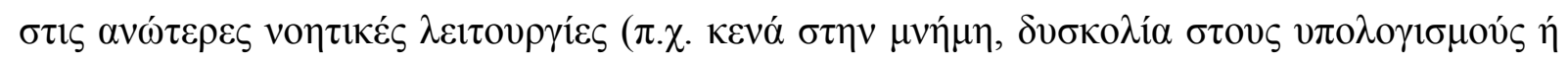

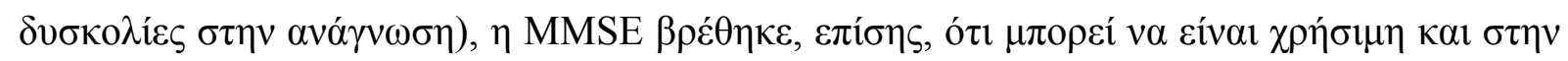




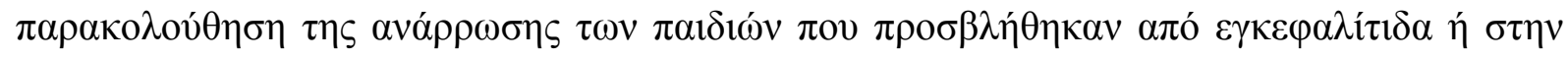

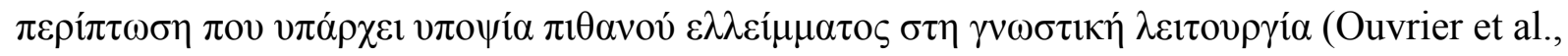
1993).

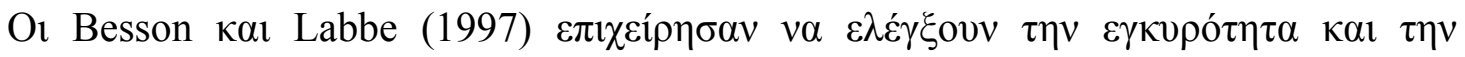

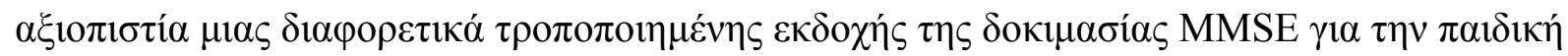

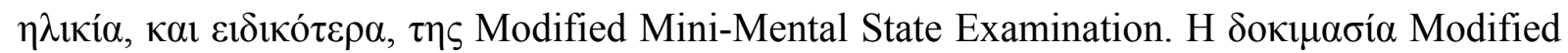

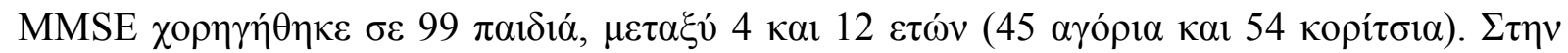

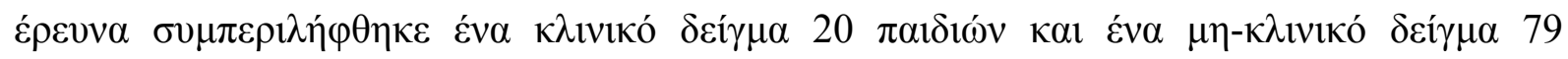

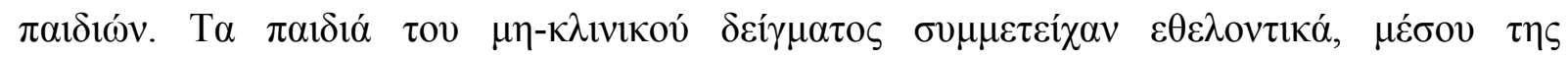

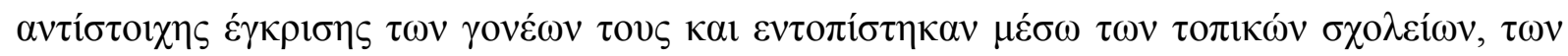

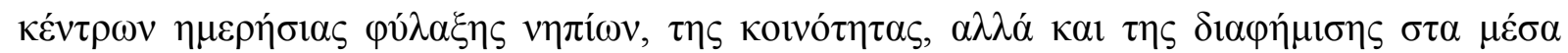

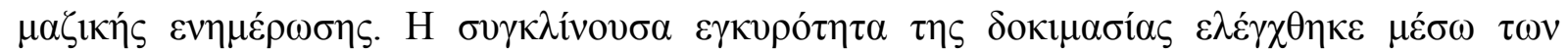

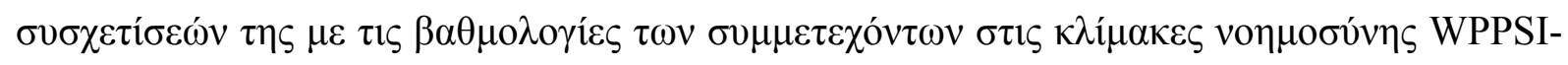

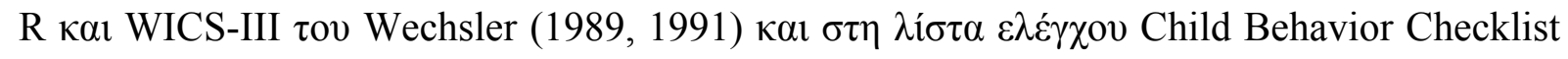

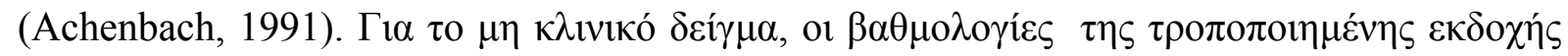

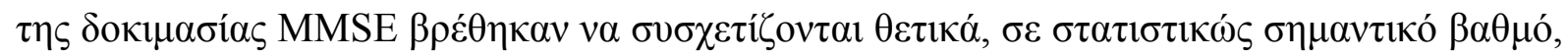

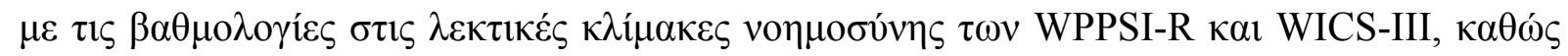

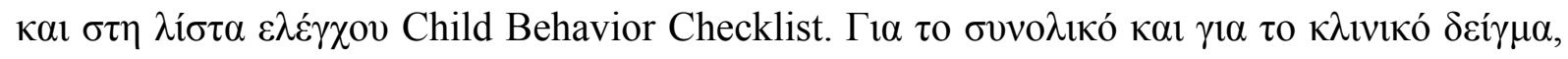

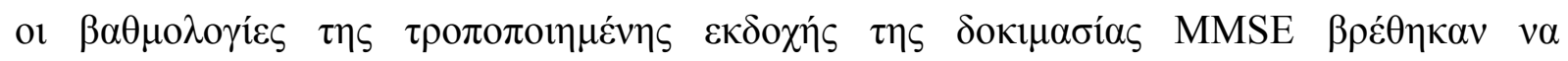

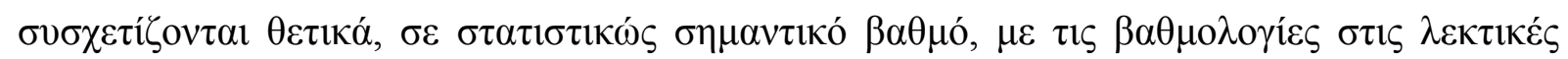

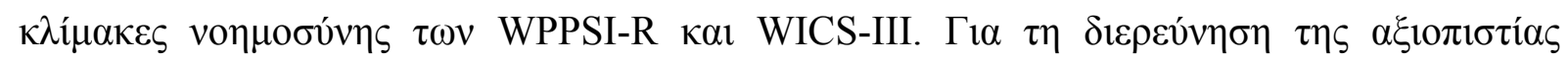

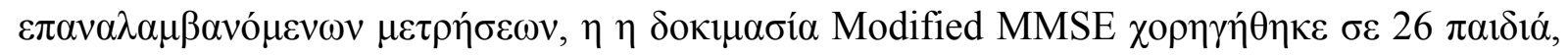

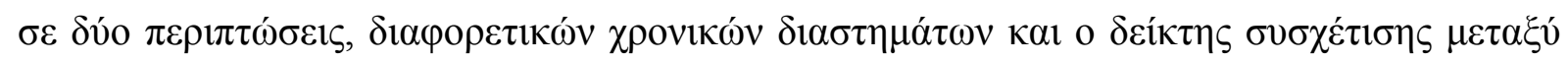

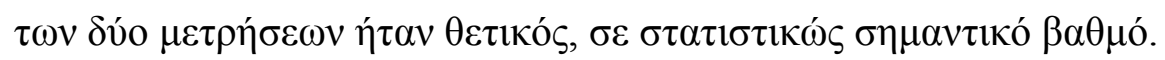




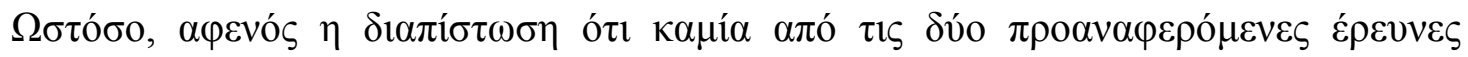

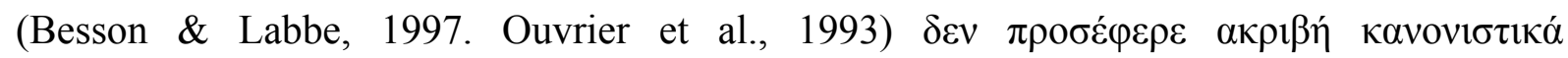

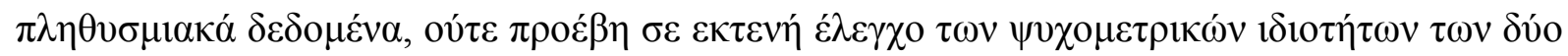

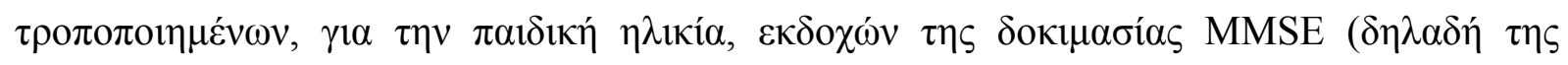

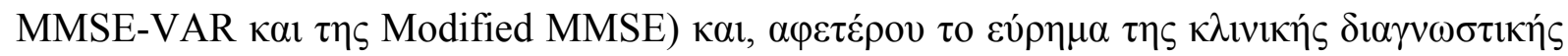

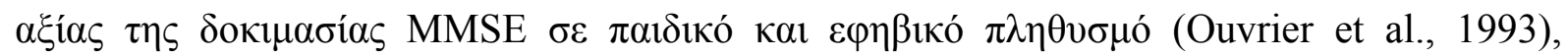

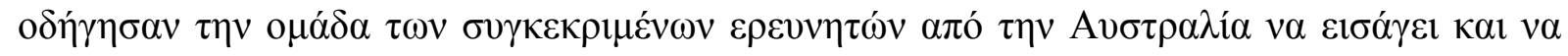

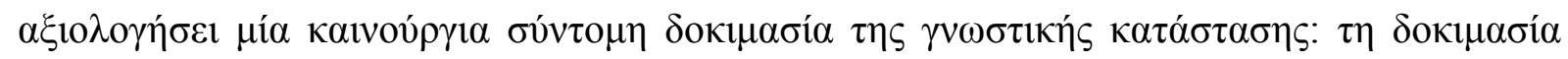
School-Years Screening Test for Evaluation of Mental Status (SYSTEMS) (Ouvrier et al.,

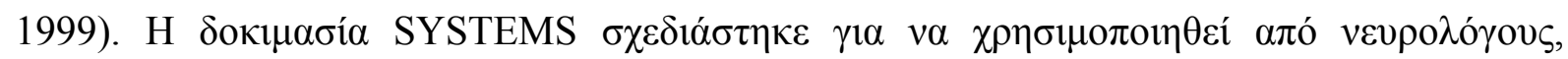

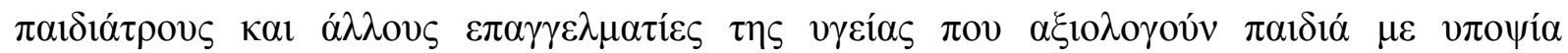

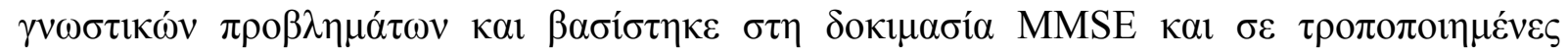

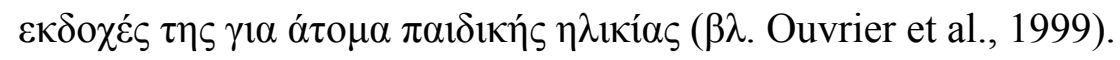

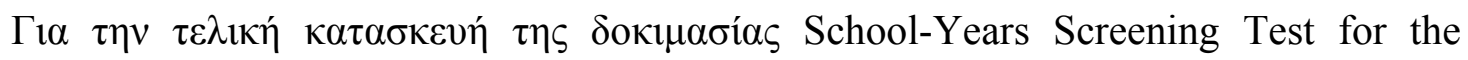

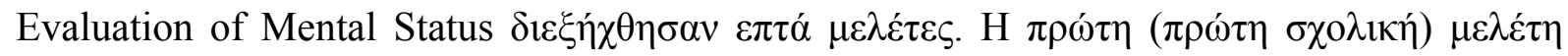

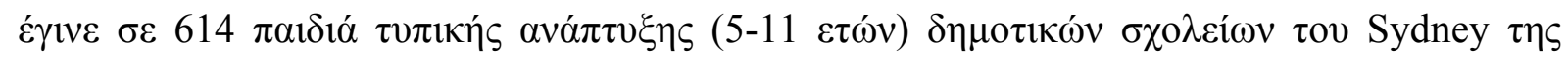

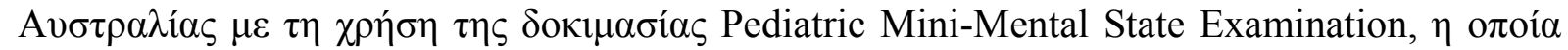

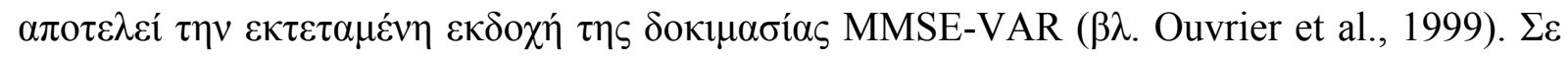

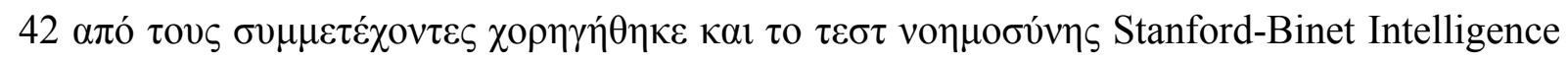

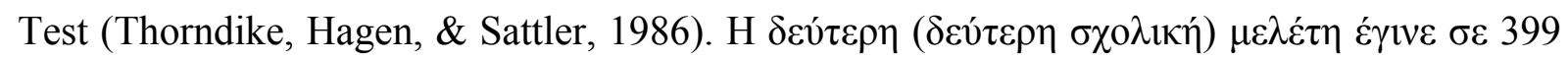

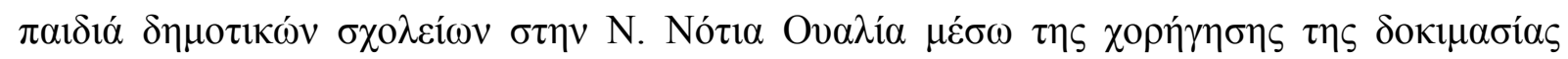

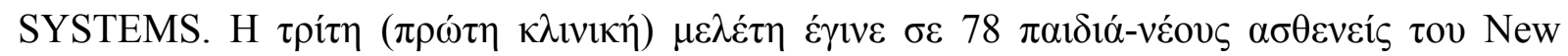

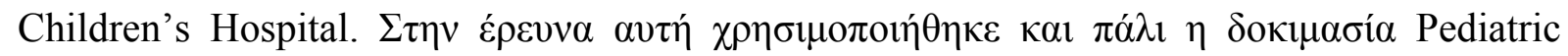

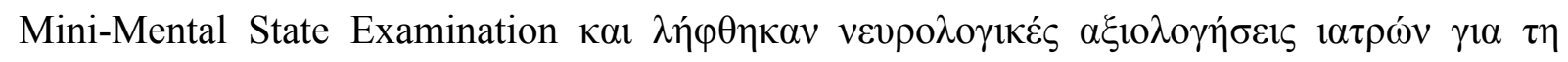

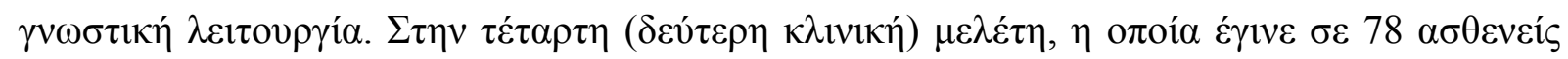




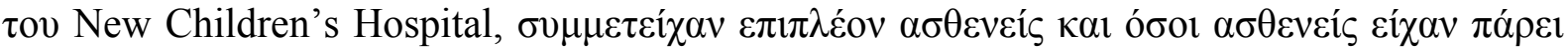

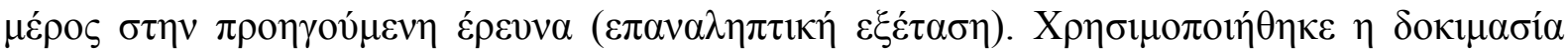

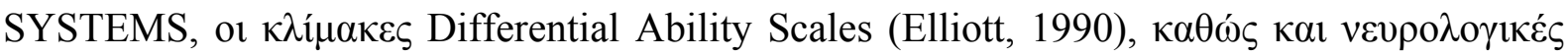

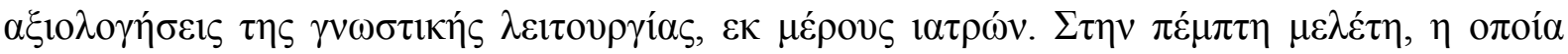

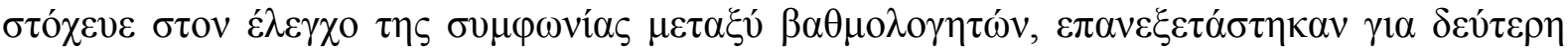

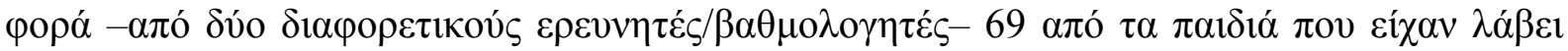

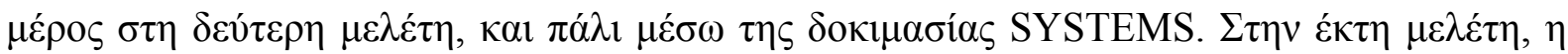

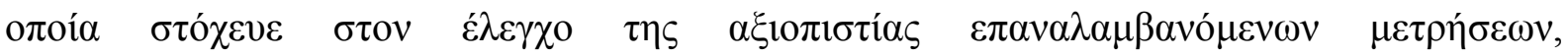

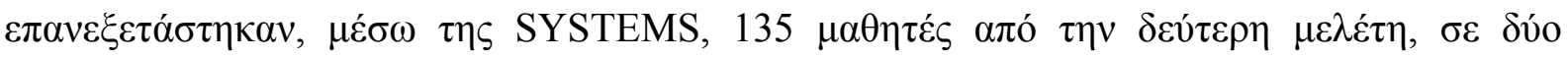

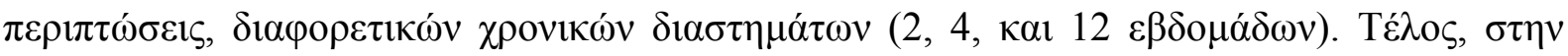

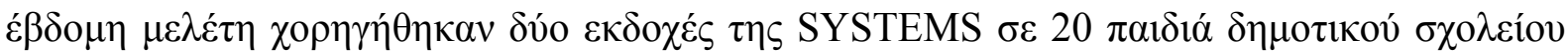

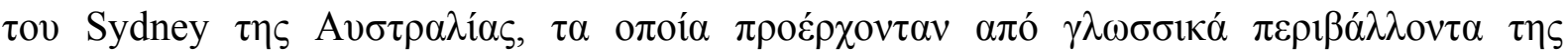

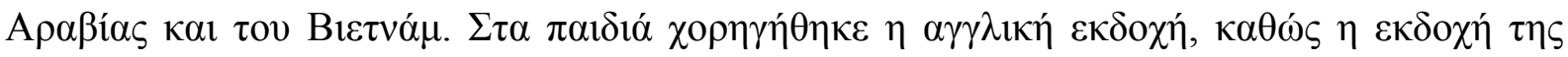

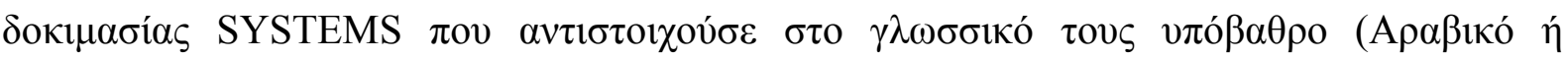

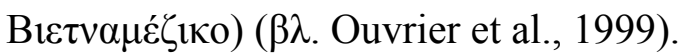

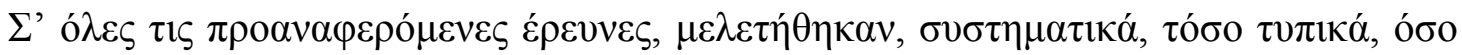

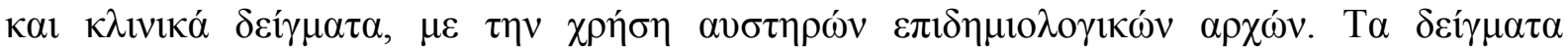

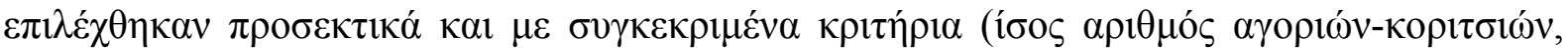

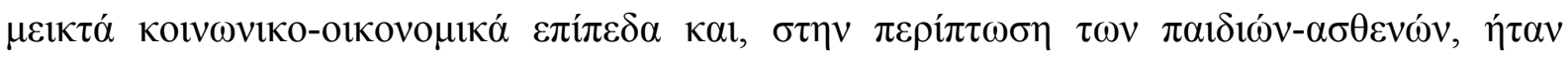

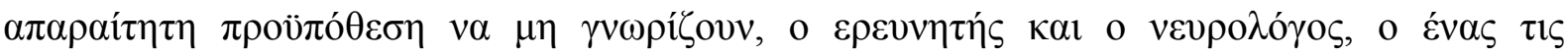

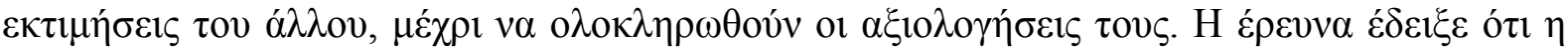

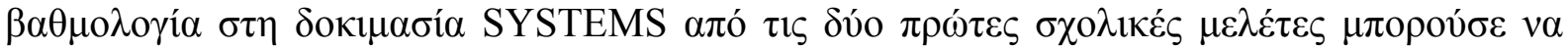

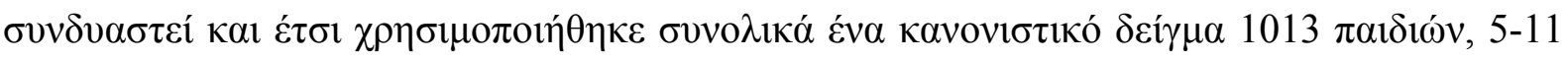

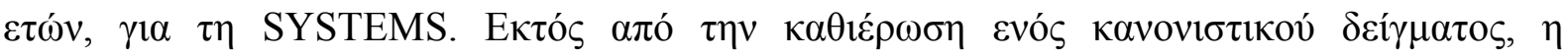

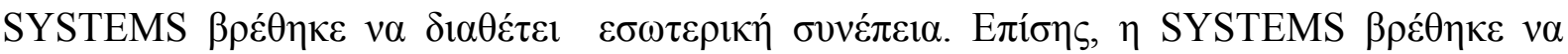




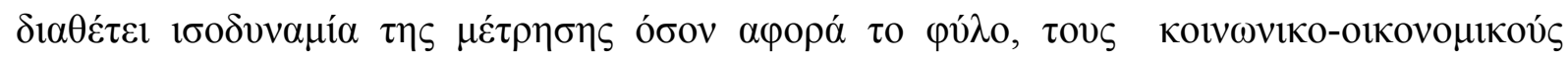

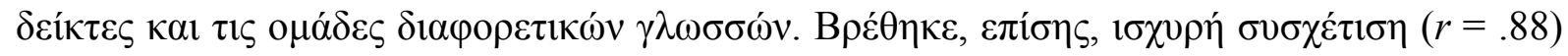

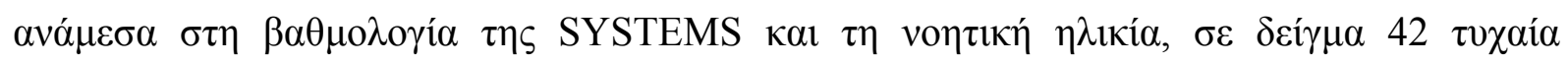

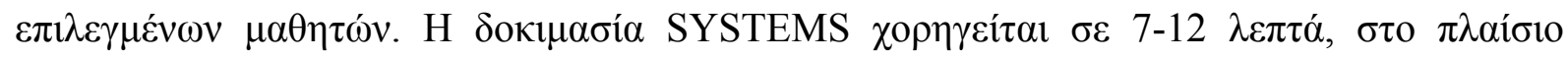

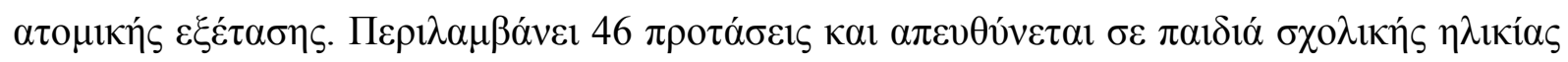

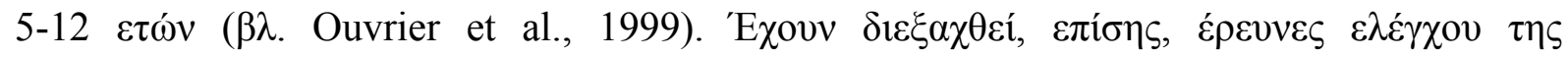

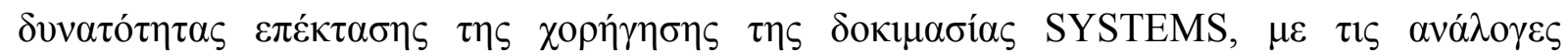

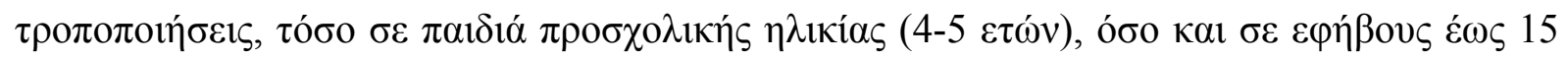

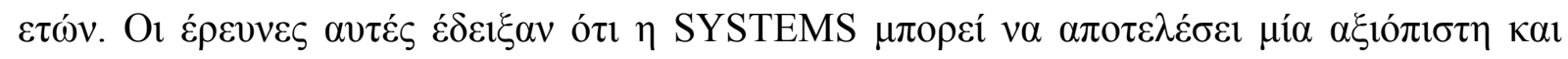

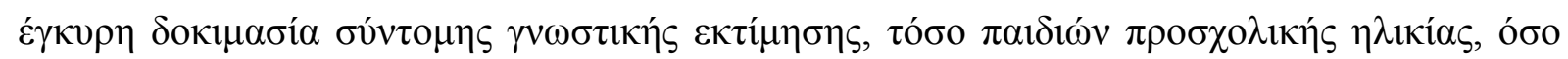

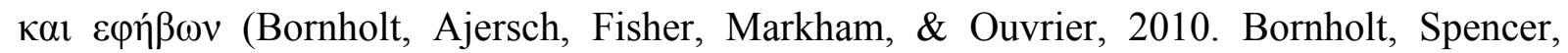

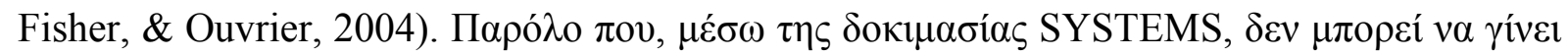

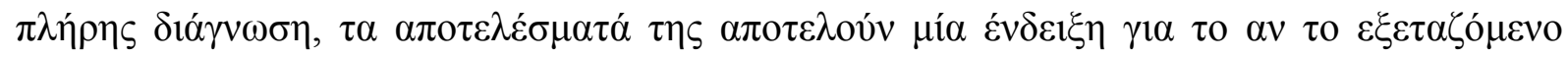

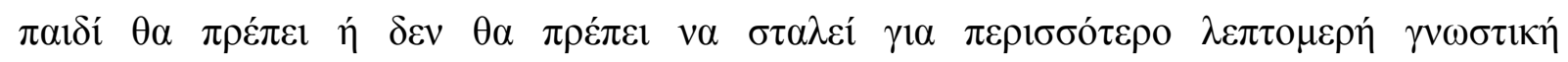

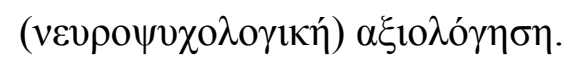

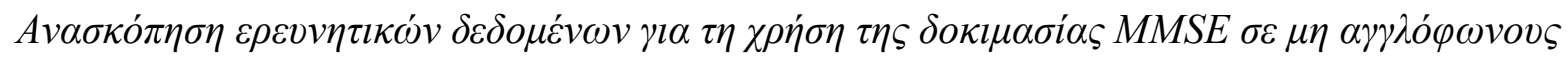
$\pi \alpha \imath \delta \imath \kappa o v ́ \varsigma \kappa \alpha l \varepsilon \varphi \eta \beta \imath \kappa o v ́ \varsigma \pi \lambda \eta \theta v \sigma \mu o v ́ \varsigma$

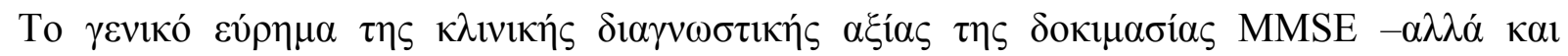

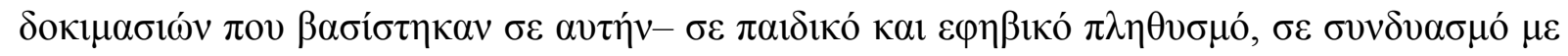

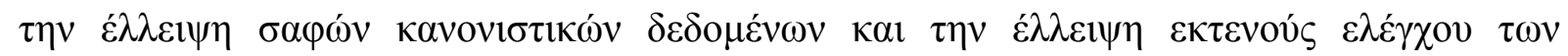

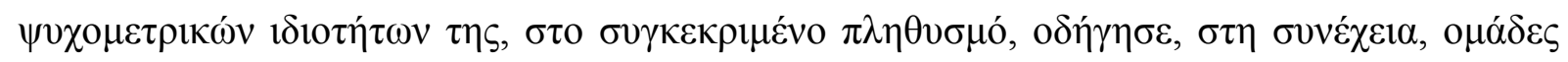

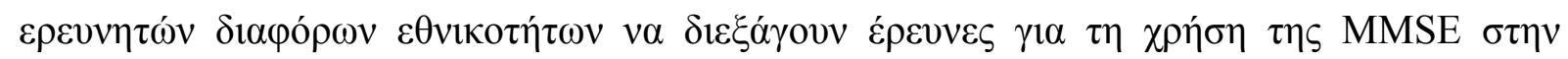




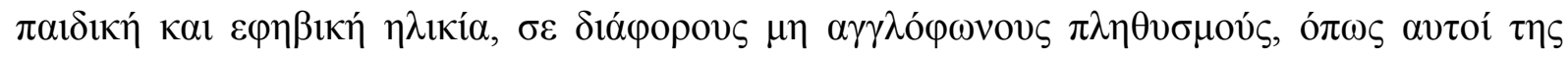

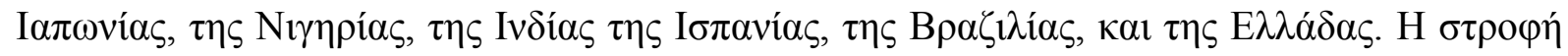

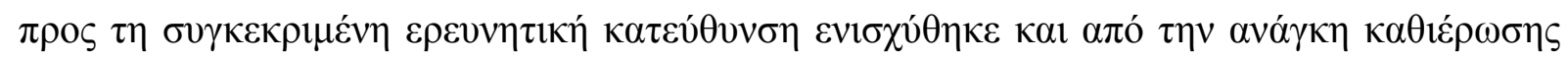

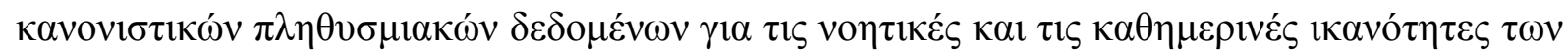

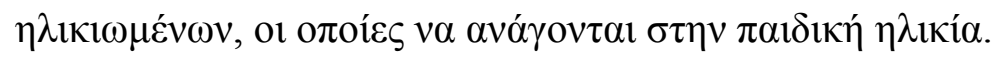

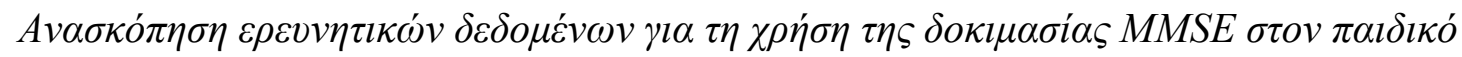

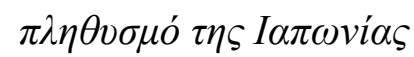

Oı Shoji, Fukushima, Wakayama, Shizuka-Ikeda, Ikeda, Kawakami et al. (2002) $\delta 1 \varepsilon \xi \eta ́ \gamma \alpha \gamma \alpha \nu$

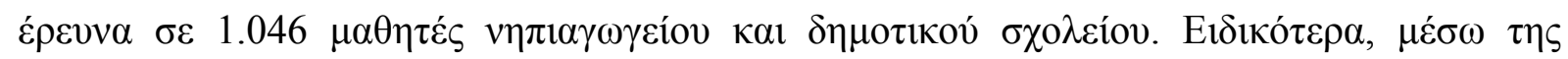

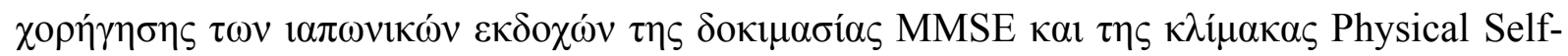

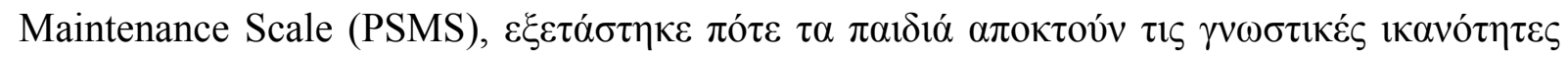

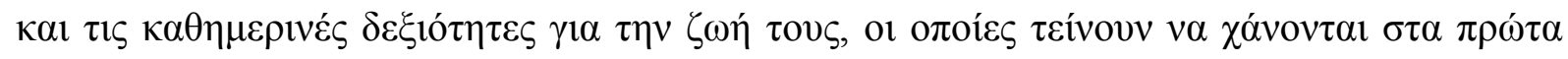

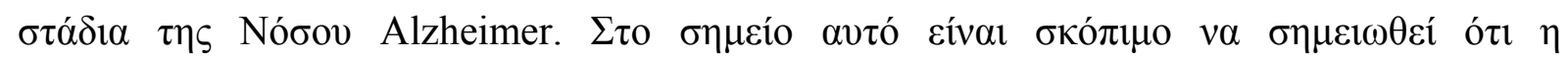

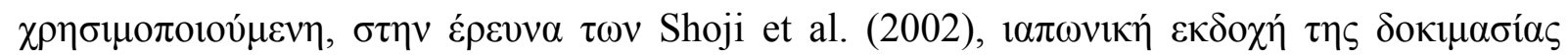

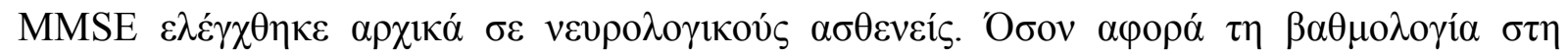

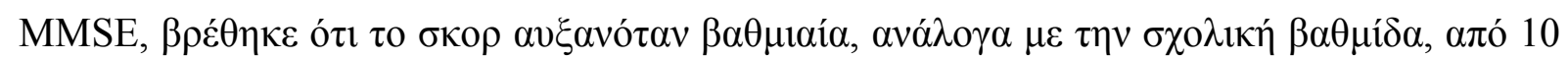

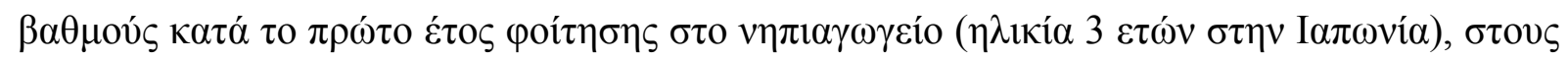

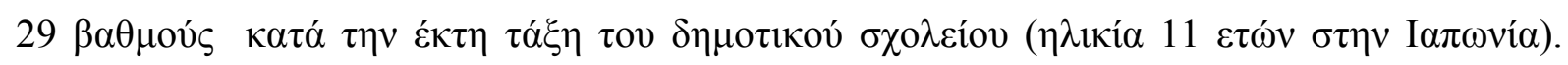

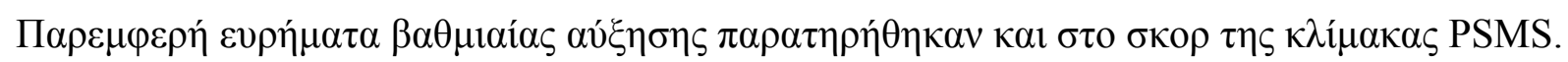

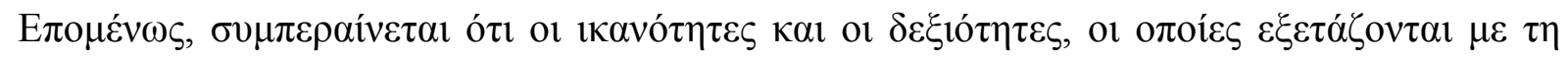

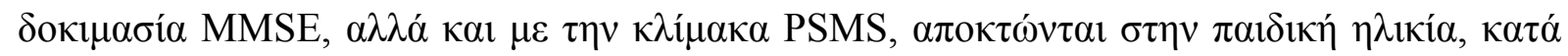

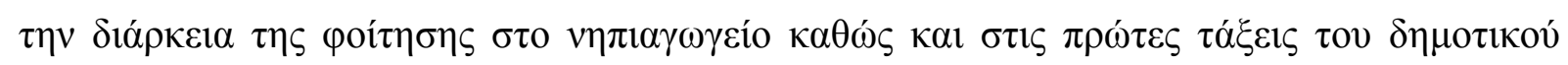

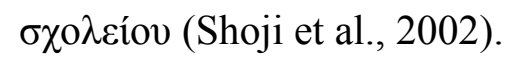




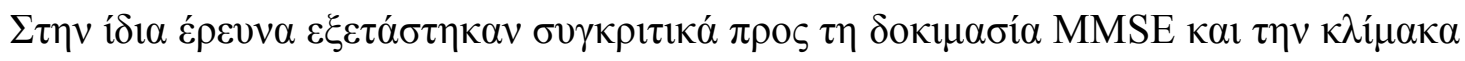

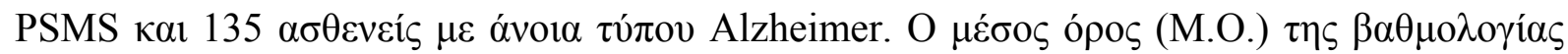

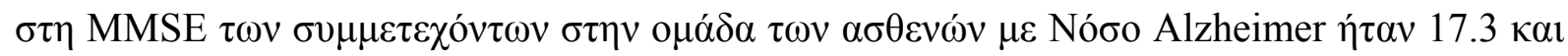

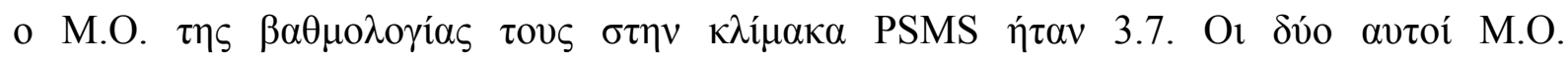

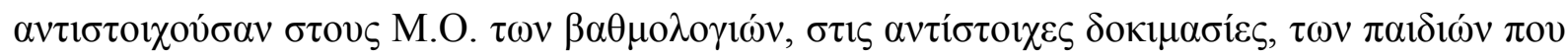

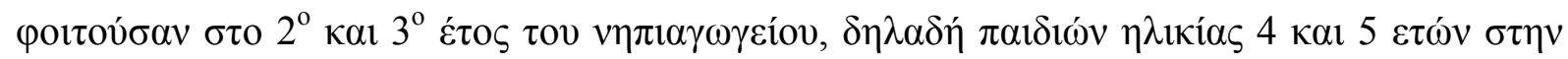

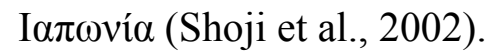

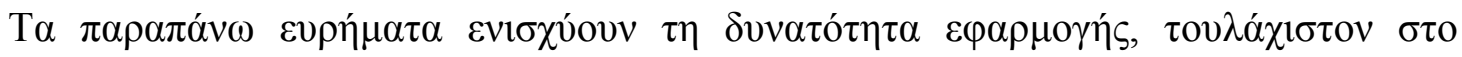

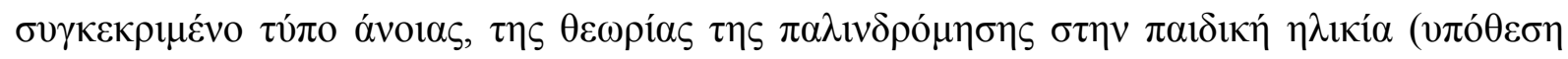

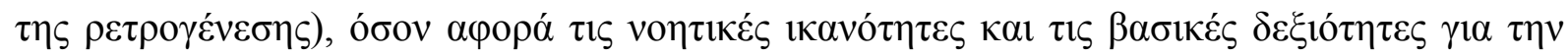

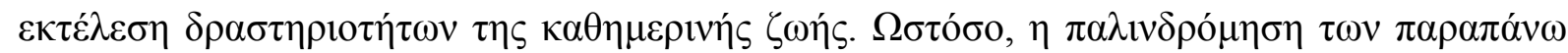

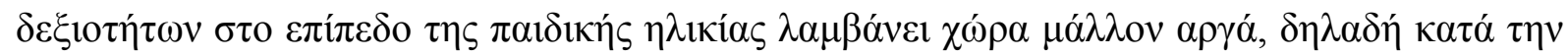

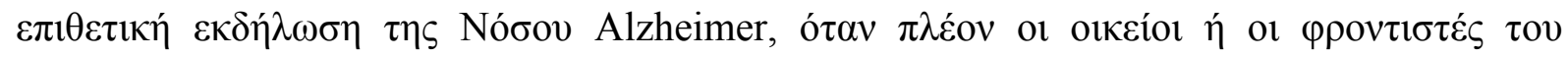

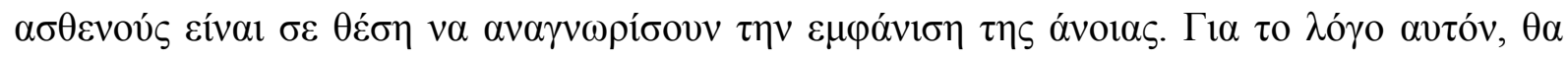

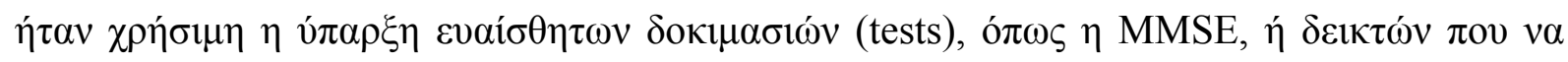

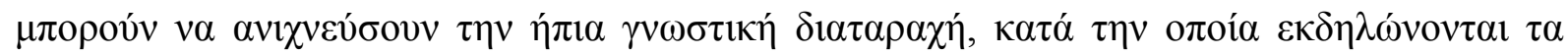

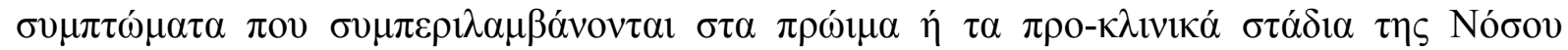

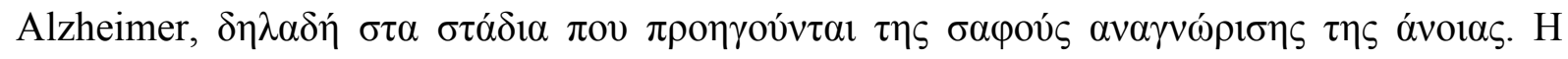

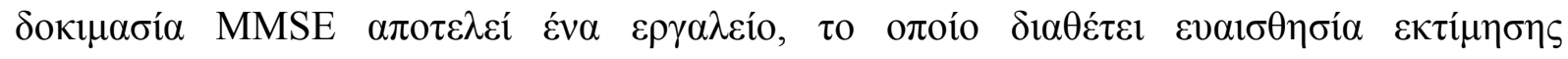

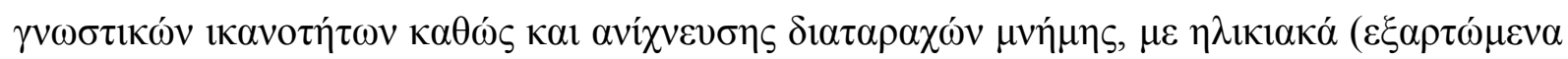

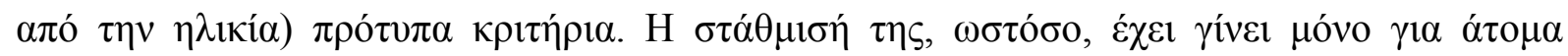

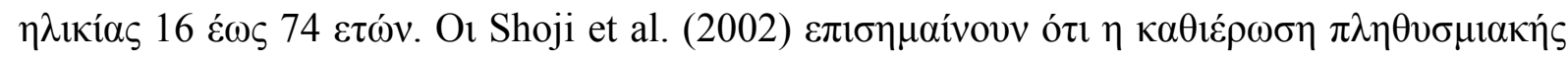

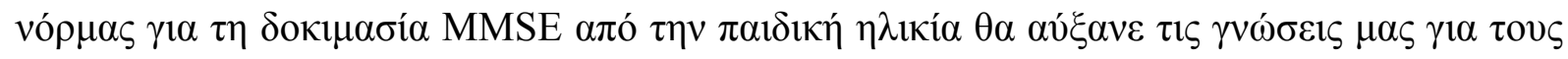

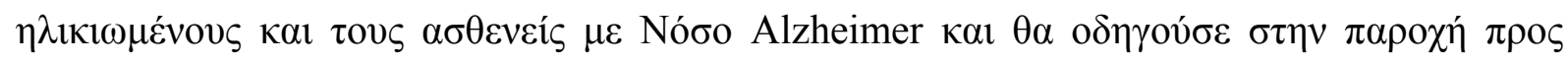

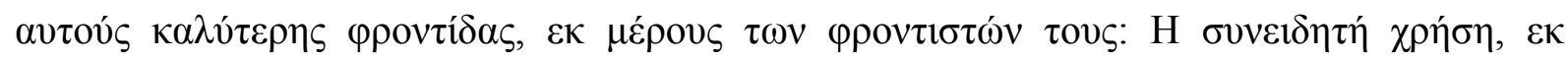




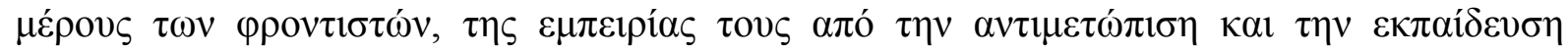

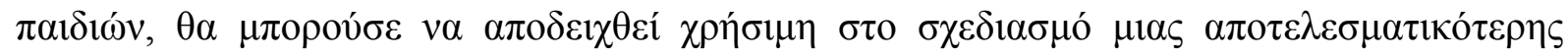

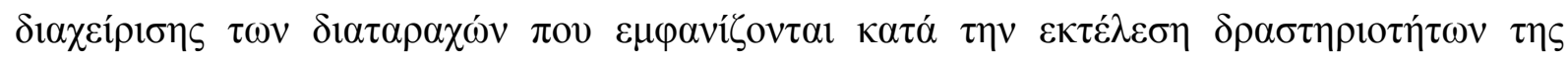

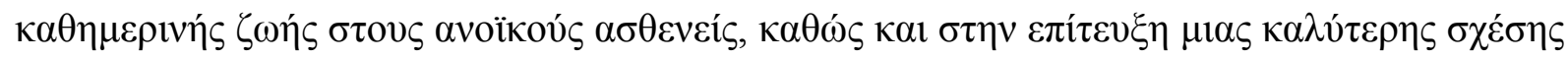

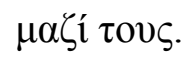

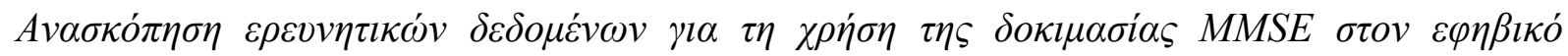

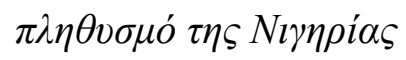

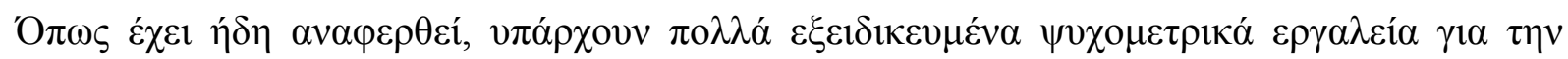

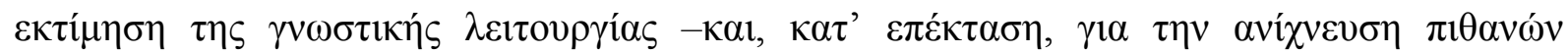

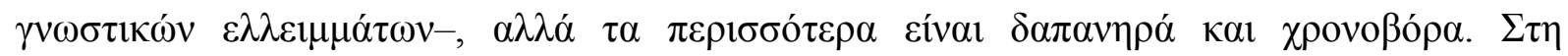

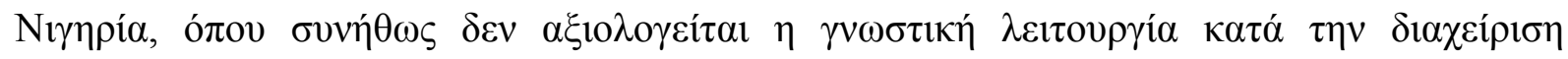

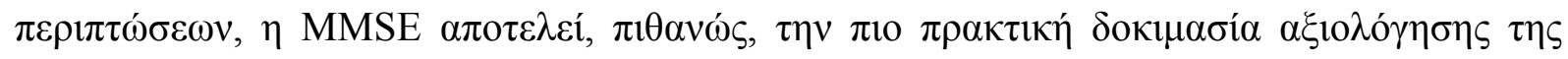

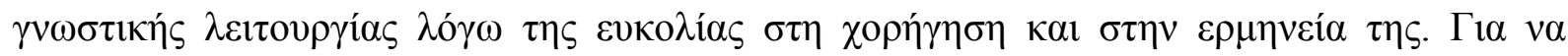

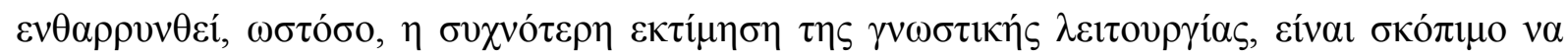

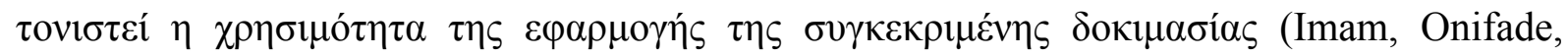
Durodoye, Aje, Sogaolu, Kehinde et al., 2003).

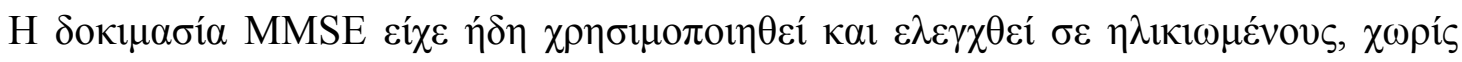

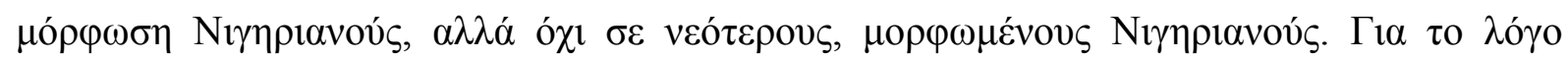

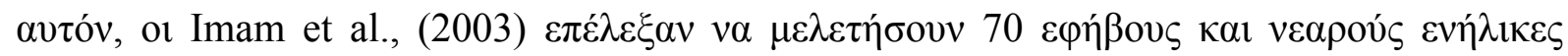

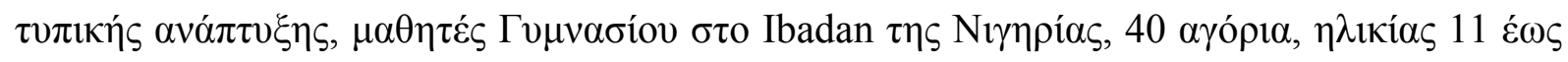

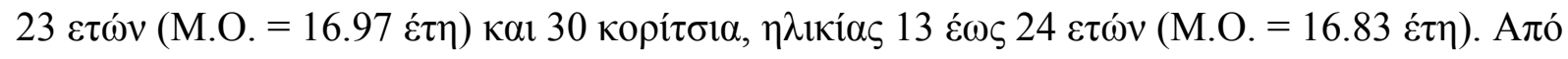

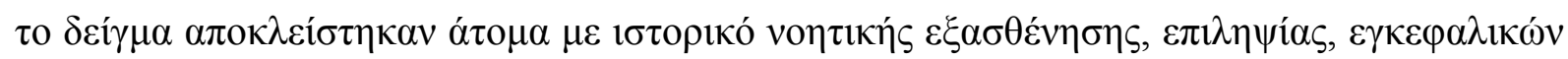




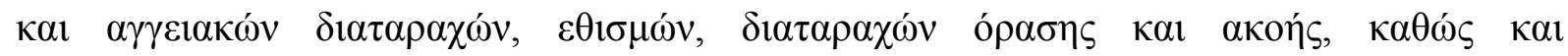

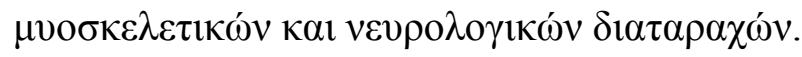

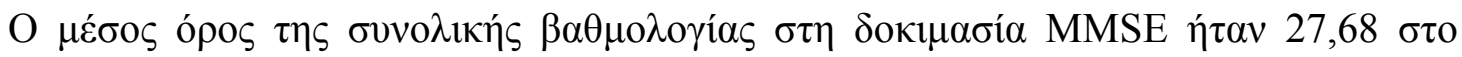

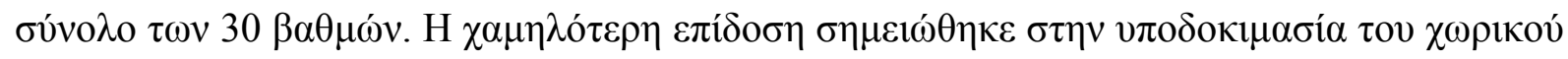

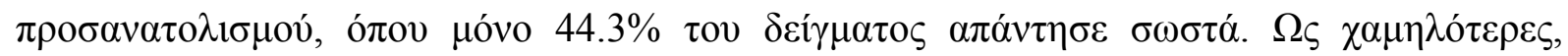

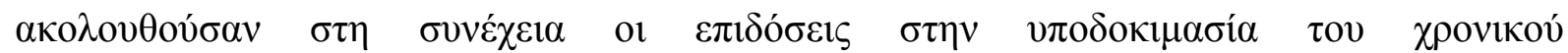

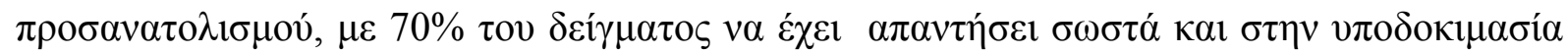

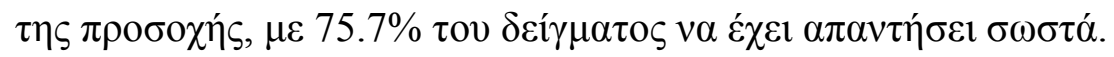

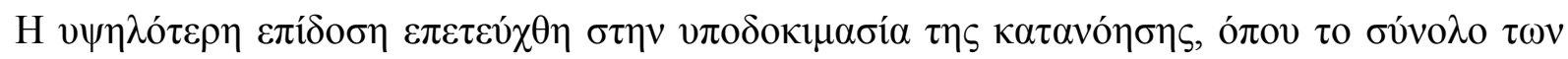

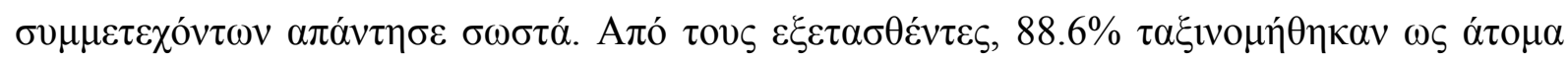

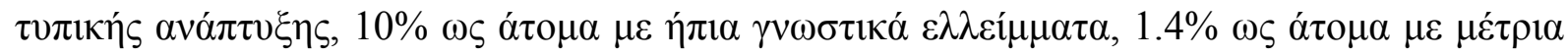

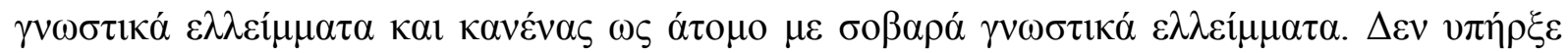

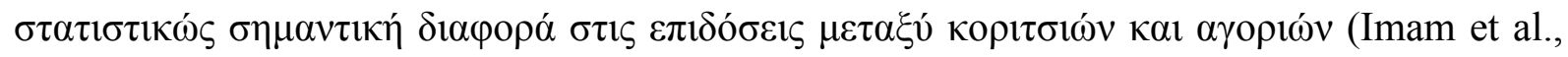
2003).

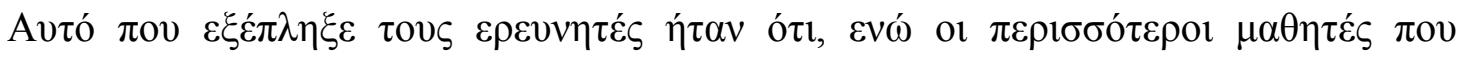

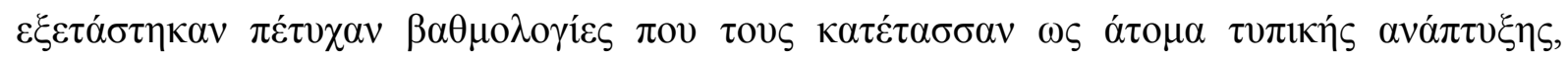

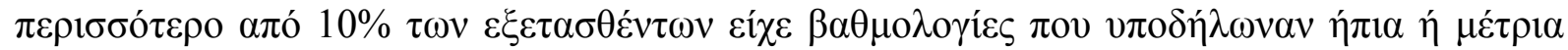

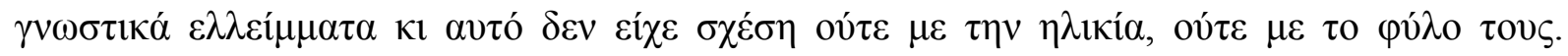

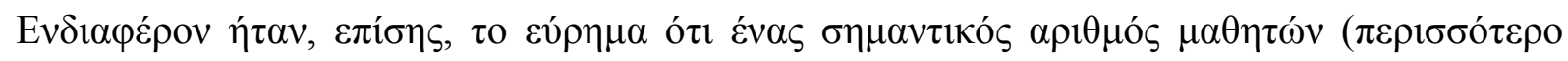

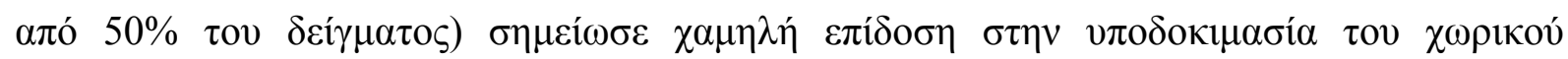

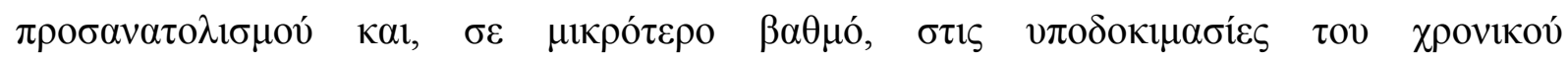

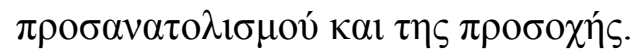

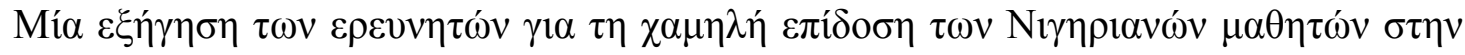

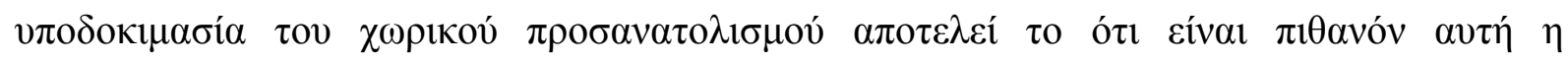

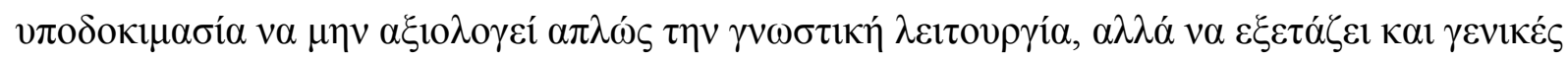




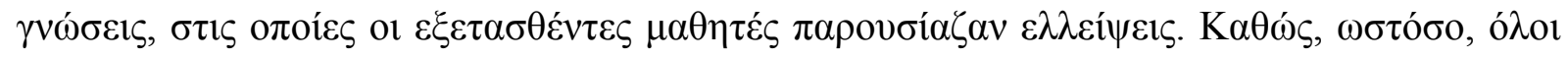

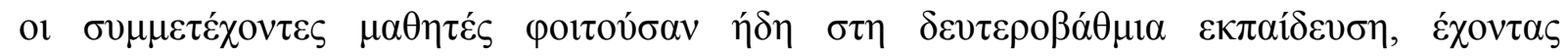

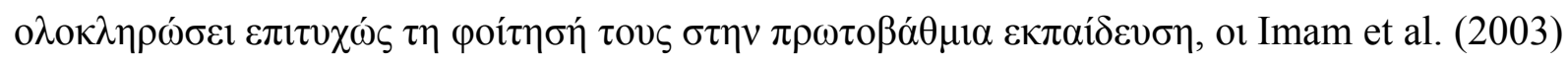

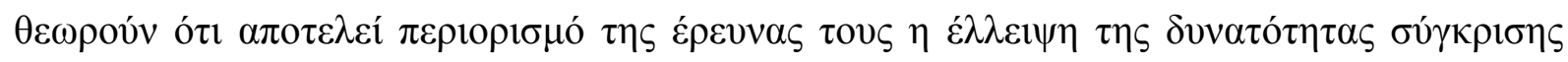

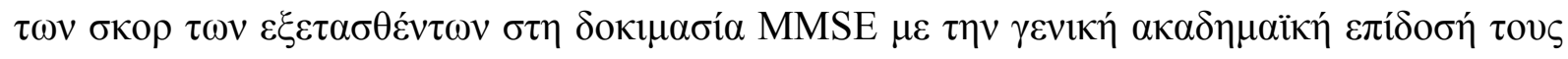

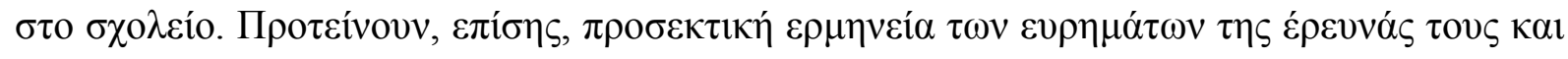

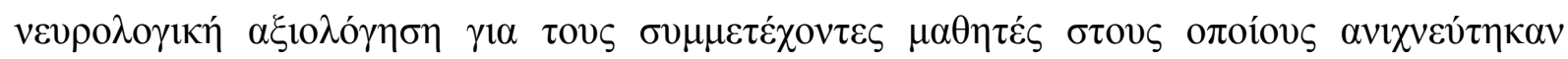

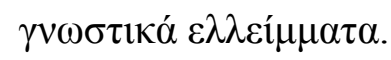

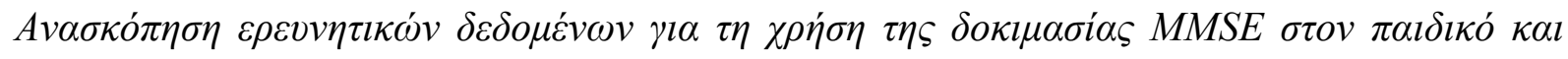
$\varepsilon \varphi \eta \beta \imath \kappa o ́ ~ \pi \lambda \eta \theta v \sigma \mu o ́ ~ \tau \eta \varsigma I v \delta i ́ \alpha \varsigma$

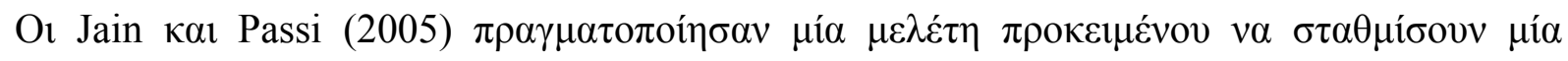

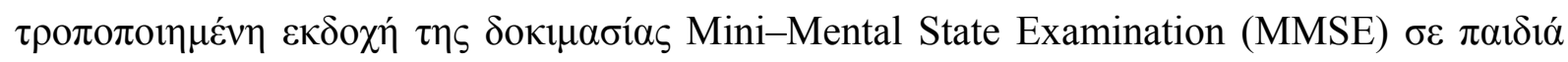

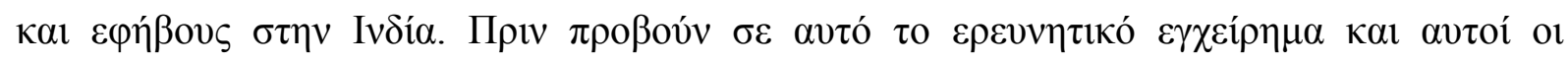

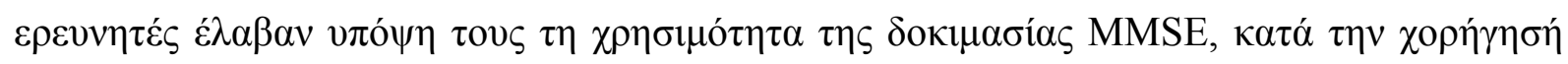

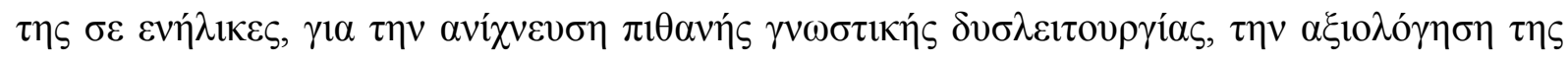

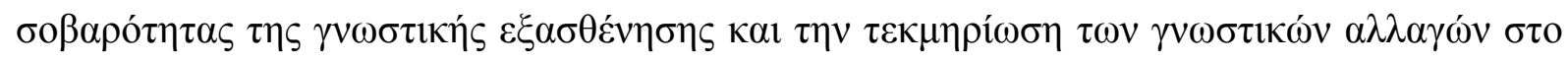

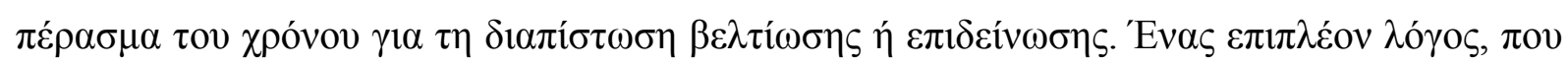

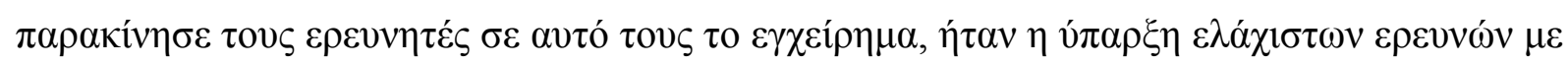

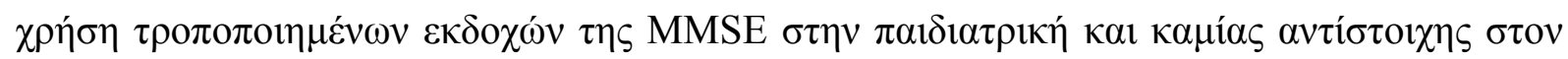

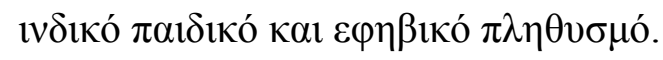

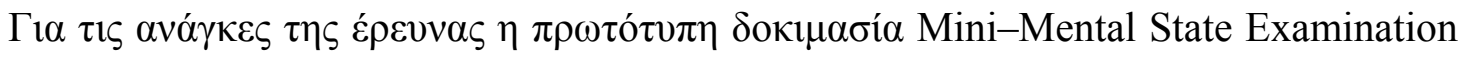

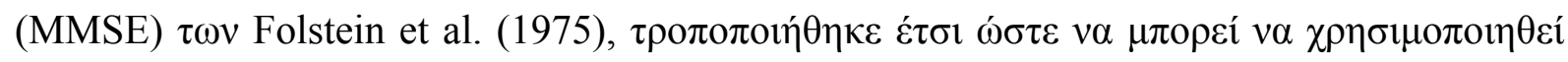

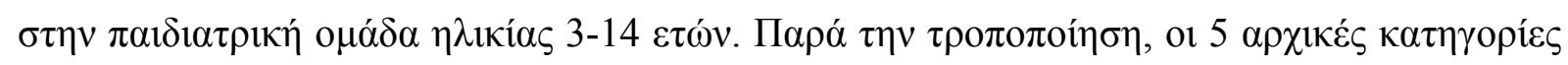




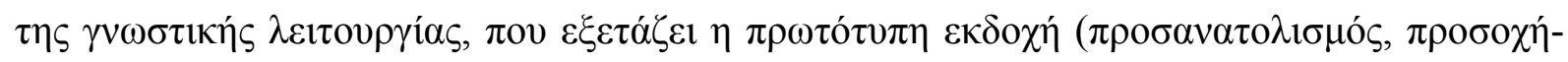

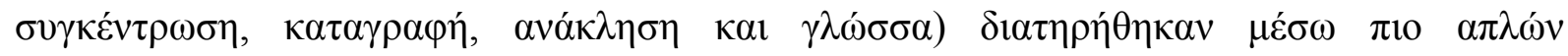

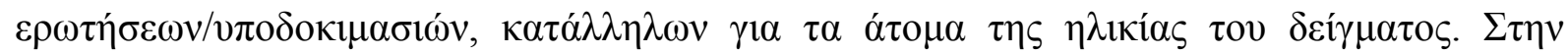

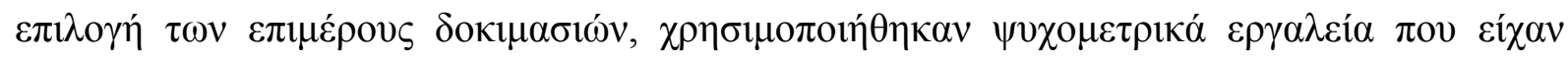

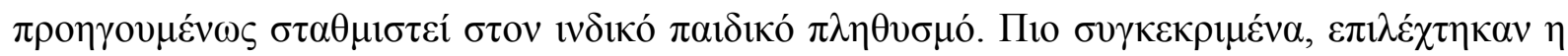
$\kappa \lambda i ́ \mu \alpha \kappa \alpha$ Behavioral Assessment Scale for Indian Children with Mental Retardation (BASIC-

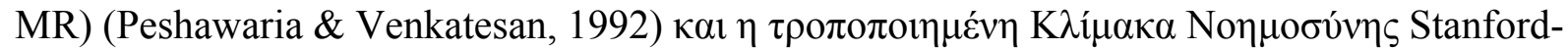

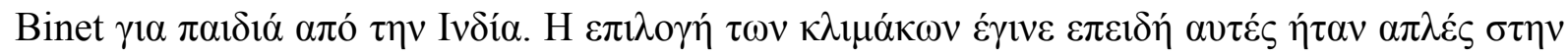

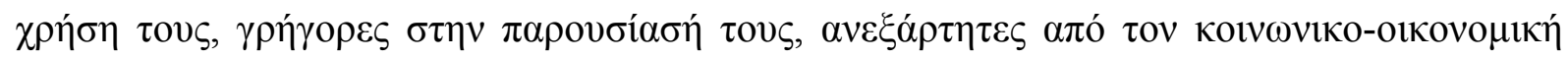

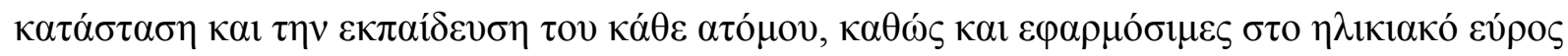

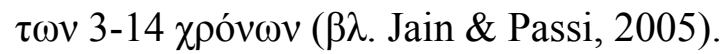

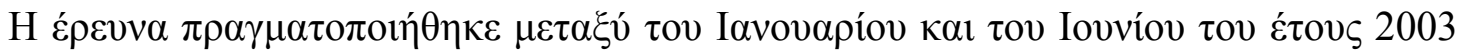

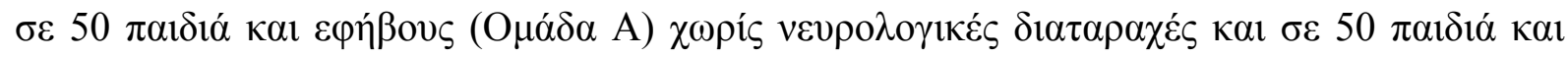

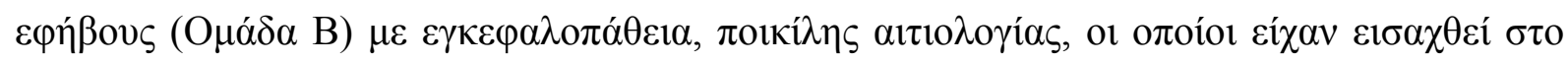

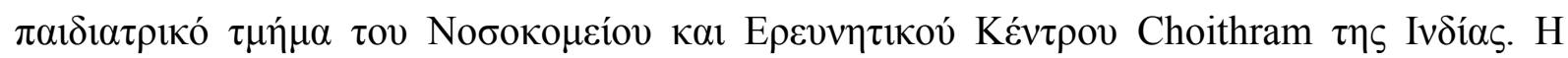

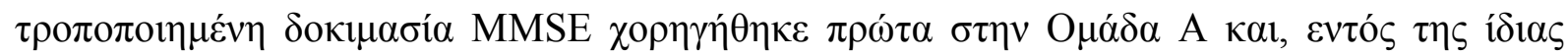

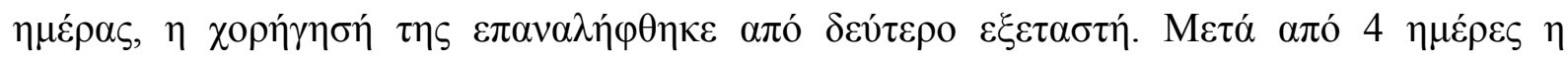

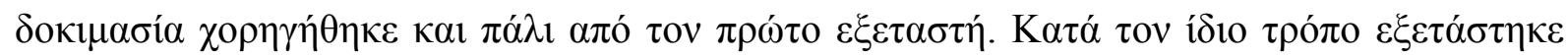

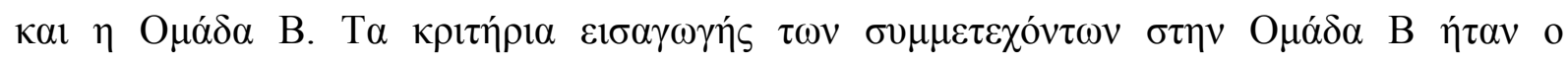

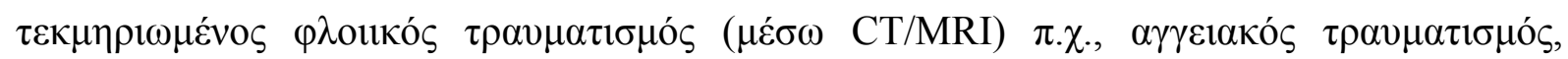

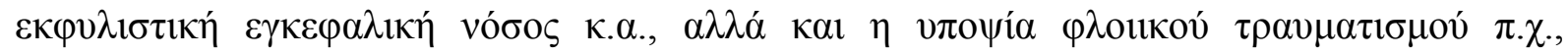

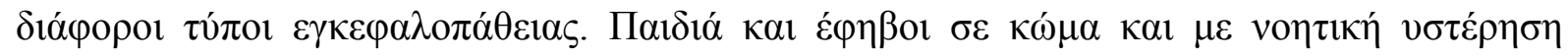
$\alpha \pi о \kappa \lambda \varepsilon i ́ \sigma \tau \eta \kappa \alpha \nu$.

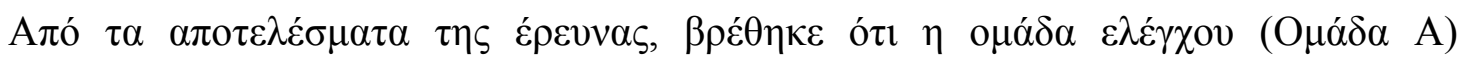

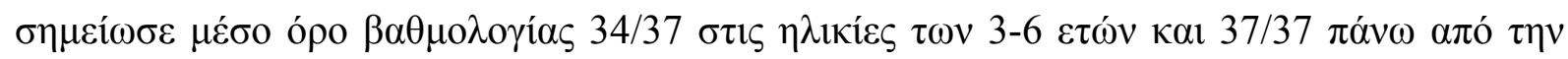




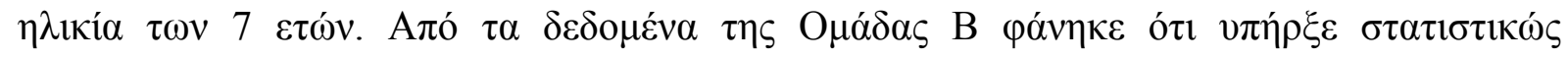

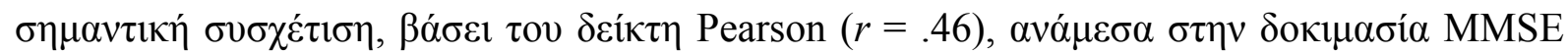

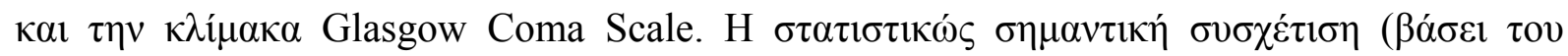

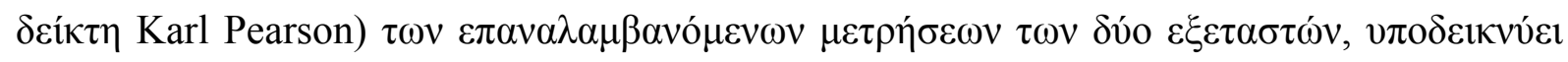

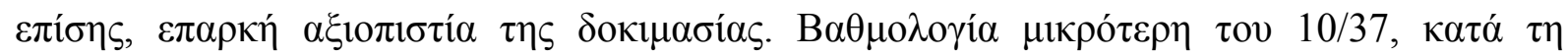

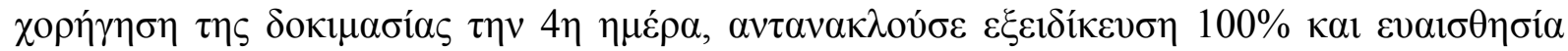

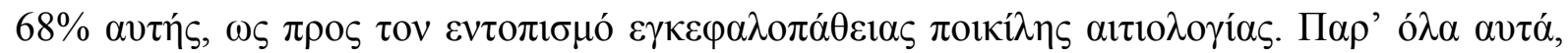

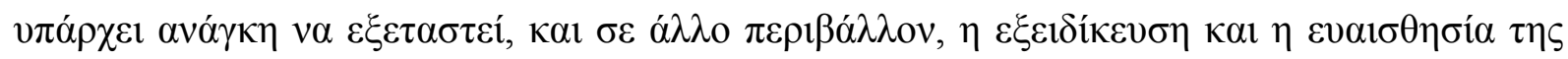

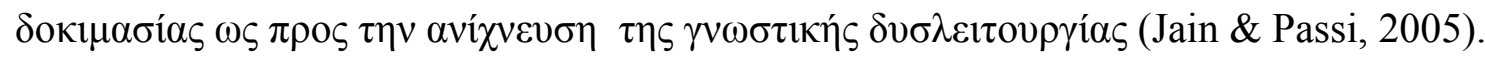

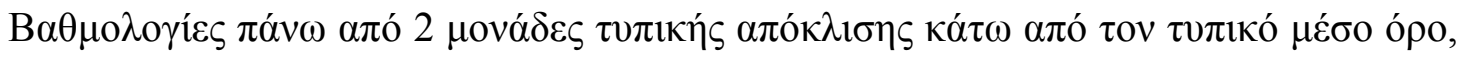

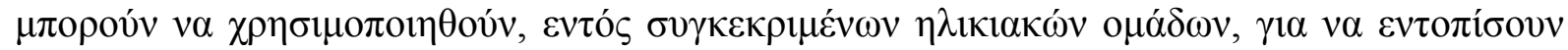

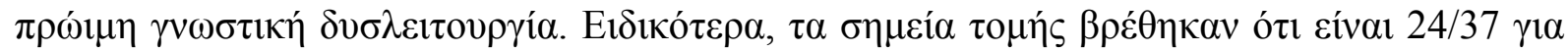

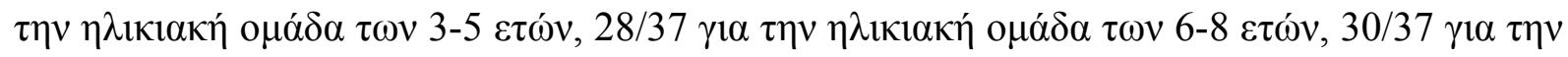

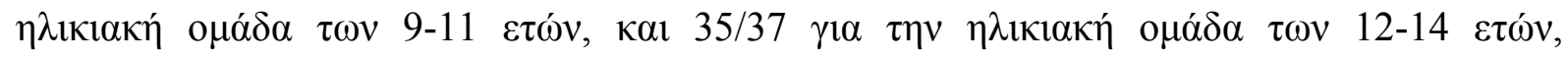

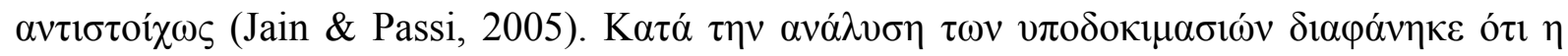

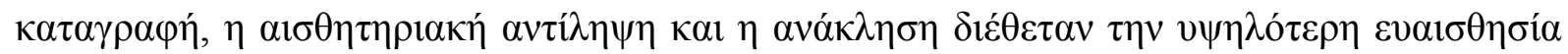

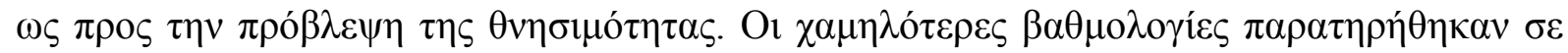

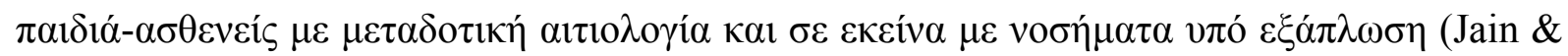
Passi, 2005).

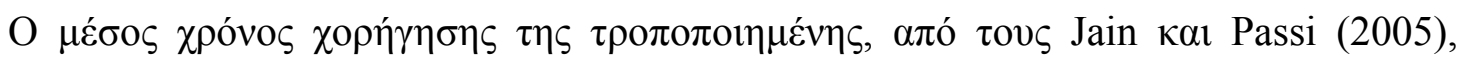

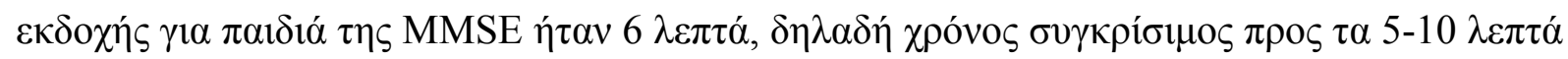

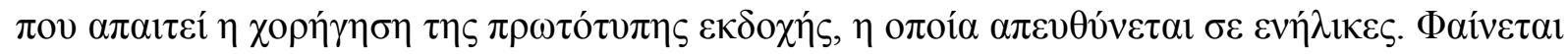

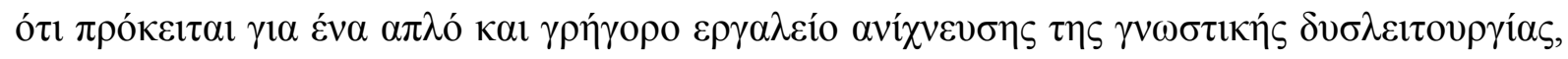

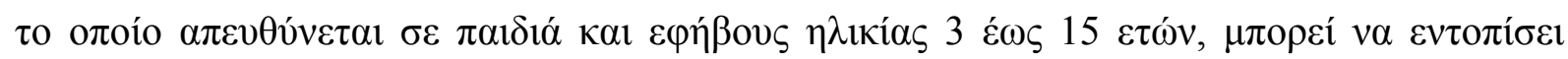

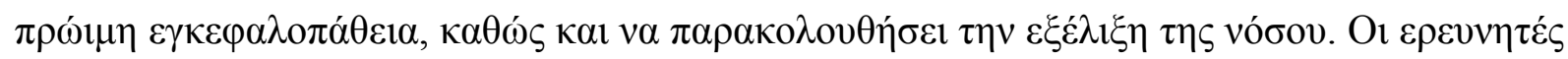




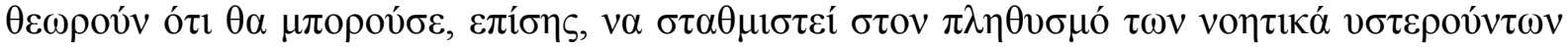
$\pi \alpha \imath \delta \iota \omega ́ v$ (Jain \& Passi, 2005). 


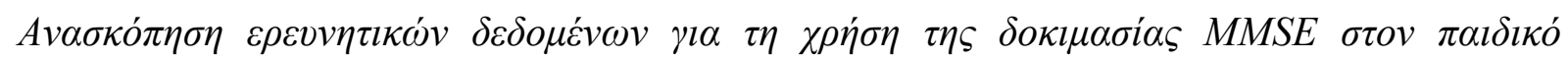
$\pi \lambda \eta \theta v \sigma \mu o ́ ~ \tau \eta \varsigma ~ I \sigma \pi \alpha v i ́ \alpha \varsigma$

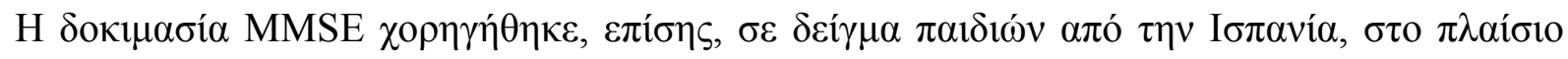

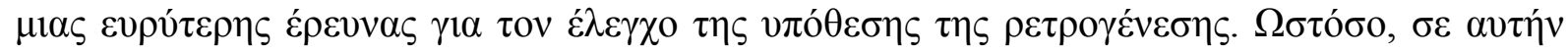

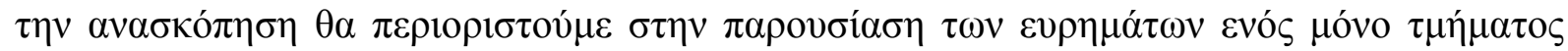

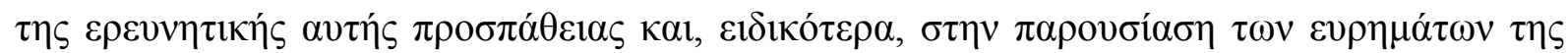

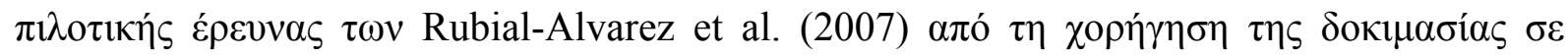

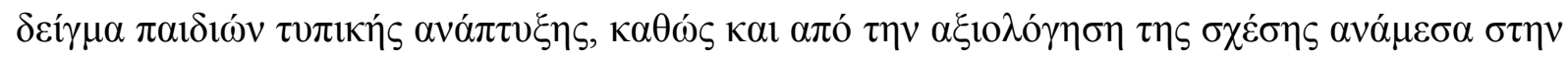

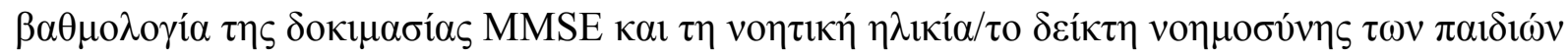

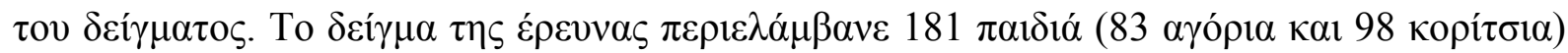

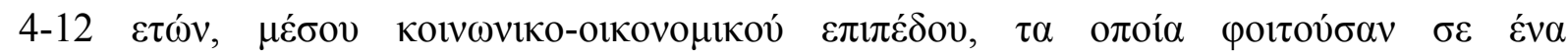

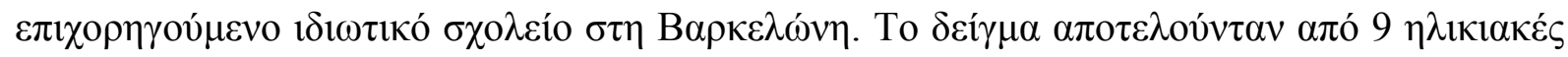

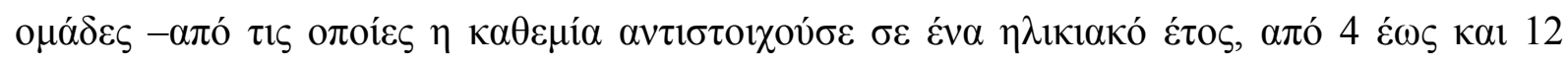

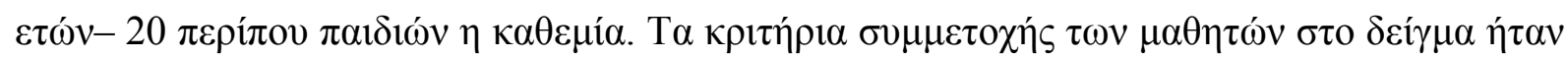

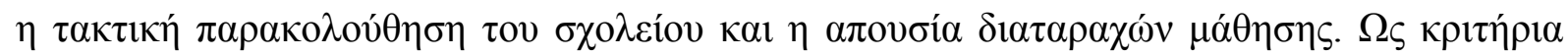

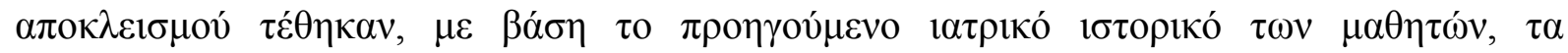

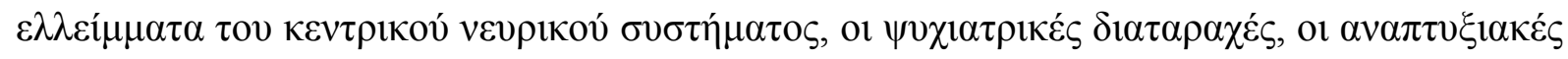

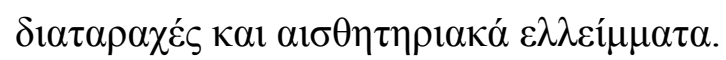

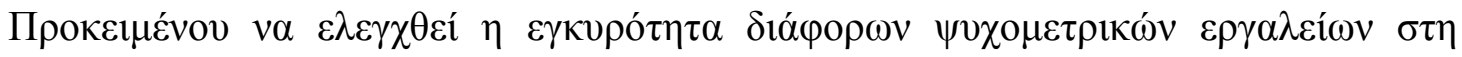

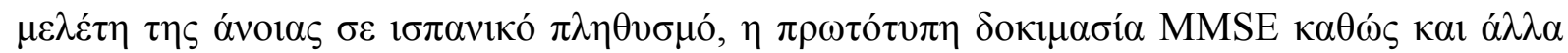

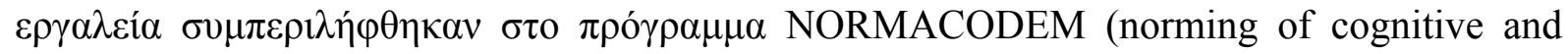

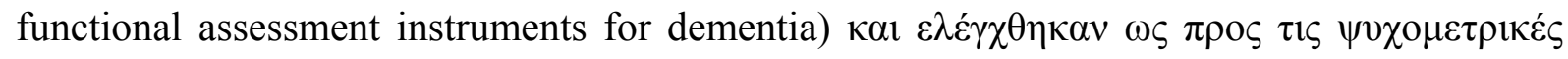

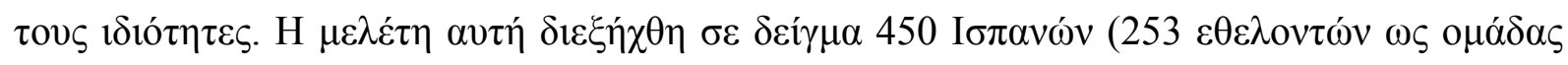

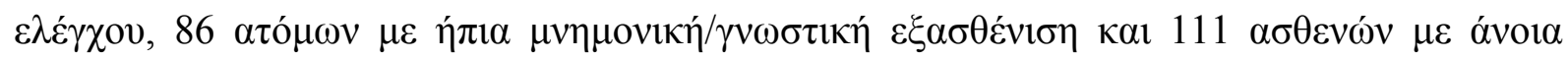




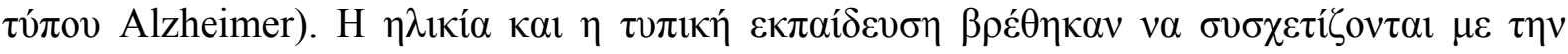

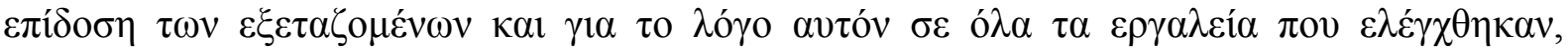

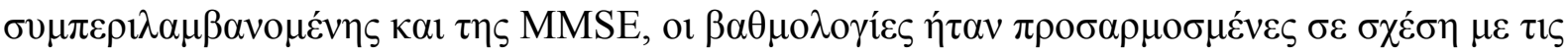

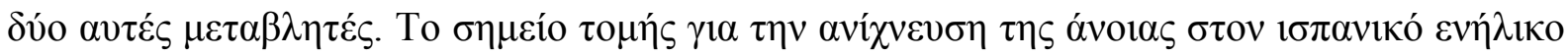

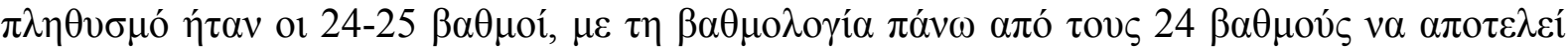

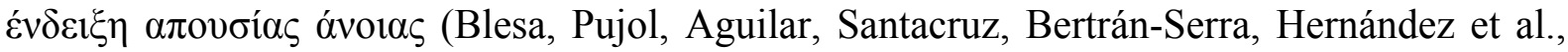
2001).

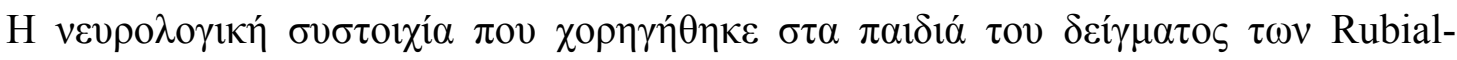
Alvarez et al. (2007) a

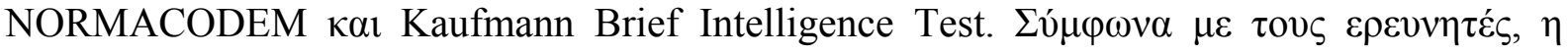

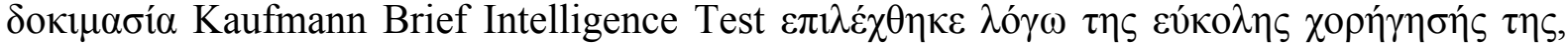

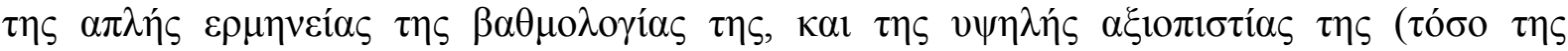

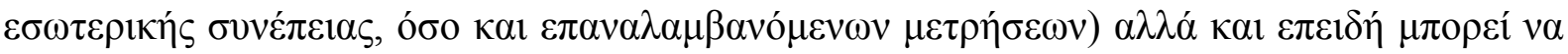

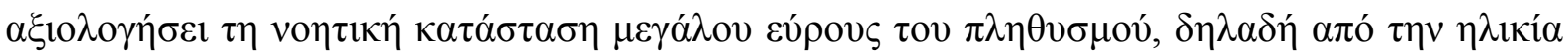
$\tau \omega \nu 4 \varepsilon \dot{\varepsilon} \omega \varsigma \kappa \alpha \iota \tau \omega \nu 90 \varepsilon \tau \omega \dot{\nu}$.

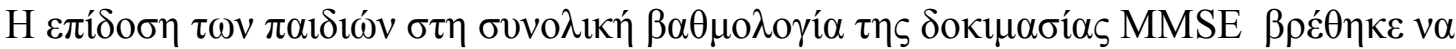

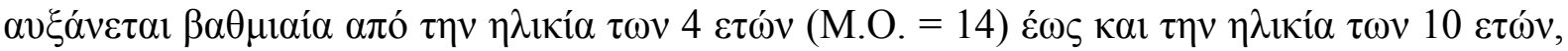

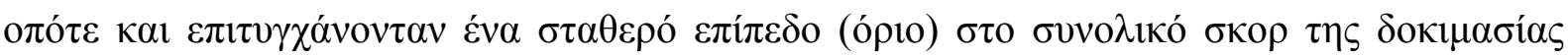

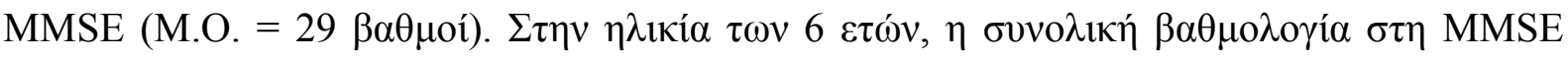

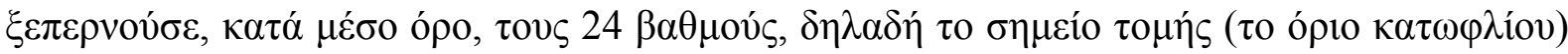

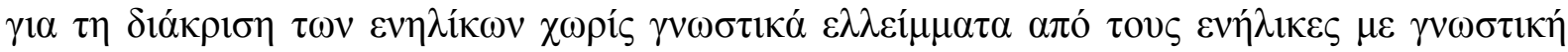
$\varepsilon ́ \kappa \pi \tau \omega \sigma \eta$ (Rubial-Alvarez et al., 2007).

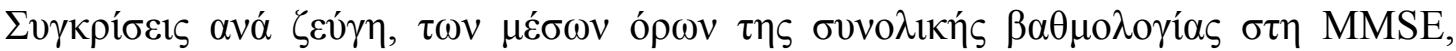

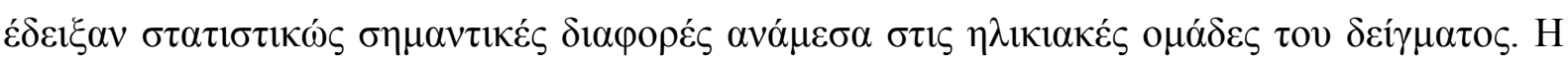

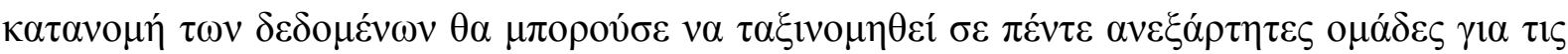




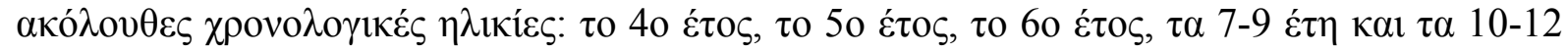

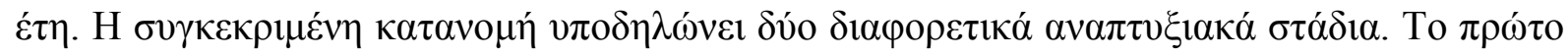

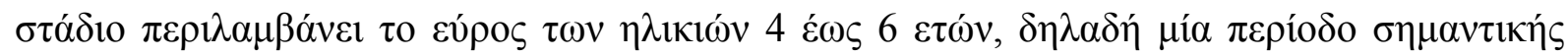

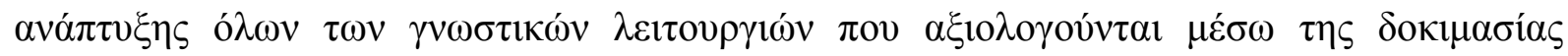

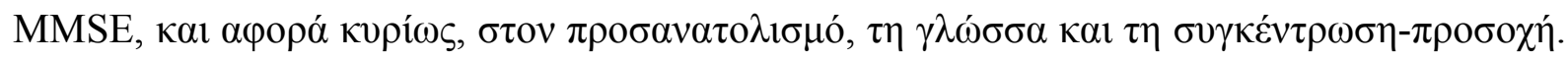

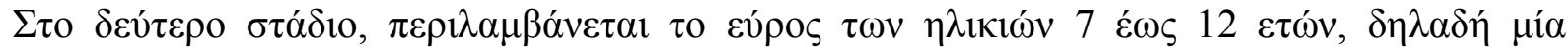

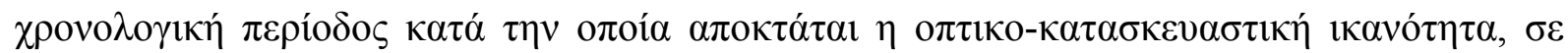

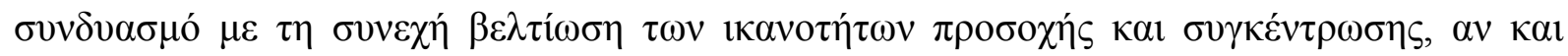

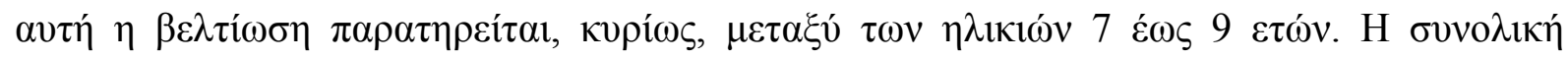

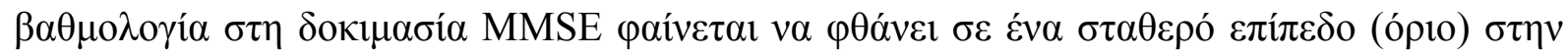

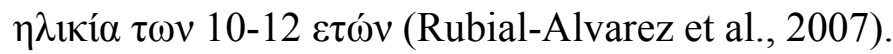

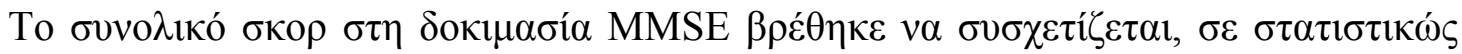

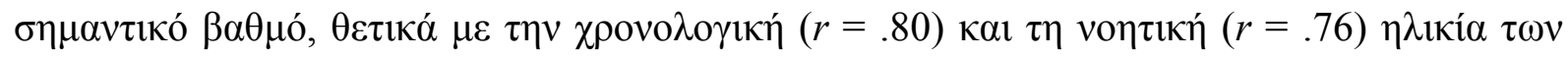

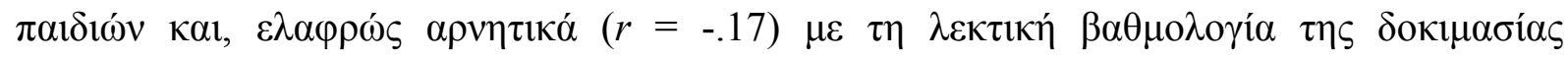

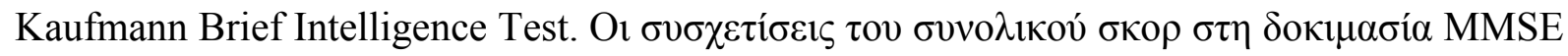

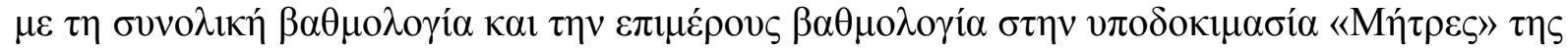

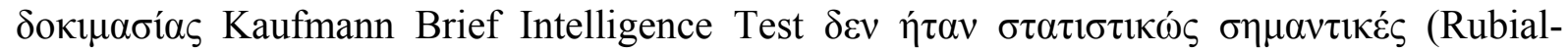
Alvarez et al., 2007).

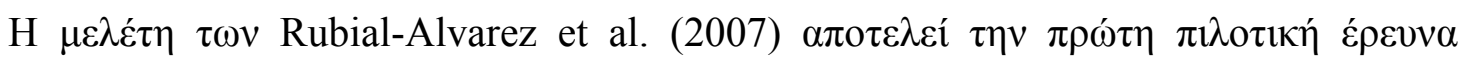

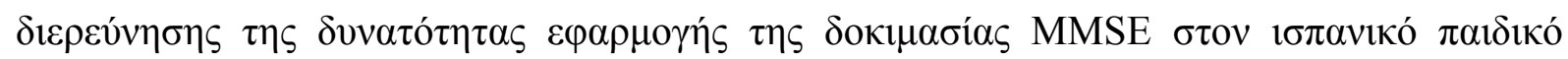

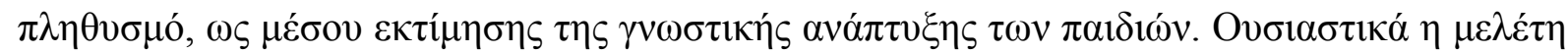

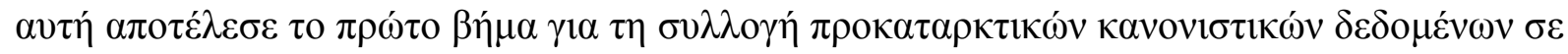

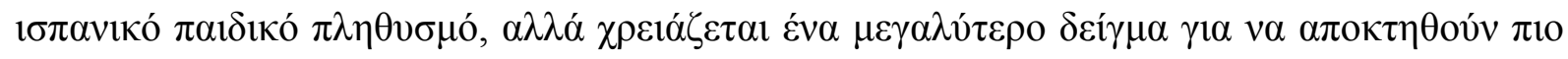

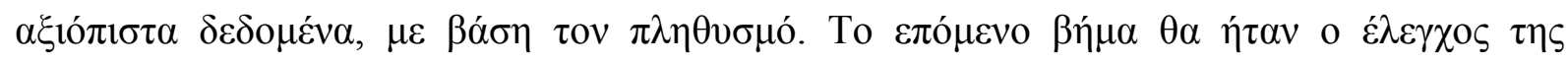




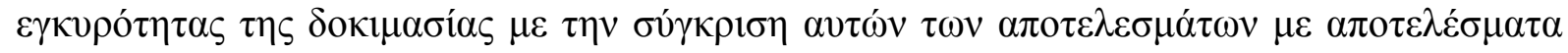

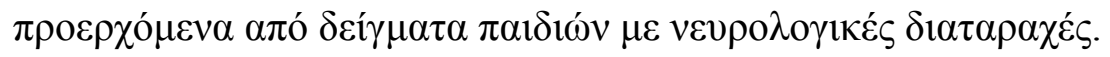

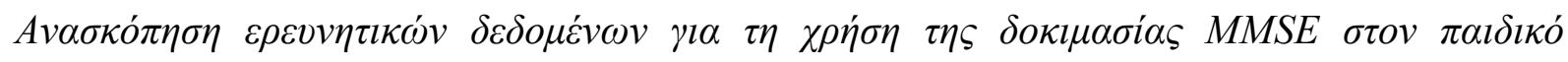

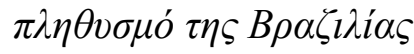

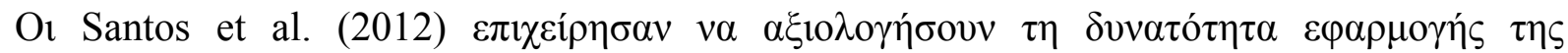

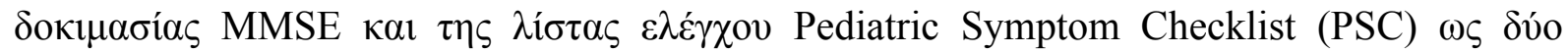

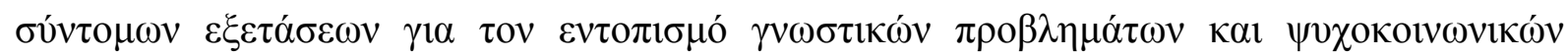

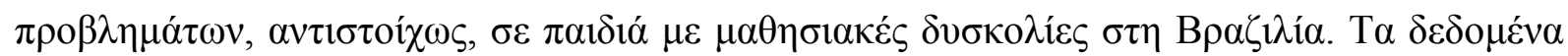

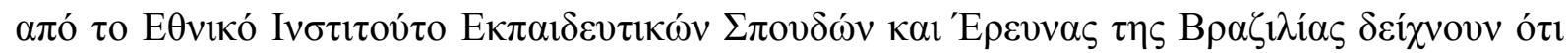

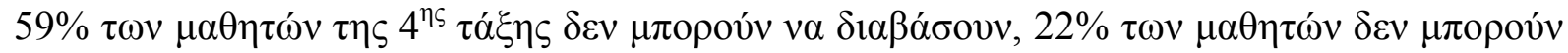

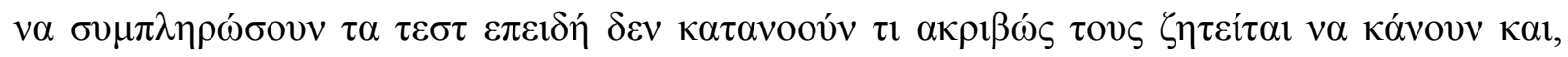

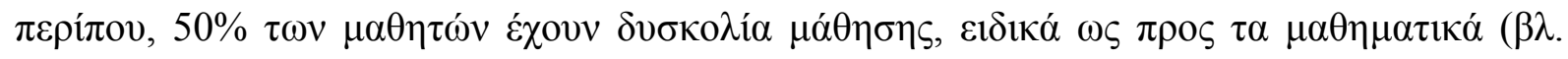

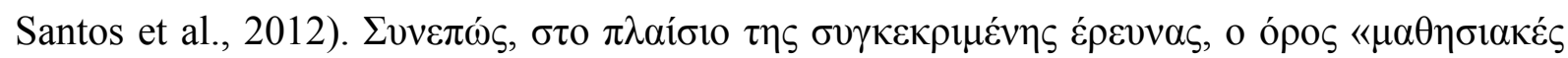

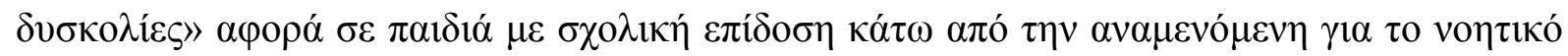

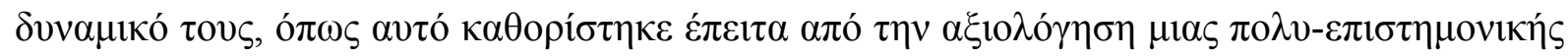

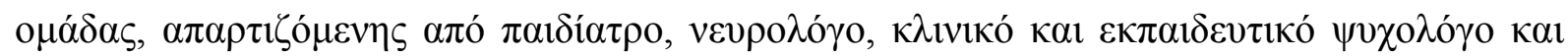

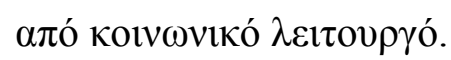

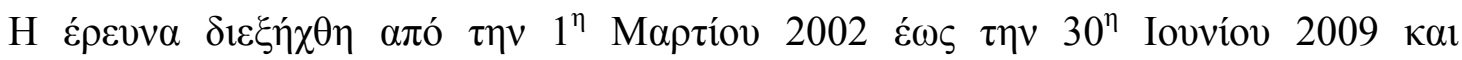

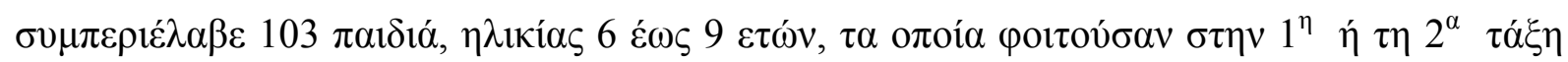

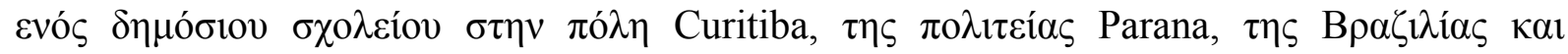

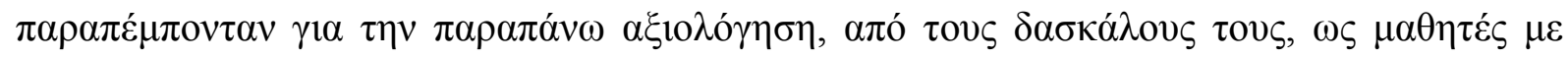

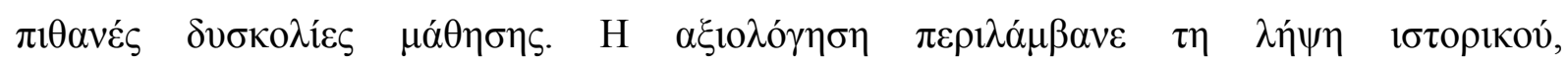

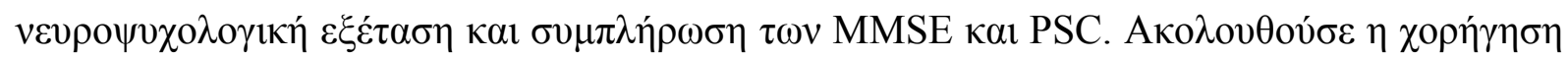




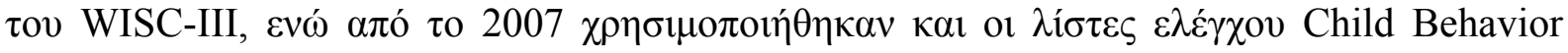

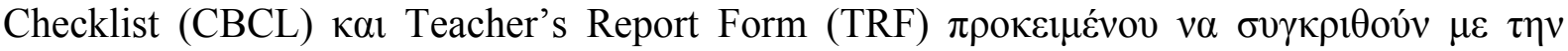
PSC.

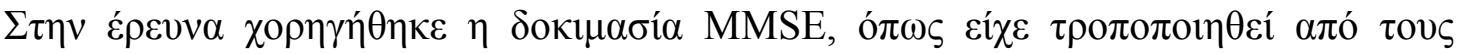

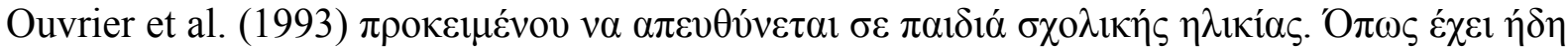

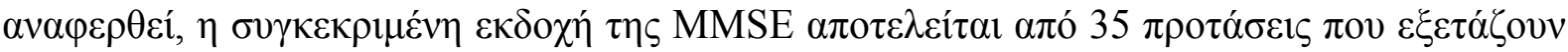

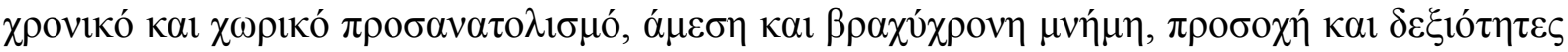

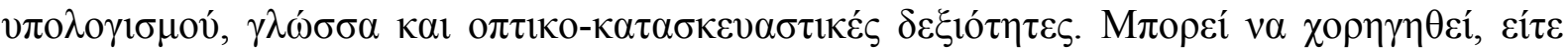

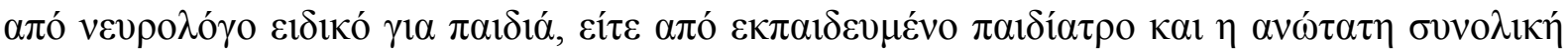

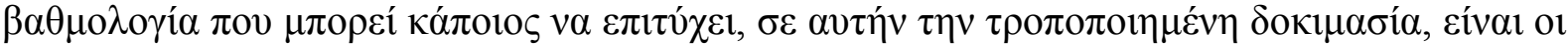

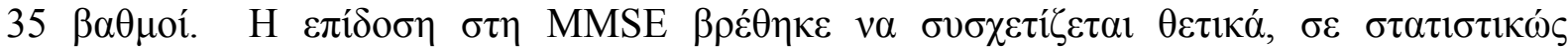

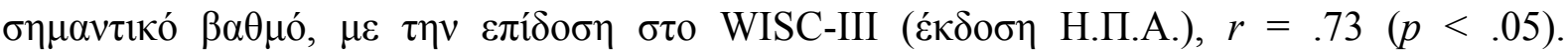

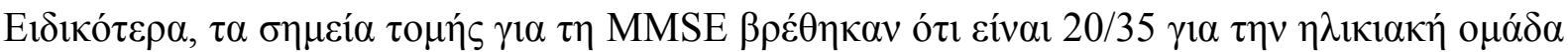

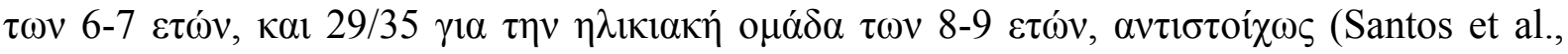
2012).

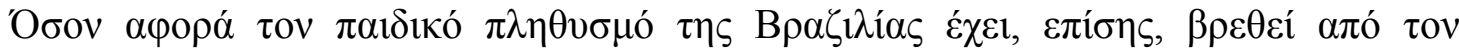

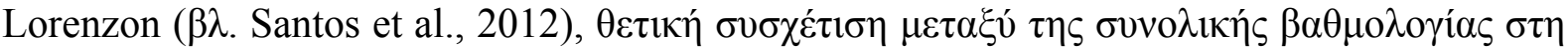

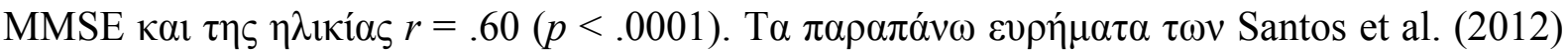

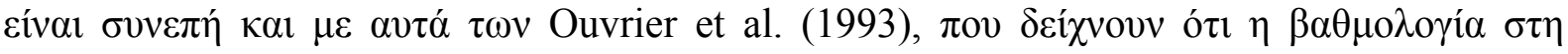

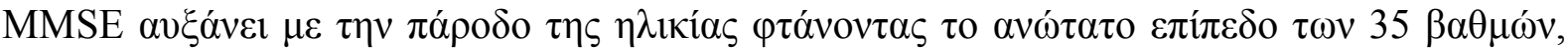

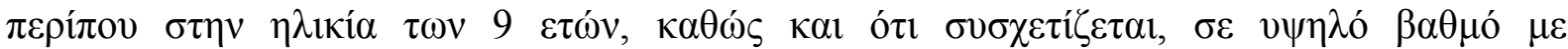

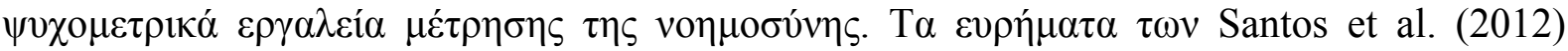

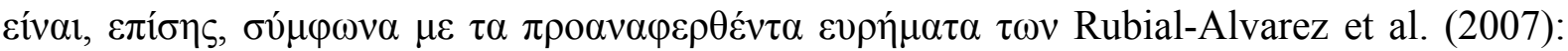

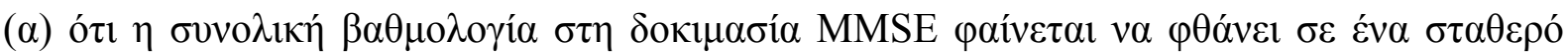

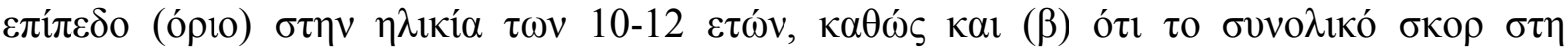




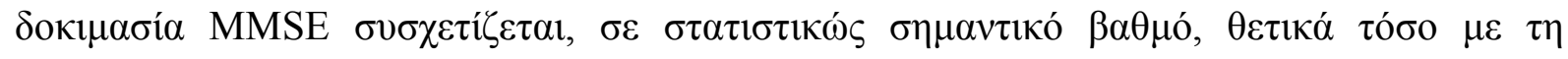

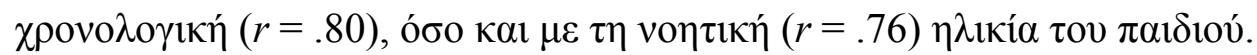

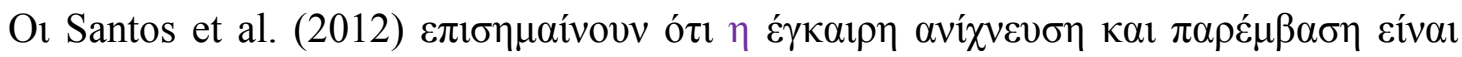

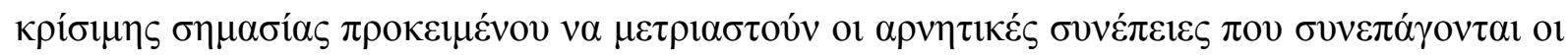

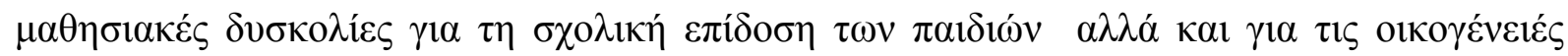

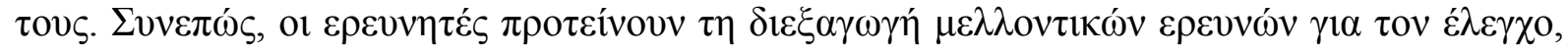

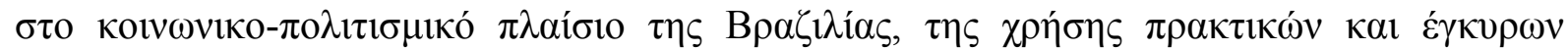

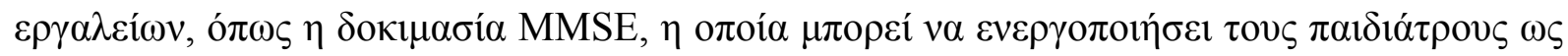

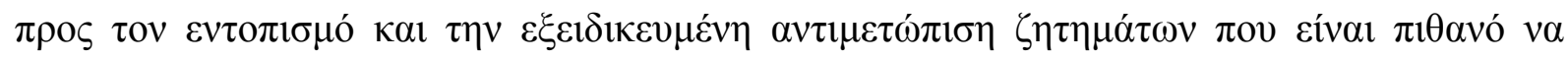

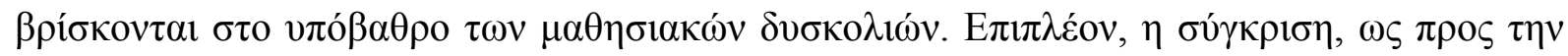
$\varepsilon \pi i ́ \delta o \sigma \eta \sigma \tau$ MMSE, $\mu \varepsilon \tau \alpha \xi \dot{v} \pi \alpha 1 \delta เ \omega ́ v \mu \varepsilon \mu \alpha \theta \eta \sigma 1 \alpha \kappa \varepsilon \dot{\zeta} \delta v \sigma \kappa o \lambda i ́ \varepsilon \varsigma \kappa \alpha \imath \alpha \sigma \theta \varepsilon v \omega ́ v \mu \varepsilon$ ATA, $\theta \alpha$ $\mu \pi$

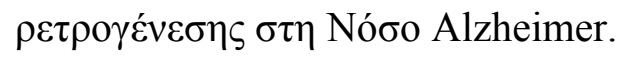

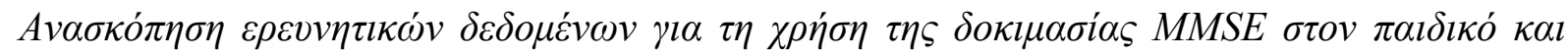
$\varepsilon \varphi \eta \beta \imath \kappa o ́ ~ \pi \lambda \eta \theta v \sigma \mu o ́ ~ \tau \eta \varsigma ~ E \lambda \lambda \alpha ́ \delta \alpha \varsigma$

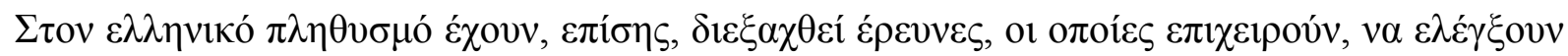

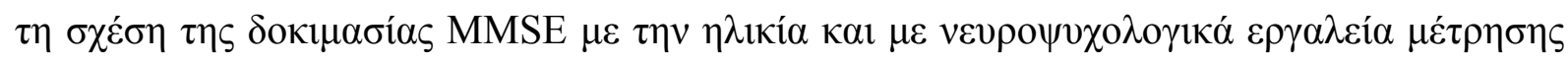

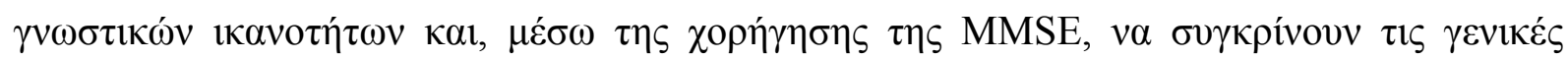

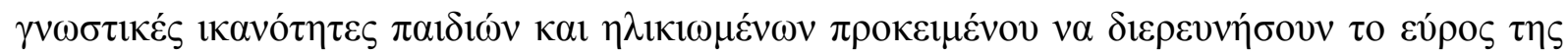

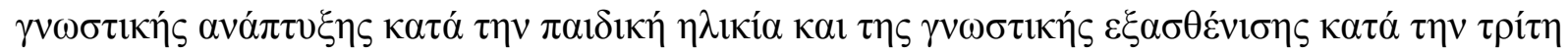

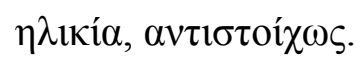

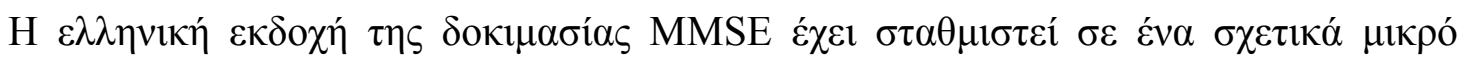

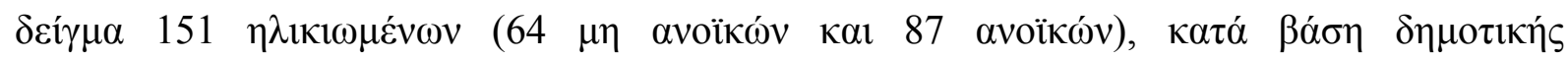




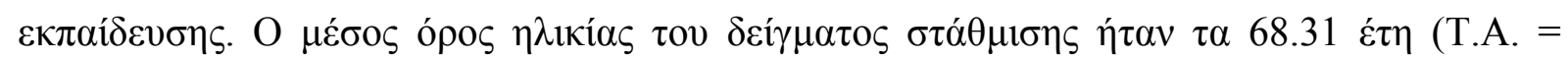

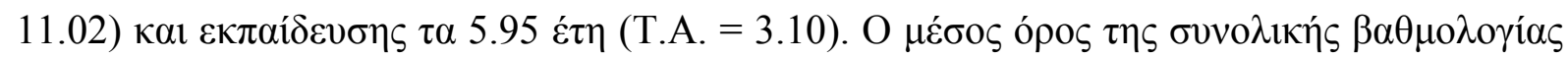

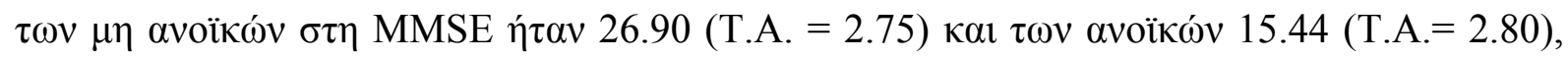

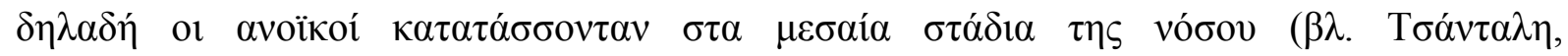

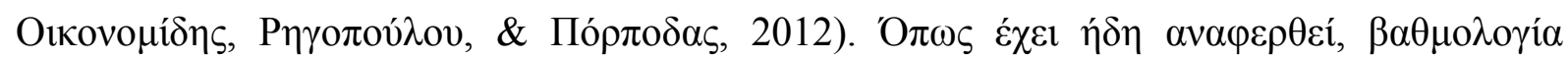

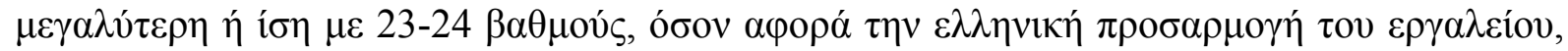

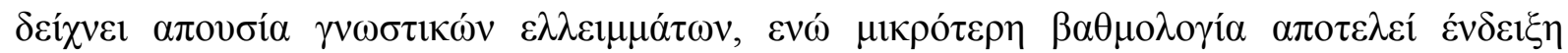

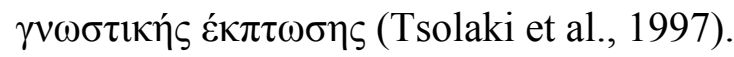

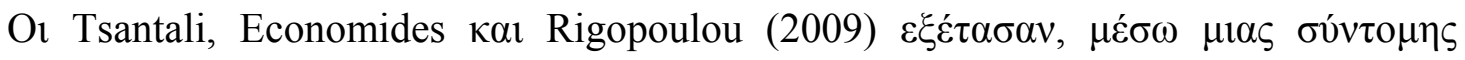

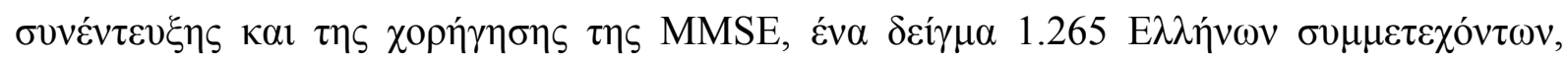

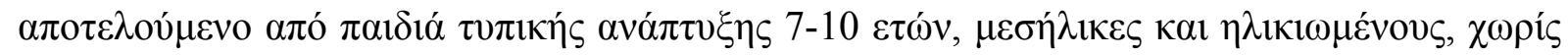

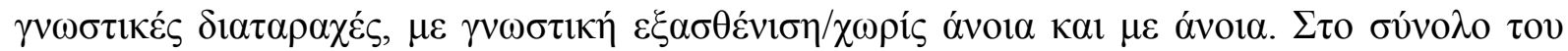

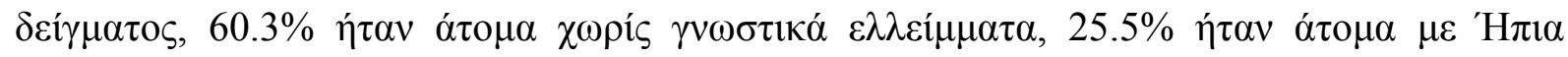

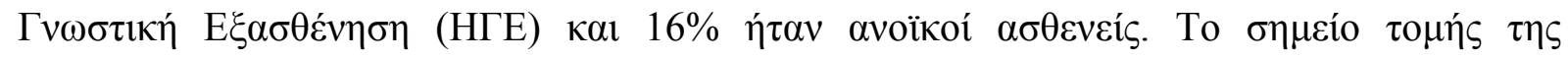

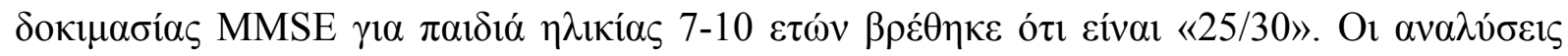

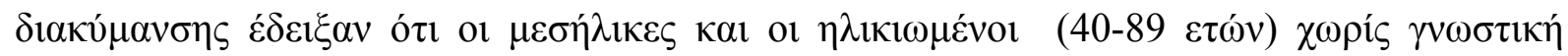

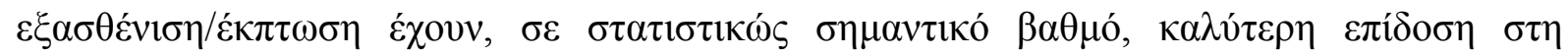

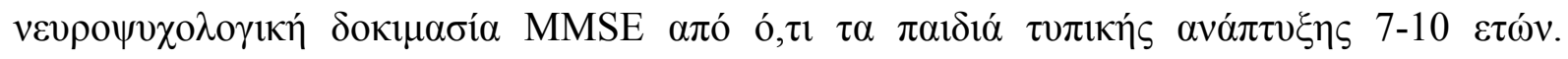

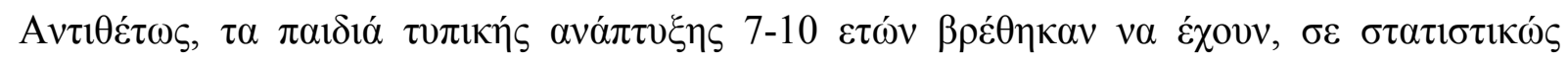

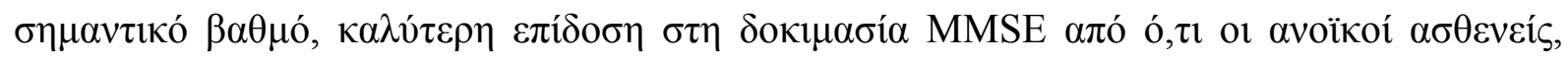

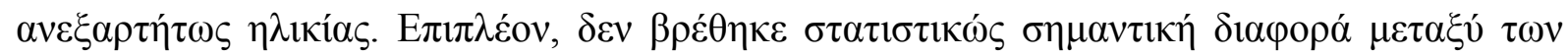

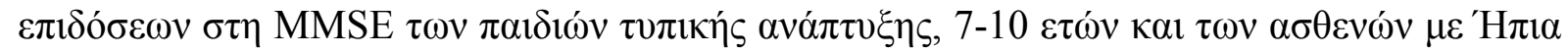

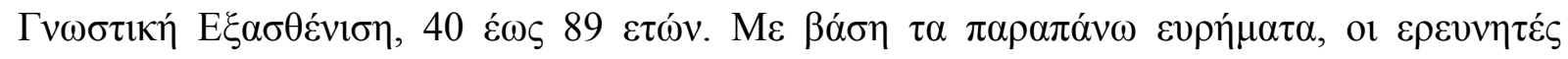

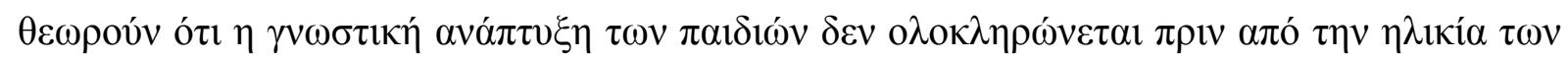

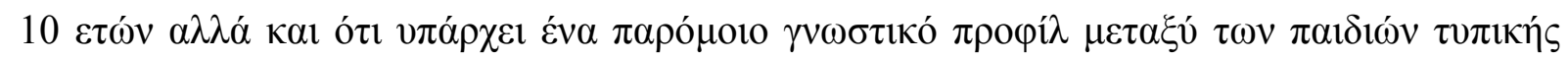




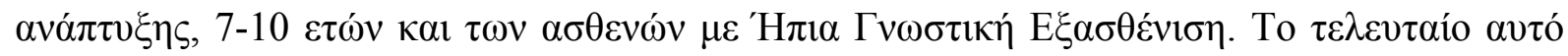

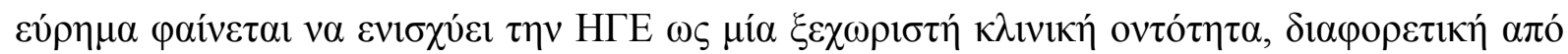

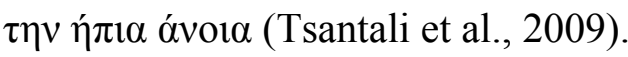

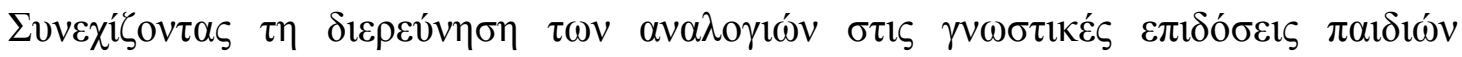

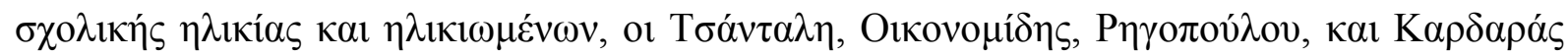

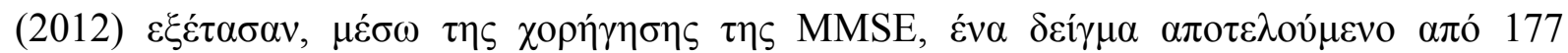

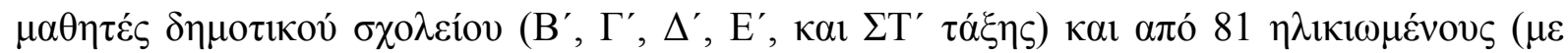

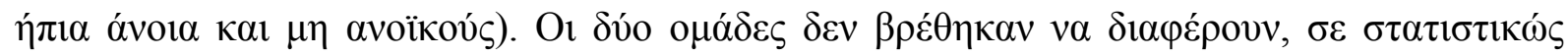

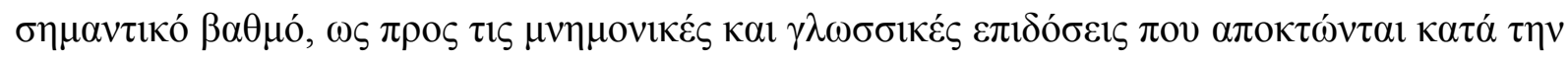

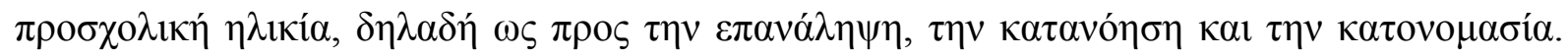

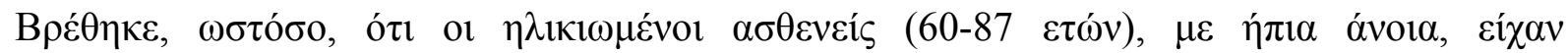

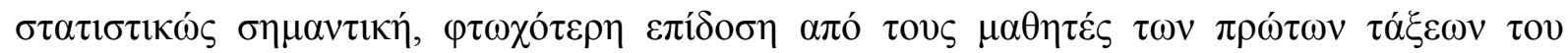

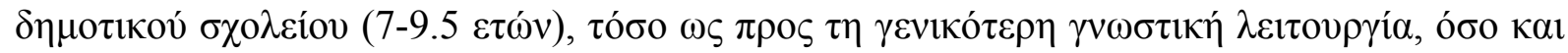

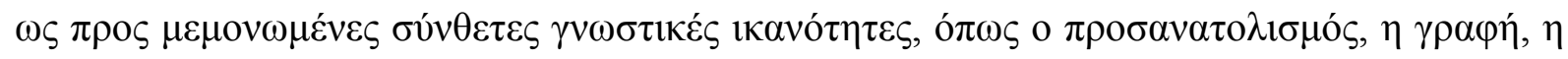

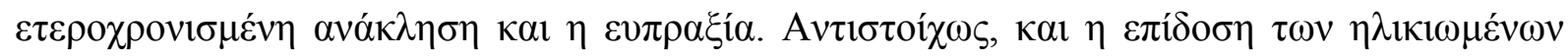

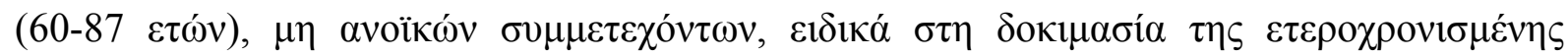

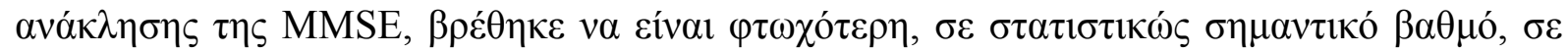

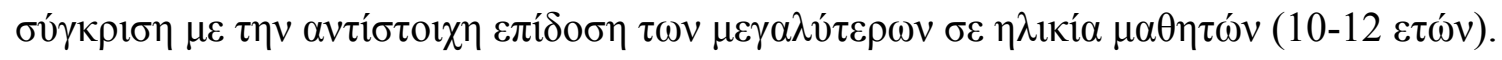

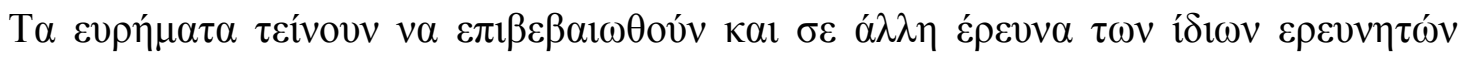

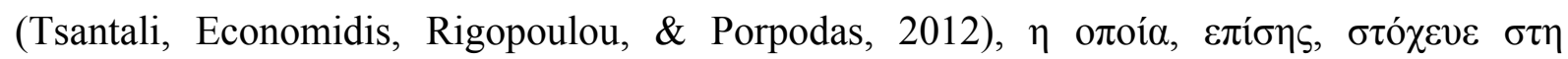

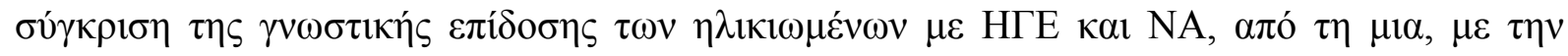

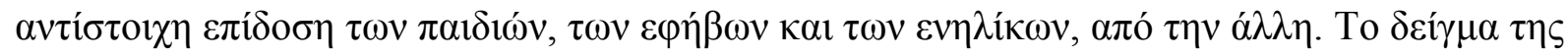

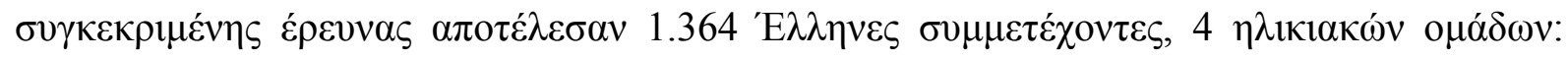

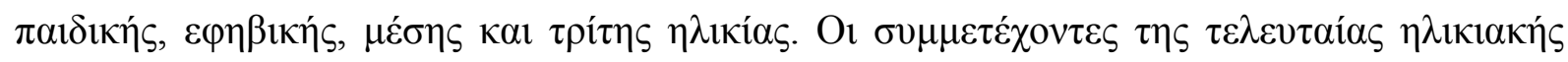

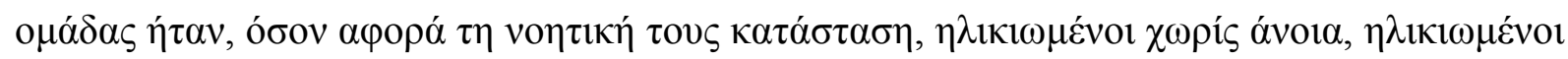




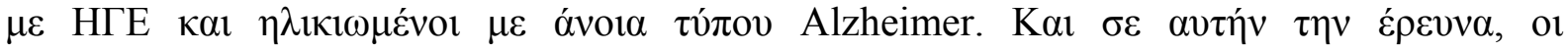

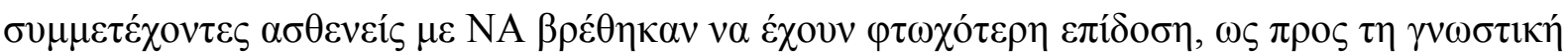

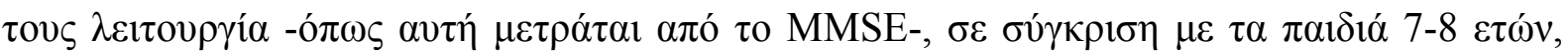

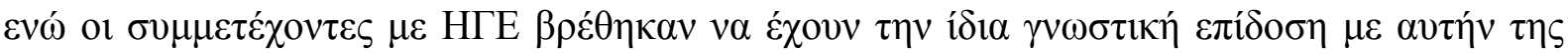

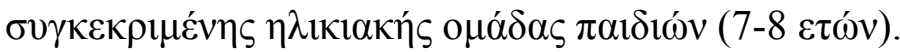

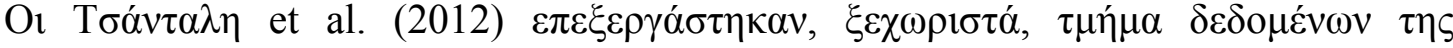

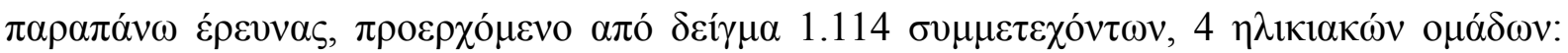

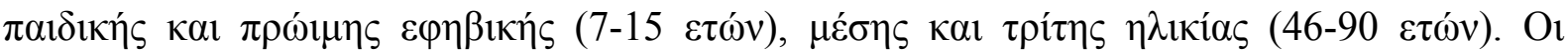

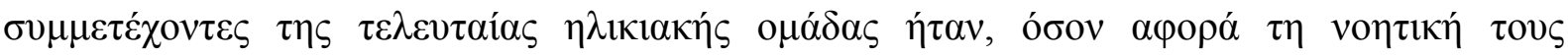

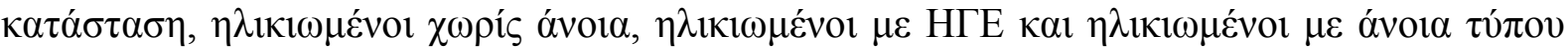

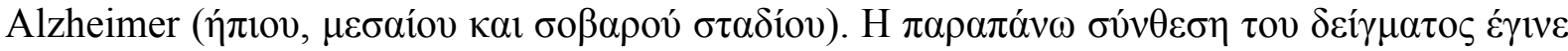

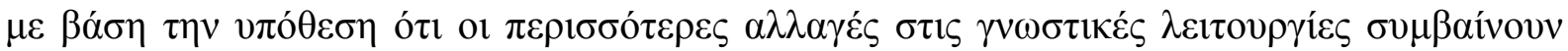

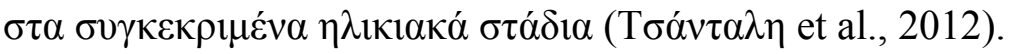

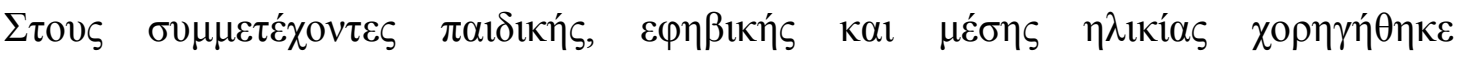

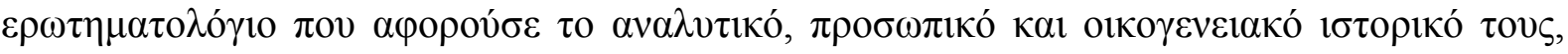

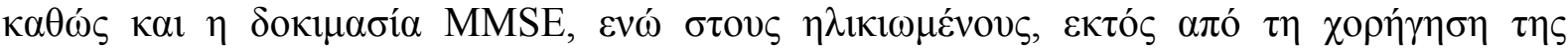

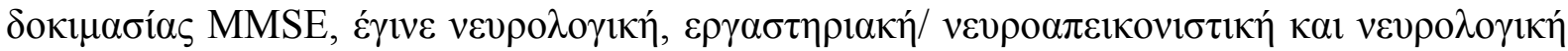

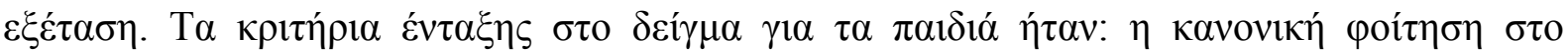

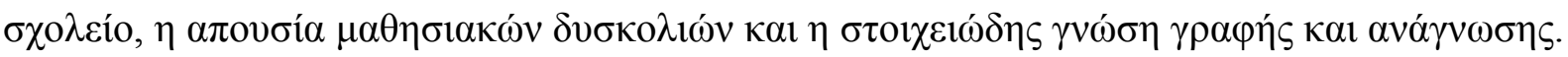

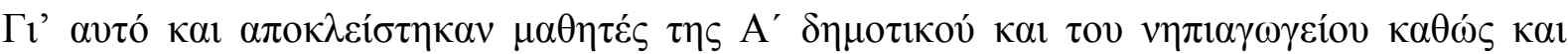

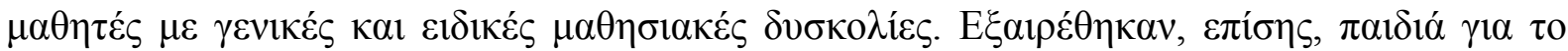

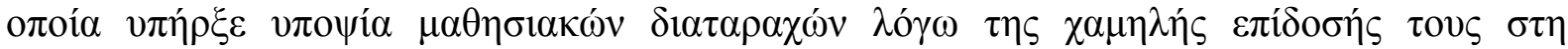

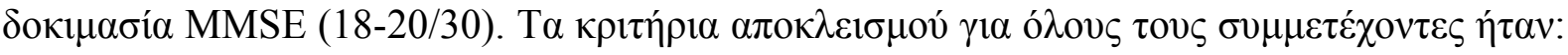

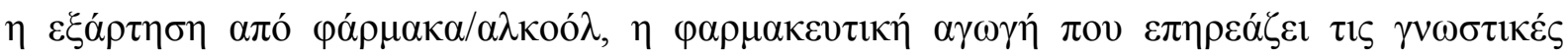

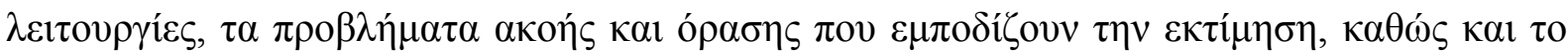




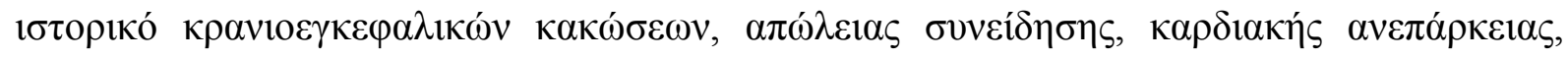

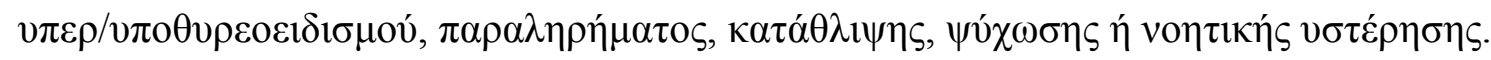

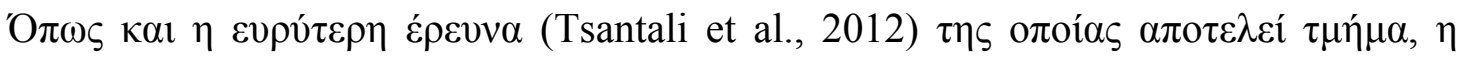

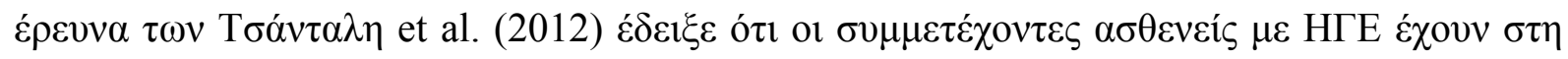

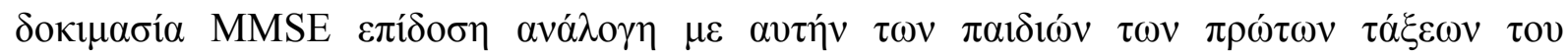

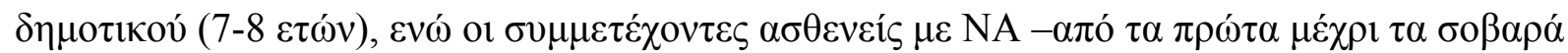

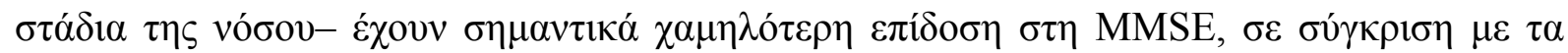

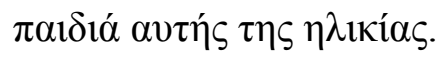

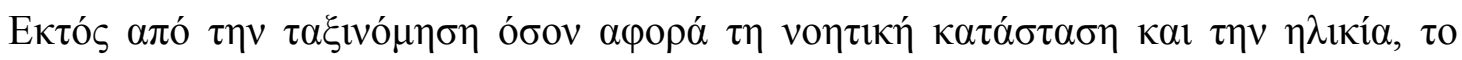

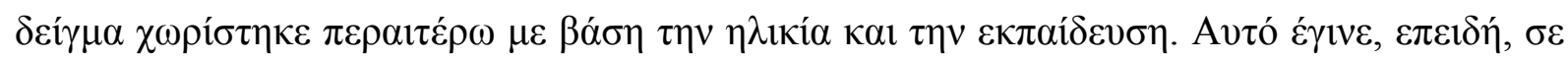

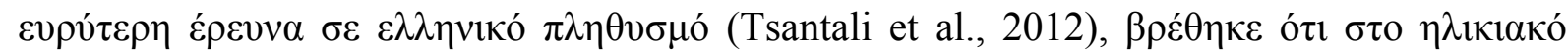

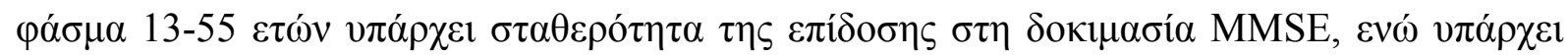

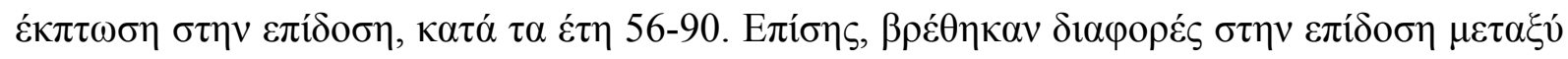

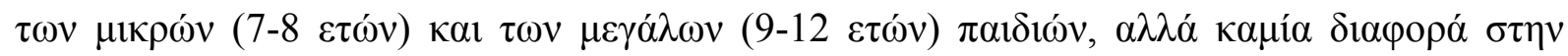

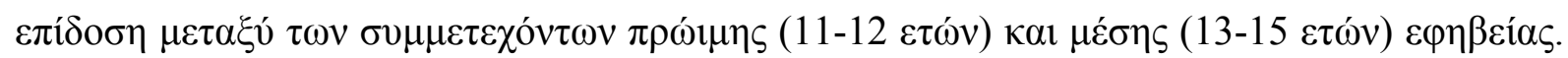

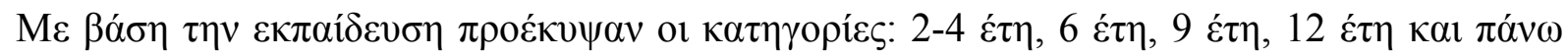

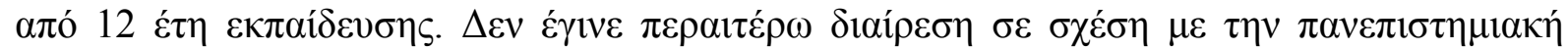

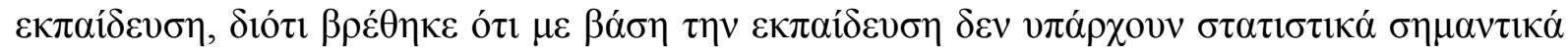

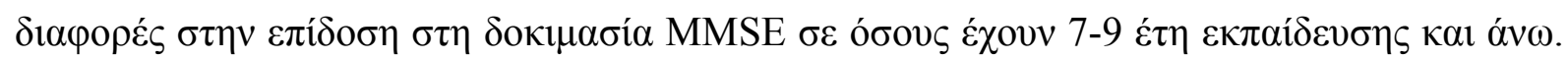

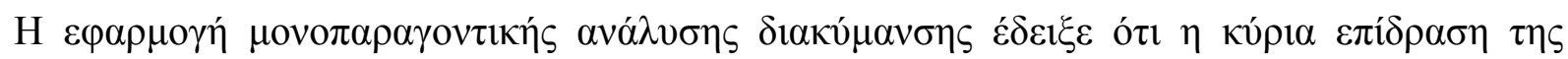

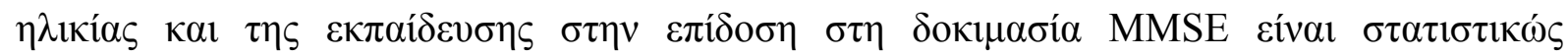

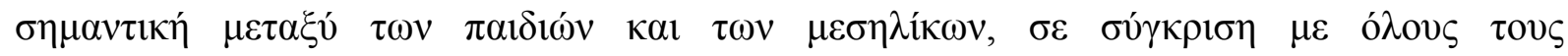

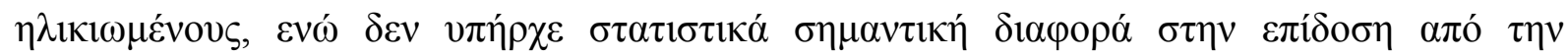

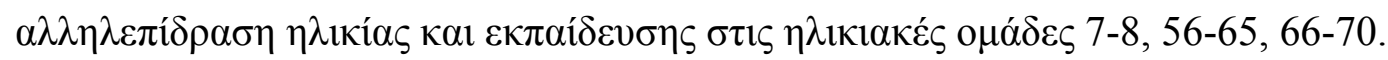




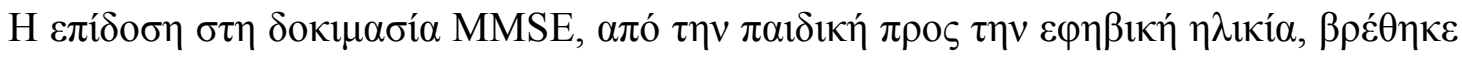

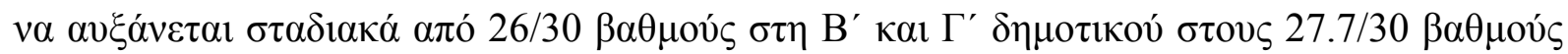

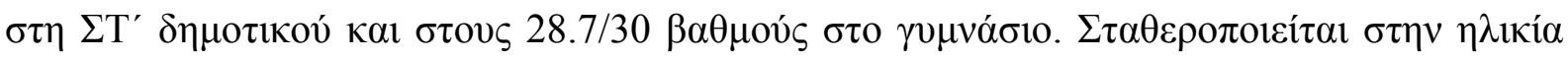

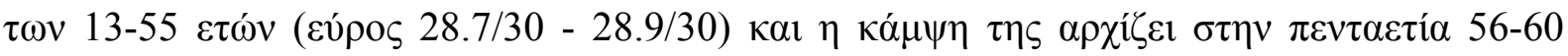

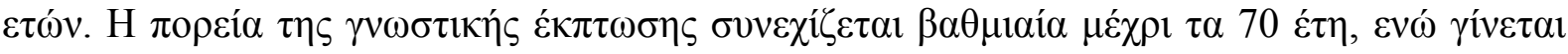

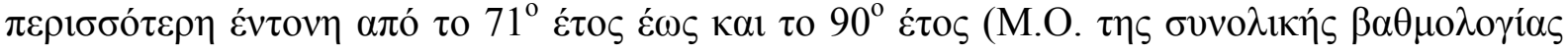

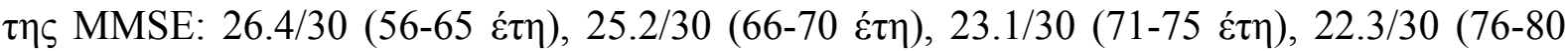

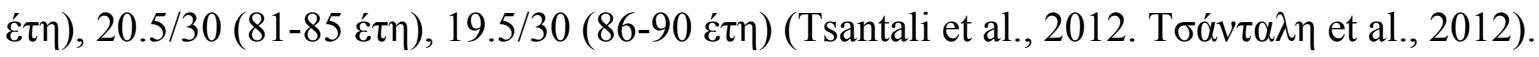

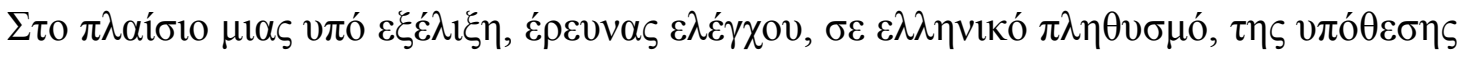

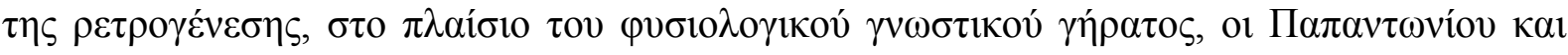
Mwpoï̌tov (2012. Dinou, Katsadima, Savvidou, Foutsitzi, Moraitou, \& Papantoniou,

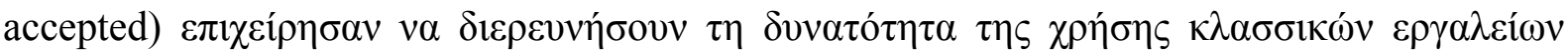

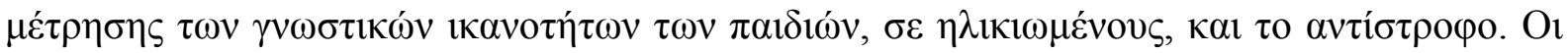

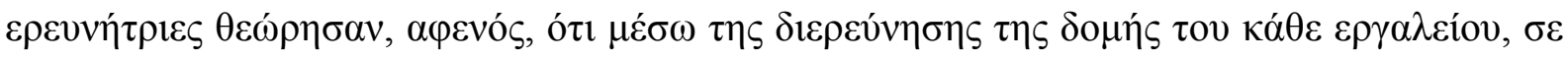

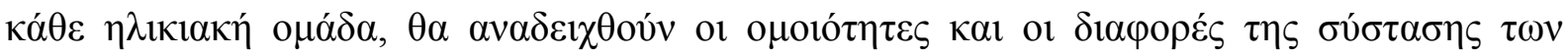

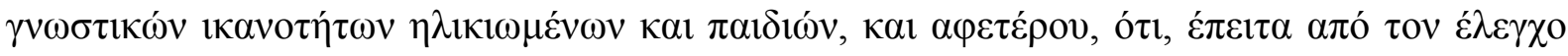

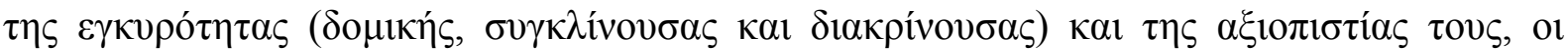

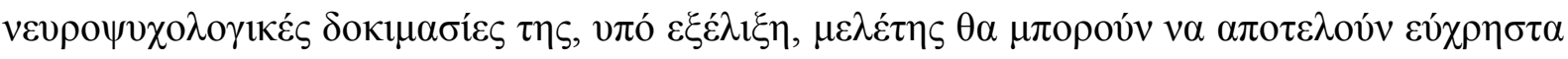

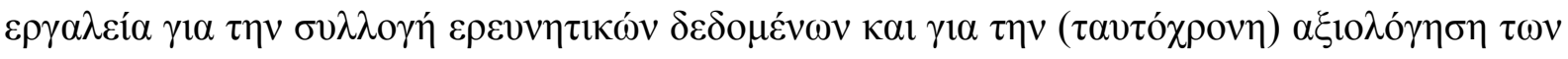

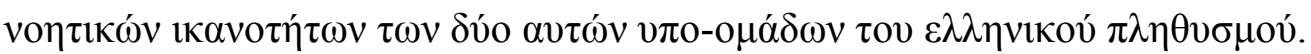

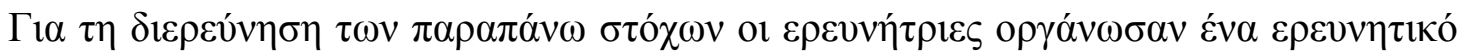

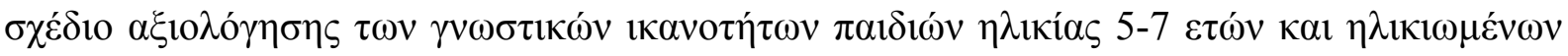

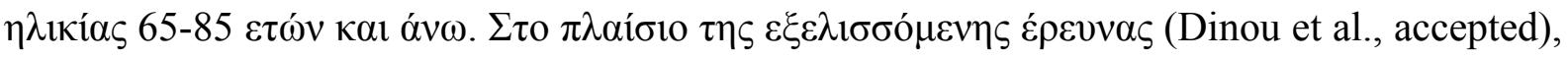

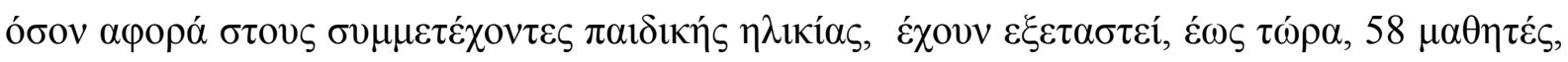

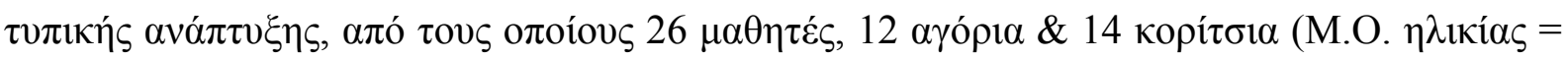




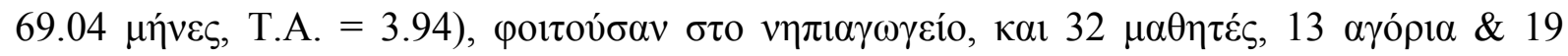

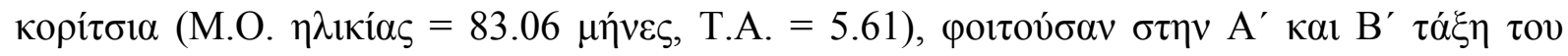

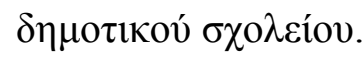

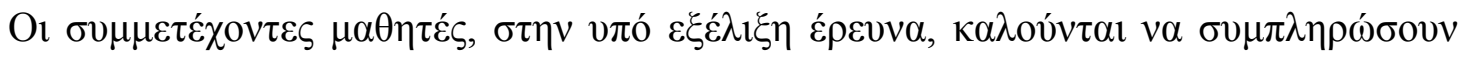

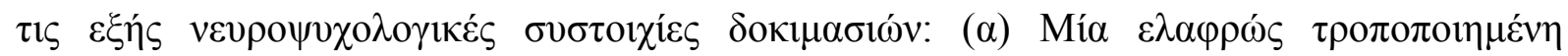

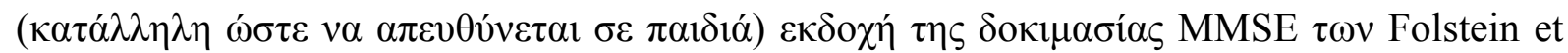

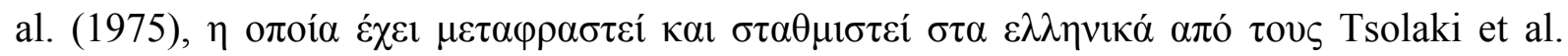

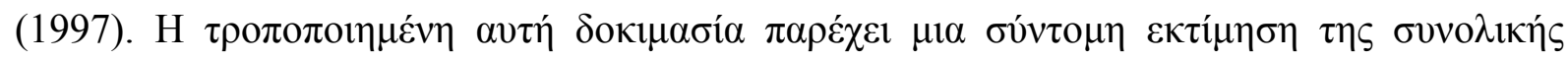

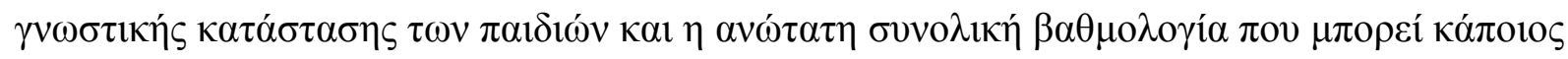

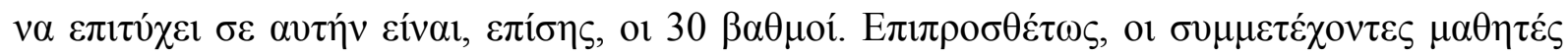

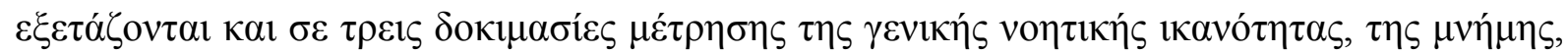

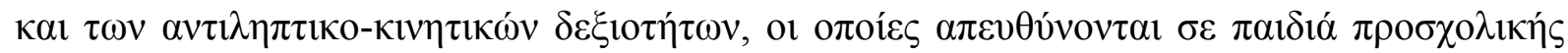

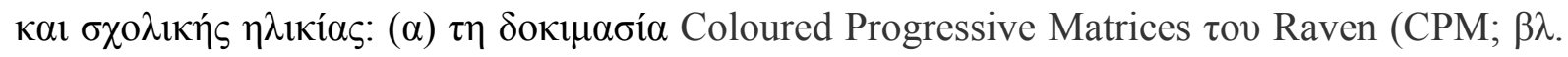

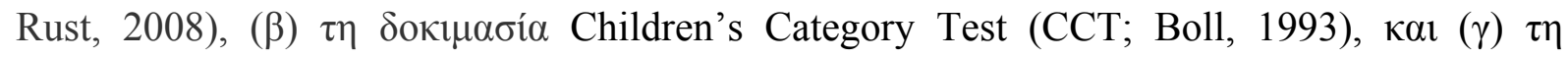

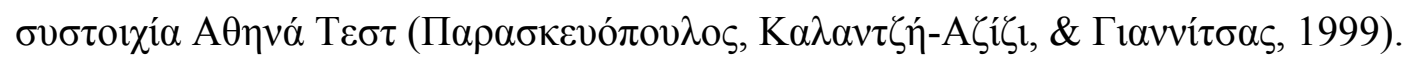

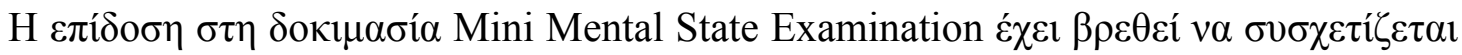

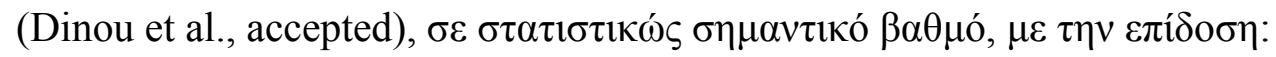

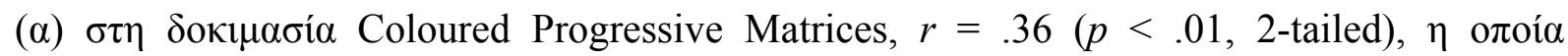

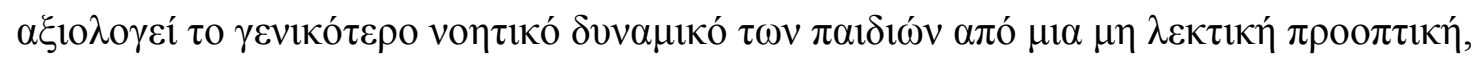

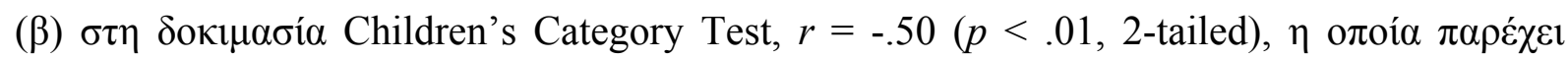

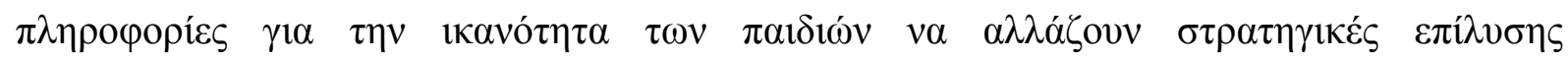

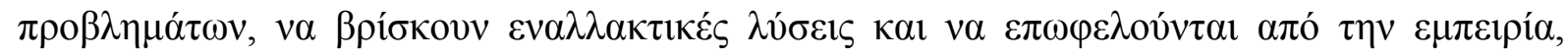

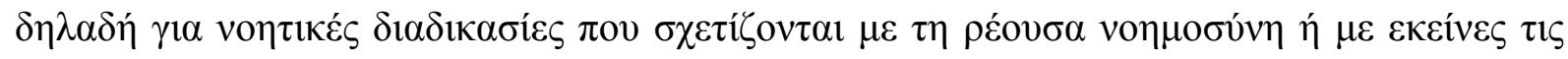

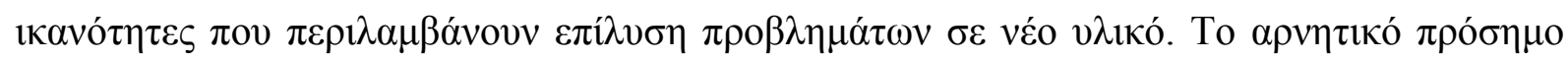




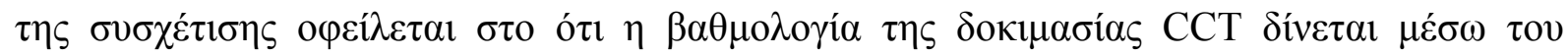

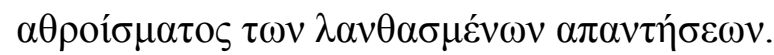

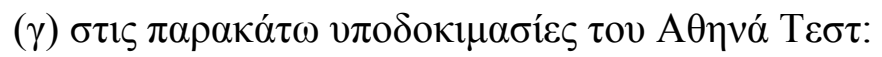

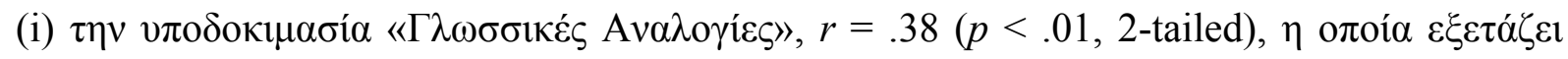

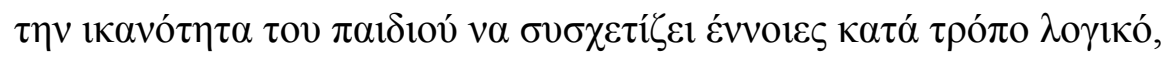

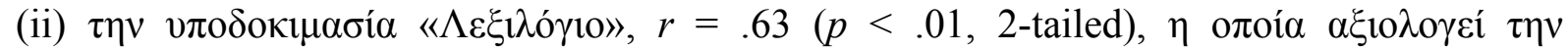

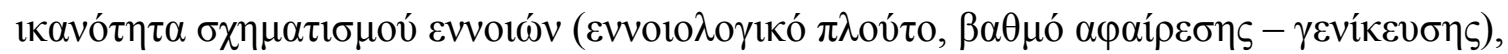

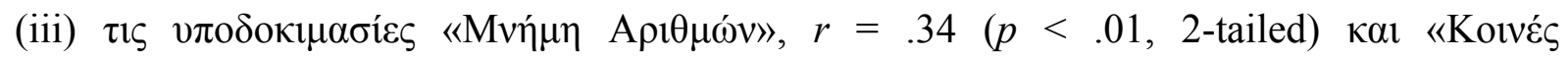

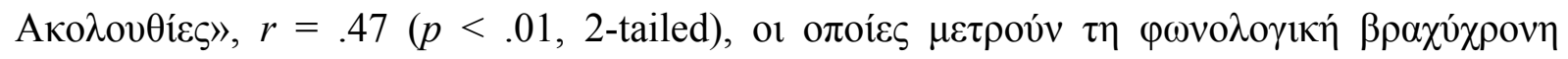
$\mu v \eta \dot{\mu \eta}, \kappa \alpha \imath$

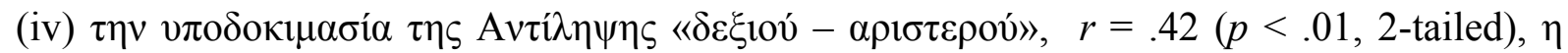

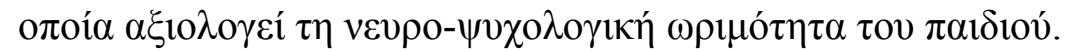

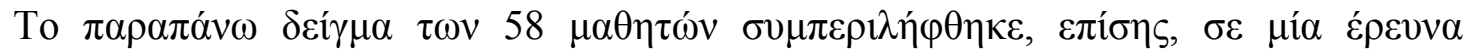

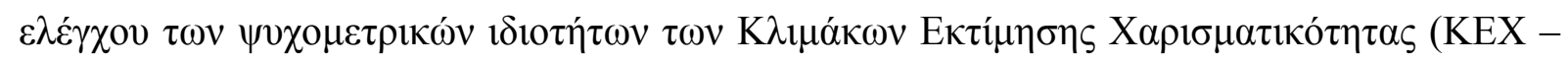

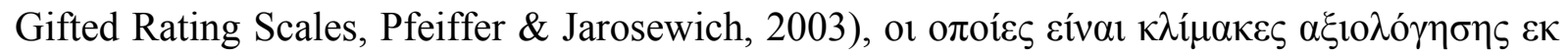

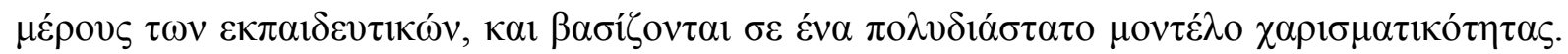

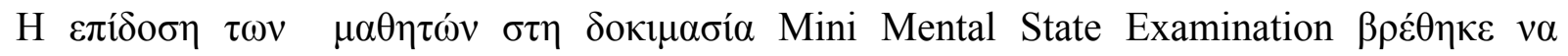

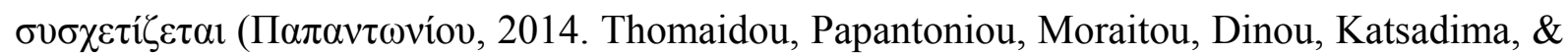

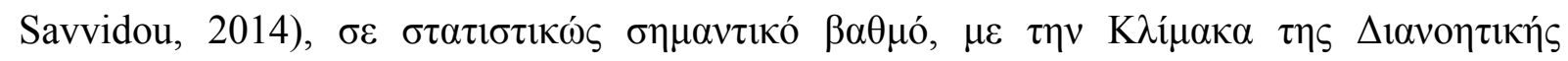

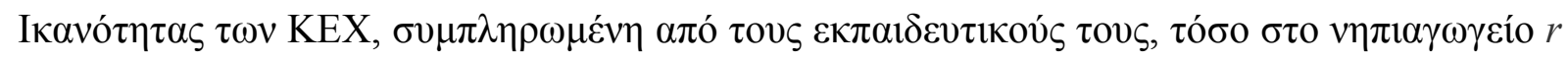

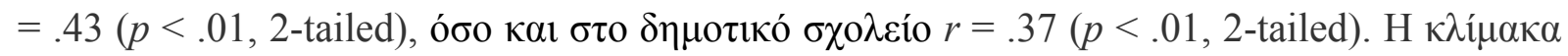

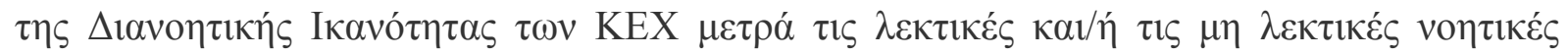

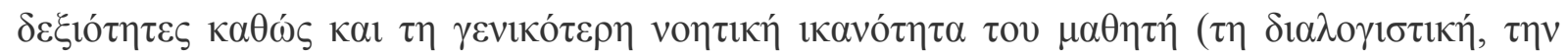

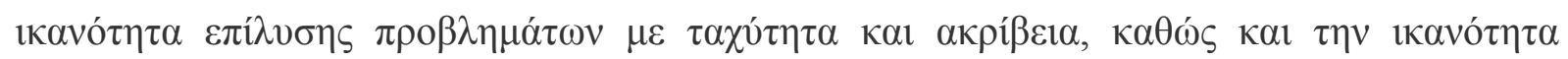

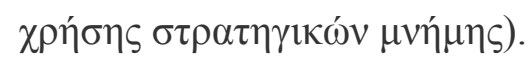




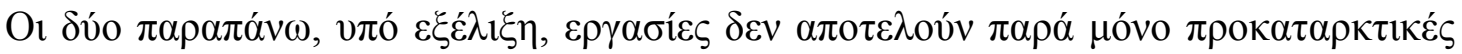

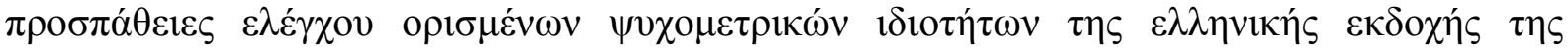

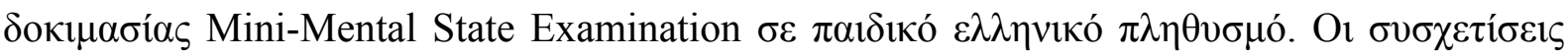

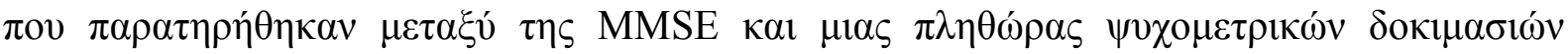

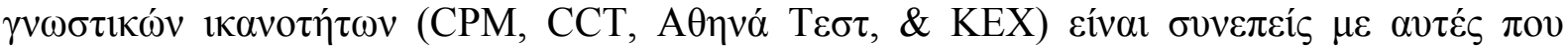

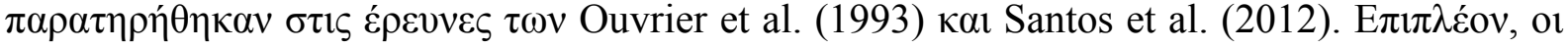

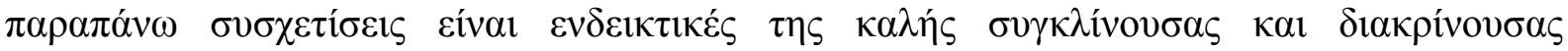

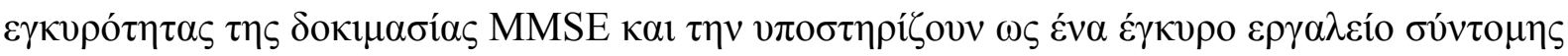

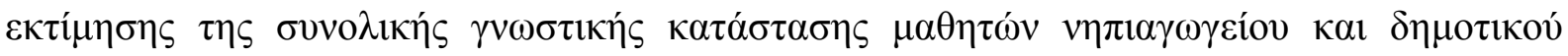

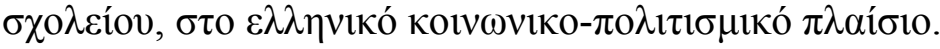




\section{ГYМПЕРАГМАТА}

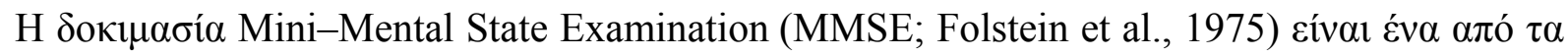

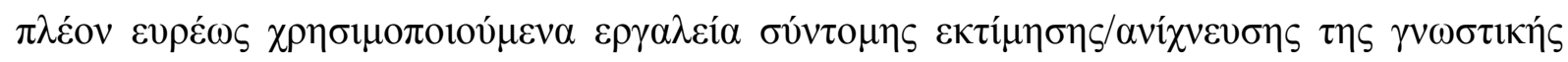

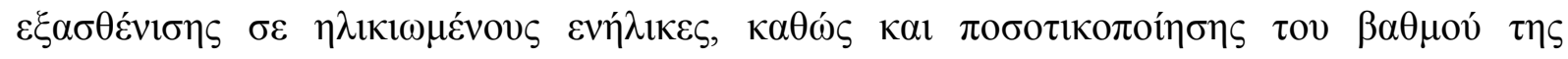

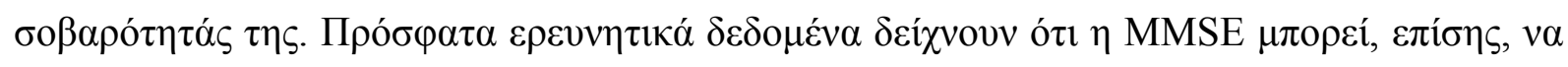

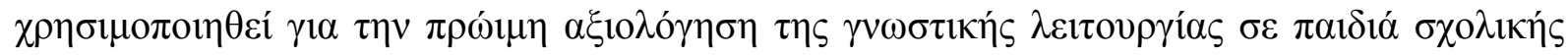

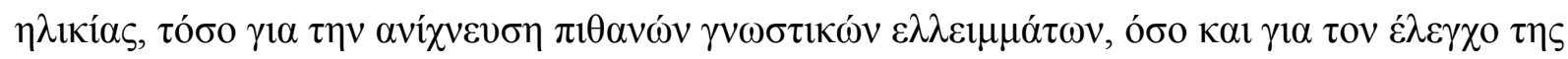

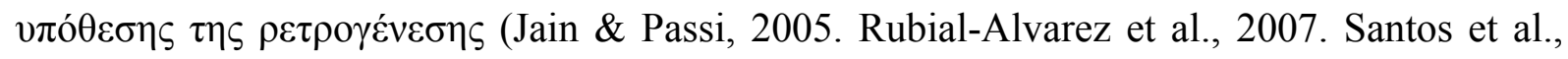

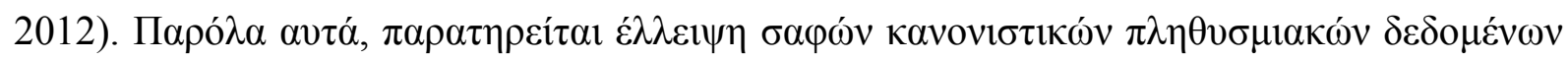

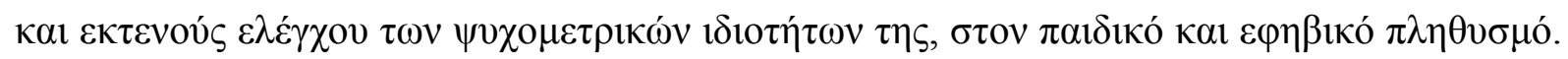

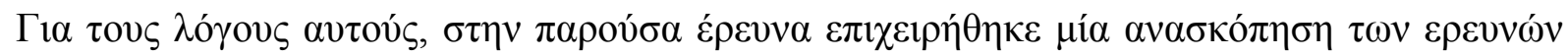

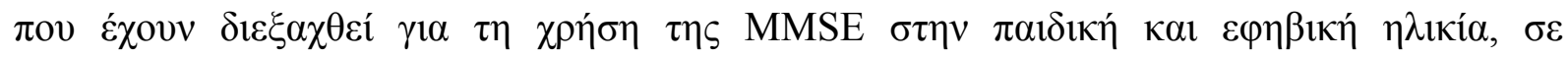

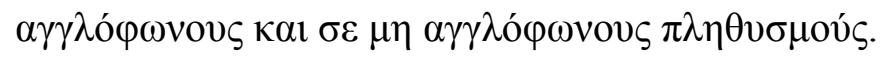

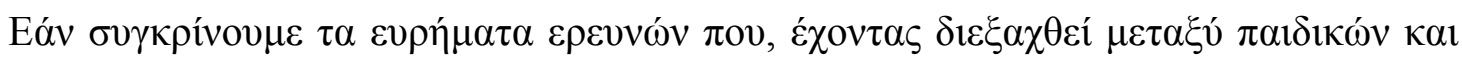

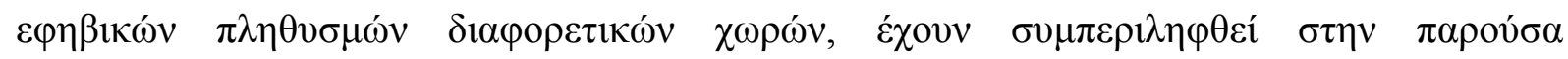

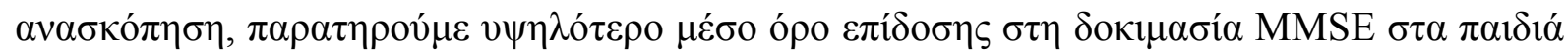

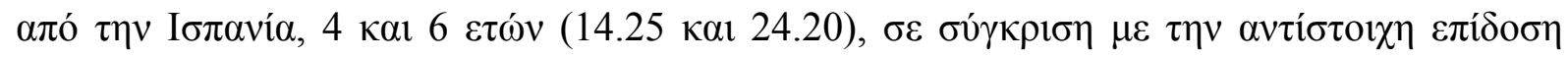

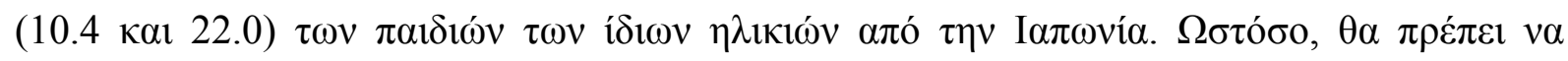

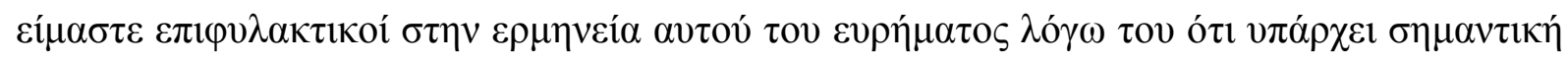

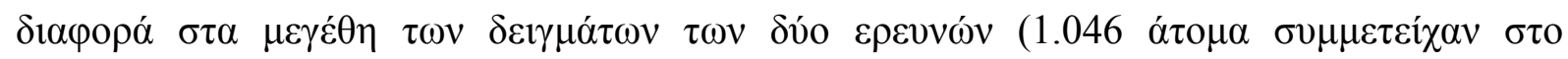

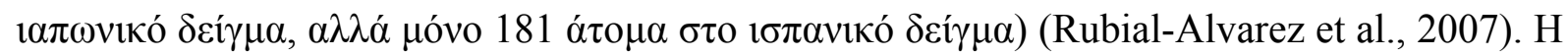

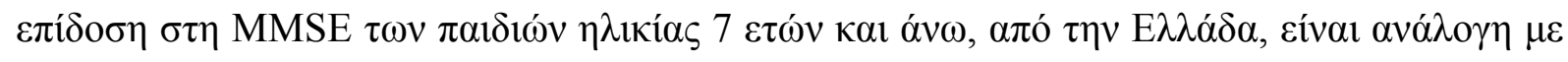

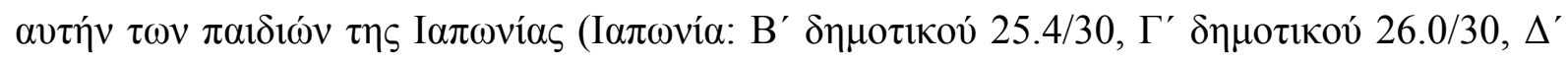




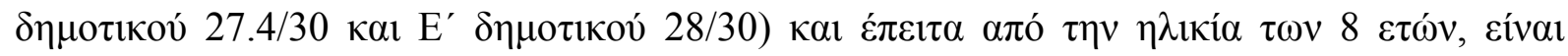

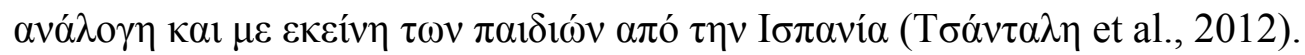

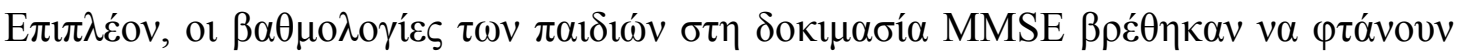

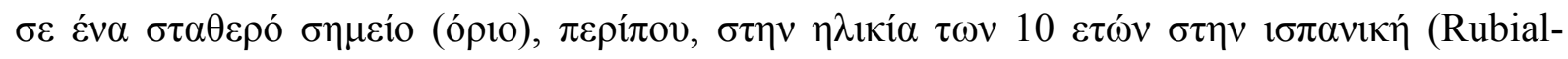

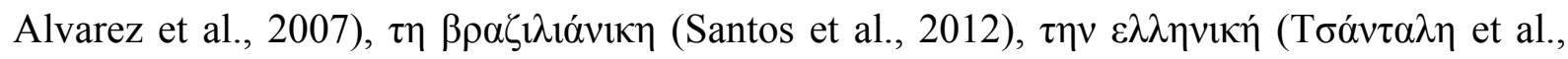

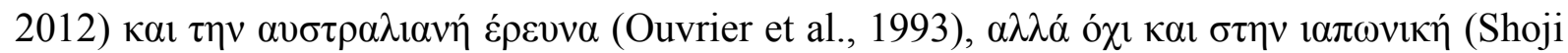

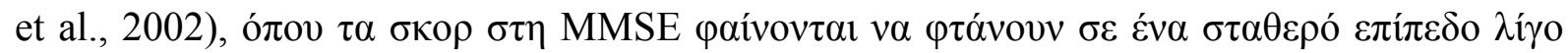

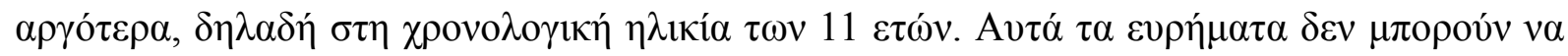

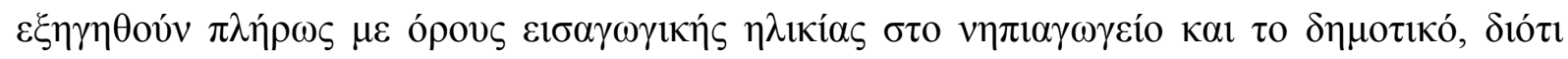

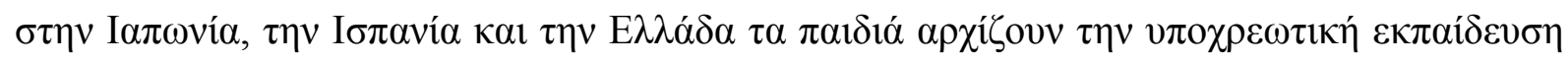

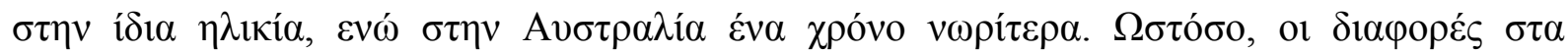

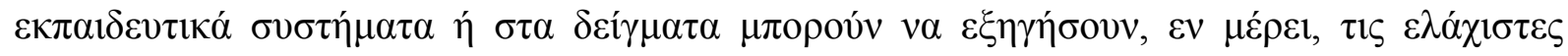

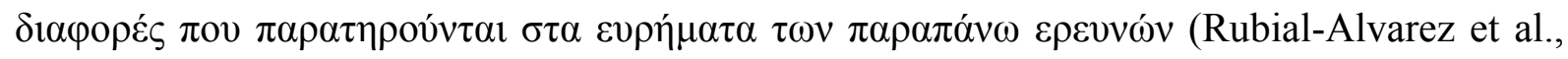
2007).

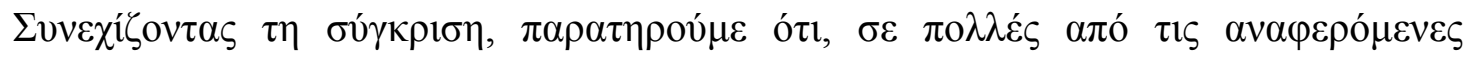

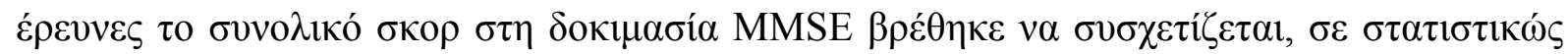

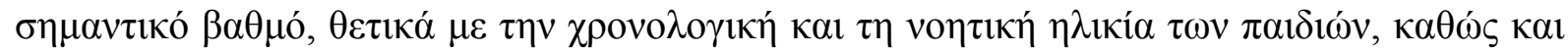

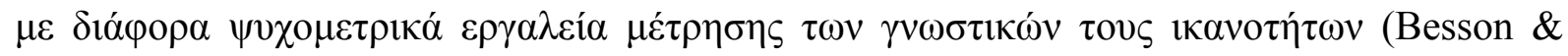
Labbe, 1997. Dinou et al., accepted. Ouvrier et al., 1993. Rubial-Alvarez et al., 2007. Santos

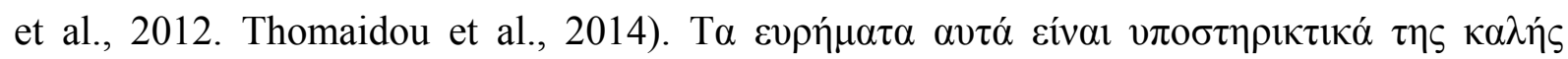

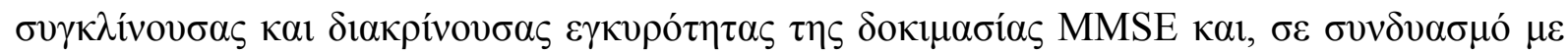

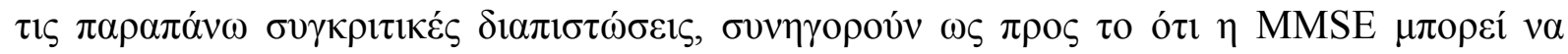

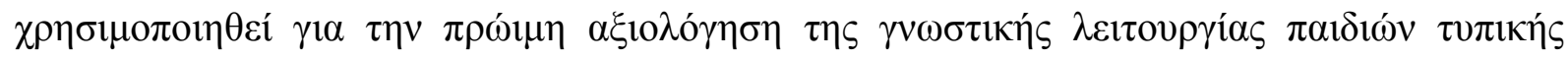

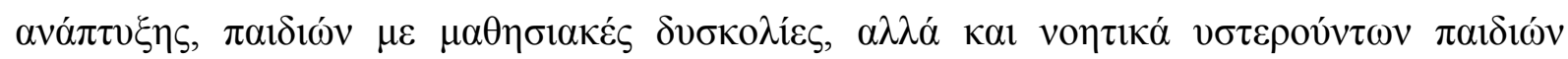


(Dinou et al., accepted. Jain \& Passi, 2005. Ouvrier et al., 1993. Rubial-Alvarez et al., 2007. Santos et al., 2012. Thomaidou et al., 2014).

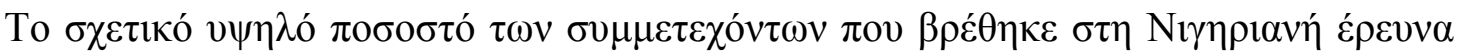

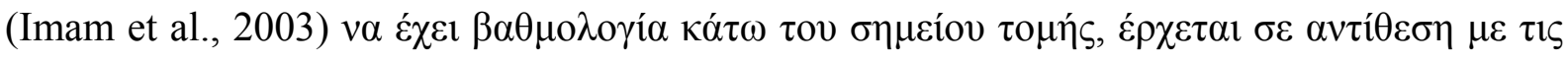

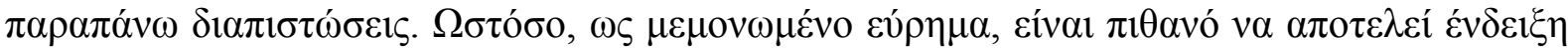

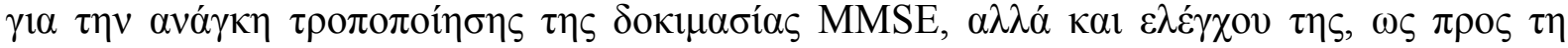

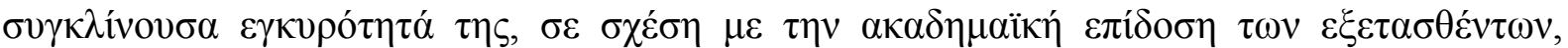

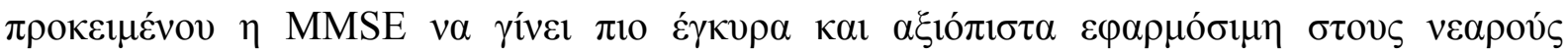

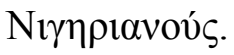

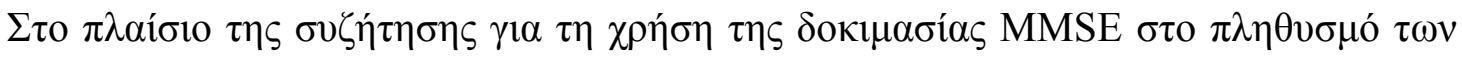

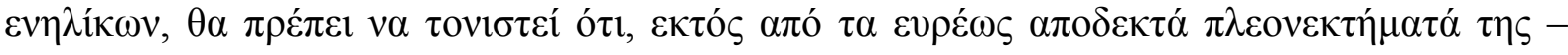

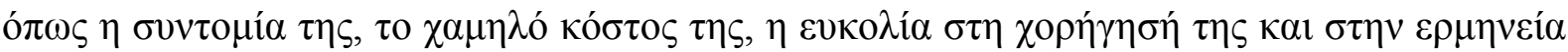

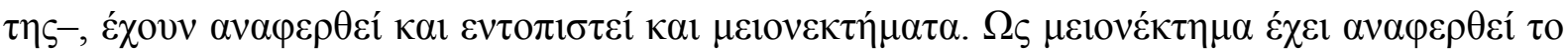

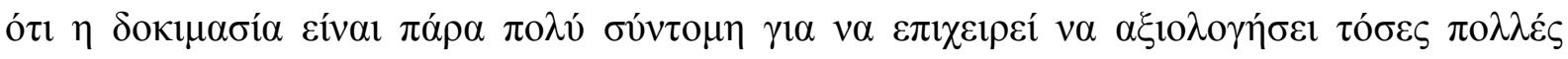

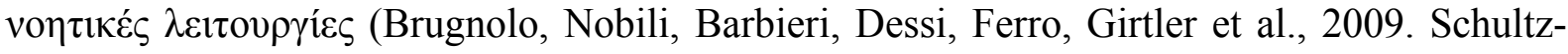
Larsen, Kreiner, \& Lomholt, 2007. Shigemori et al., 2010. Soubelet \& Salthouse, 2011)

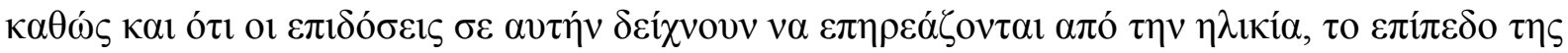

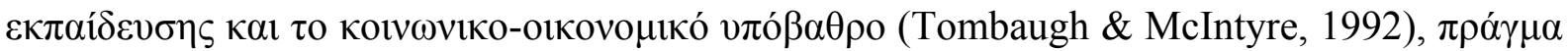

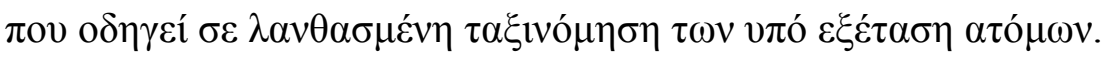

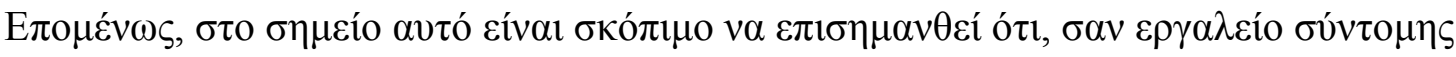

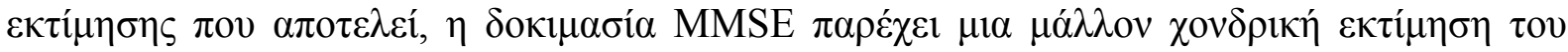

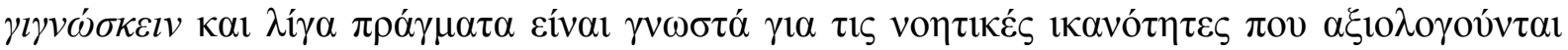

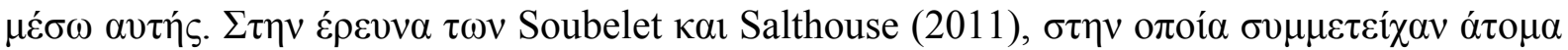

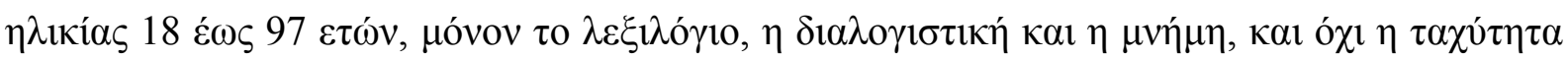

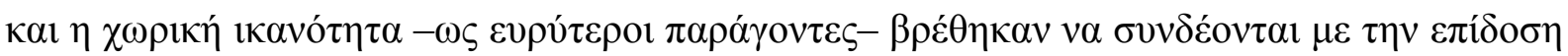




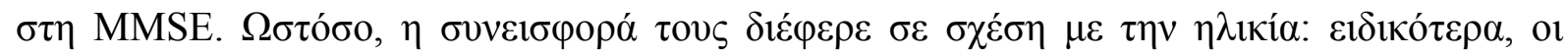

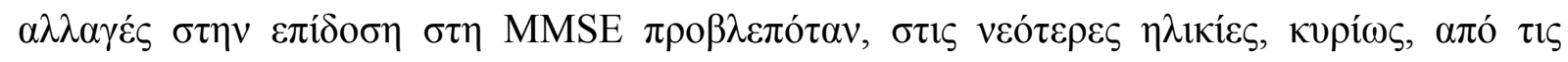

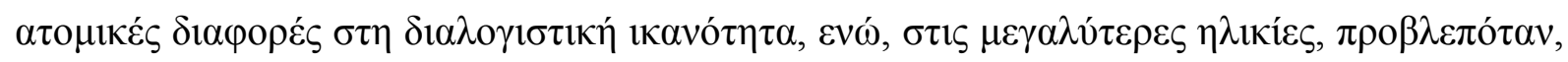

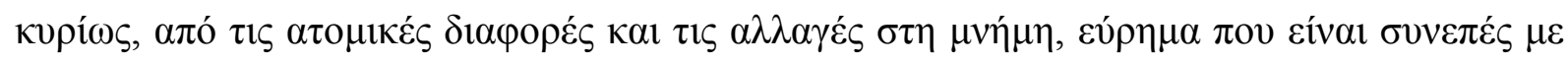

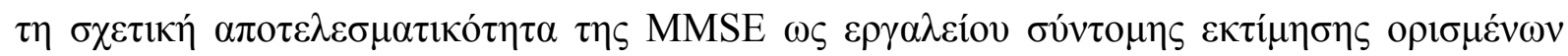

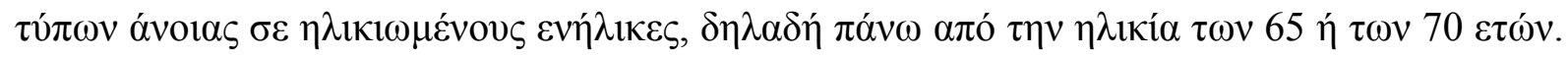

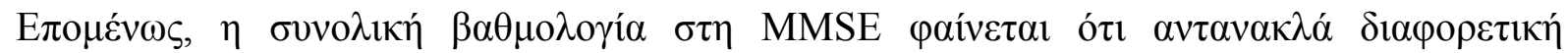

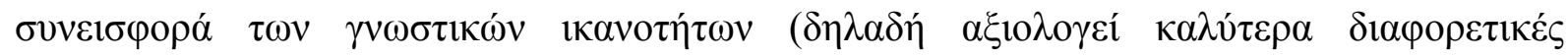

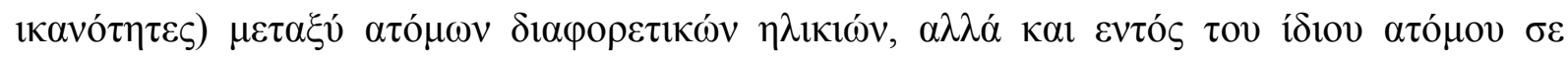

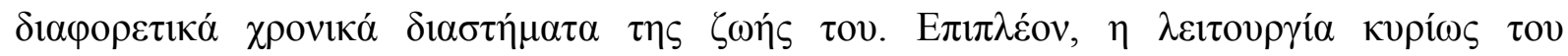

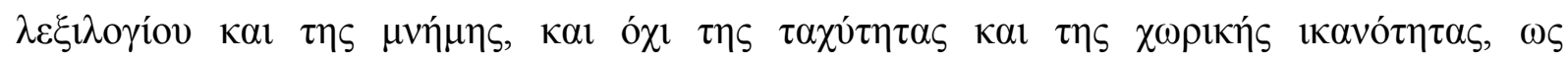

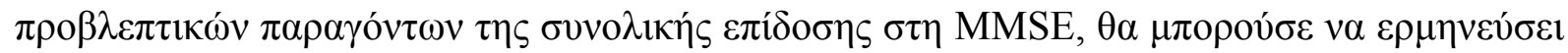

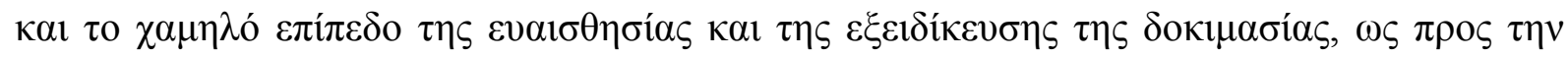

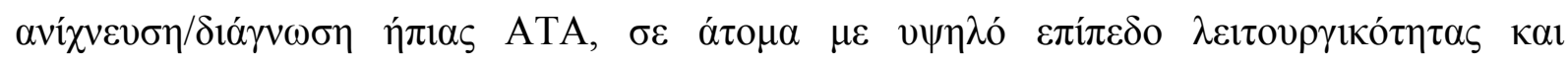
$\varepsilon \kappa \pi \alpha i ́ \delta \varepsilon v \sigma \eta \varsigma$.

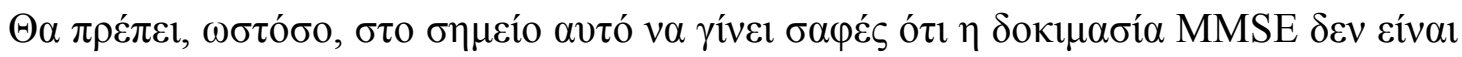

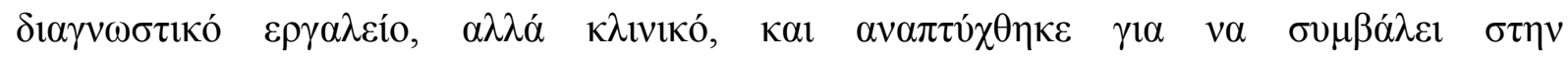

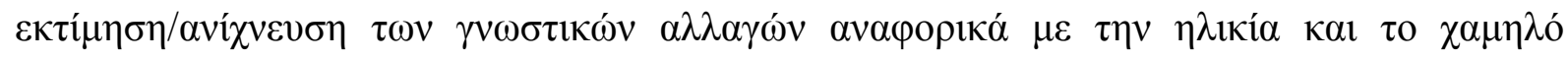

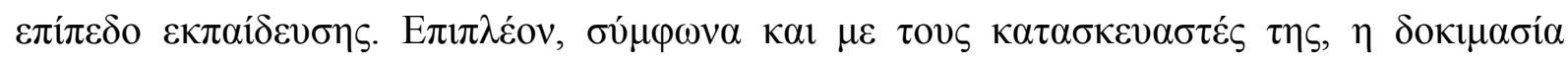

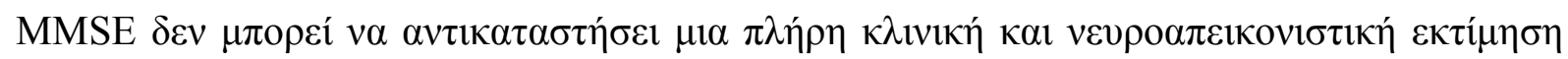

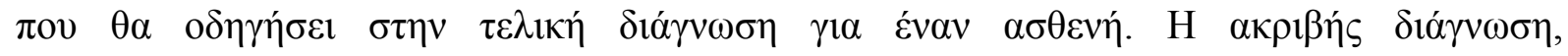

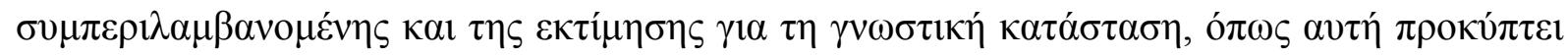

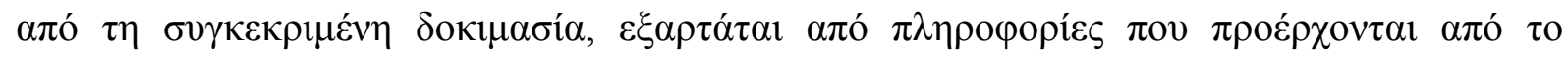

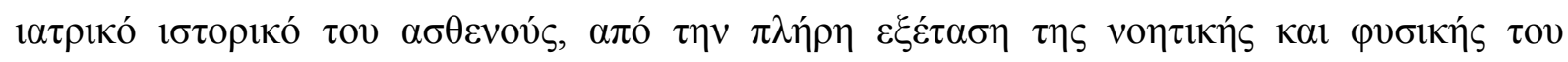

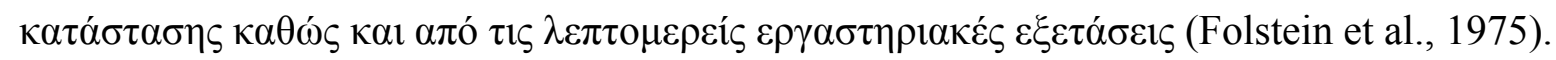




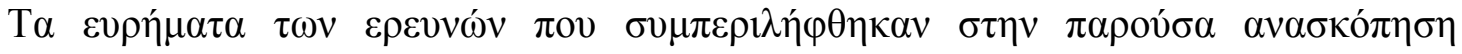

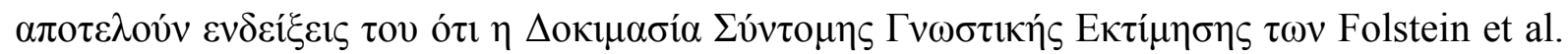

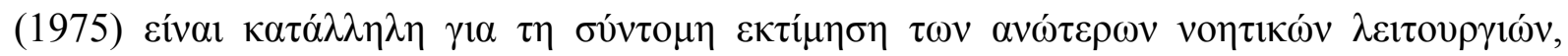

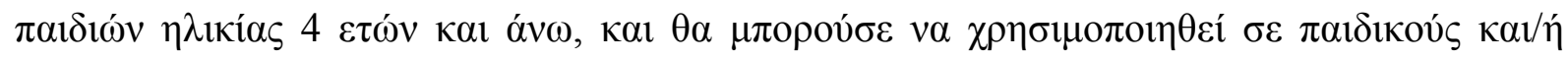

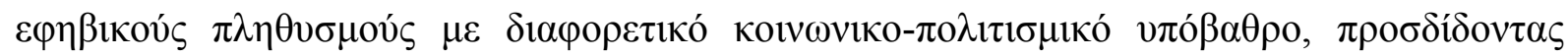
$\dot{\varepsilon} \gamma \kappa \cup \rho \alpha \alpha \pi$ $\alpha \varepsilon \lambda \varepsilon ́ \sigma \mu \alpha \tau \alpha$ (Dinou et al., accepted. Jain \& Passi, 2005. Ouvrier et al., 1993.

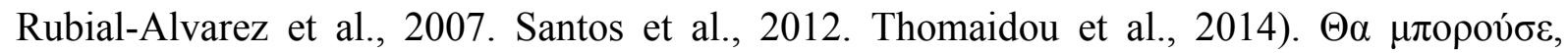

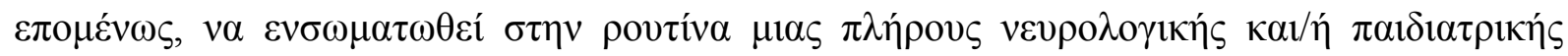

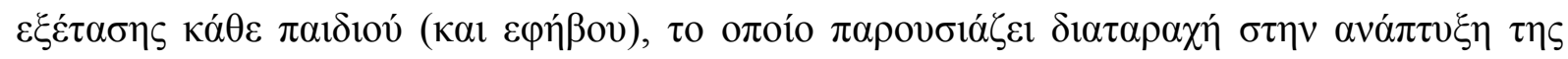

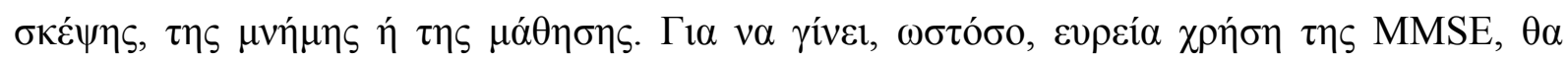

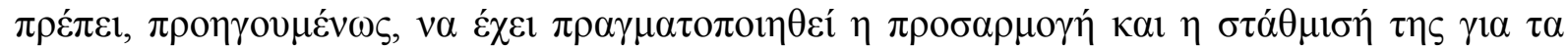

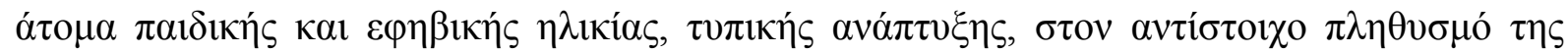

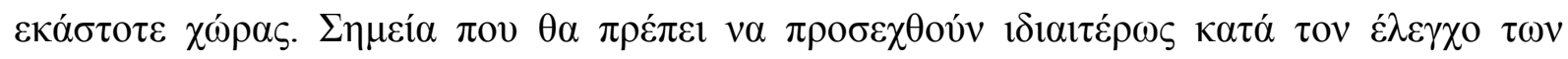

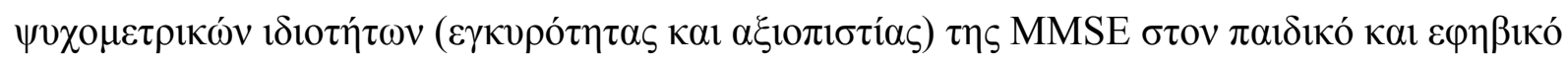

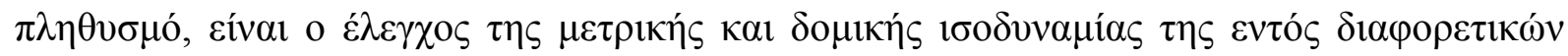

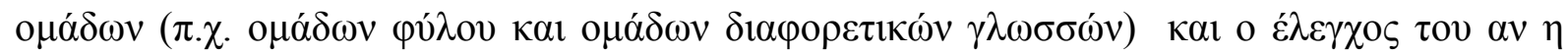

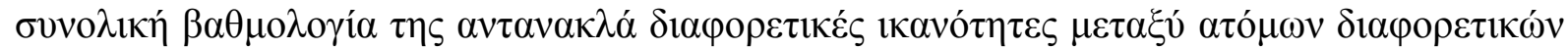

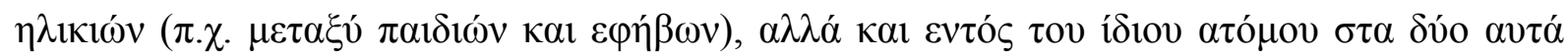

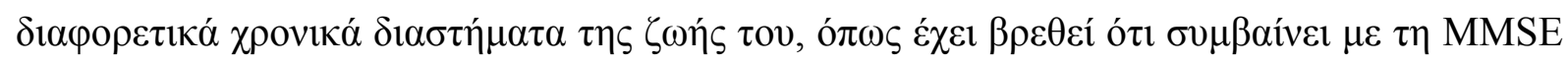

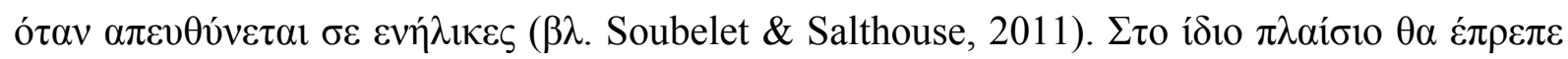

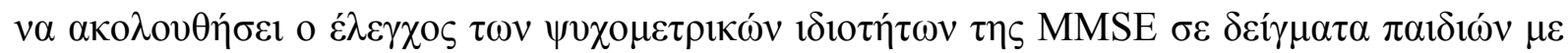

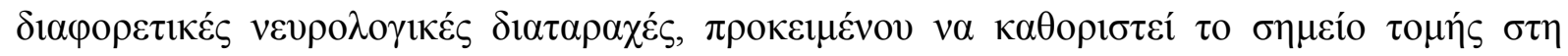

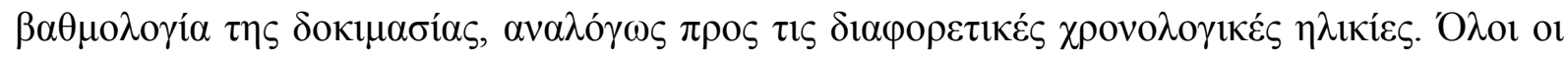

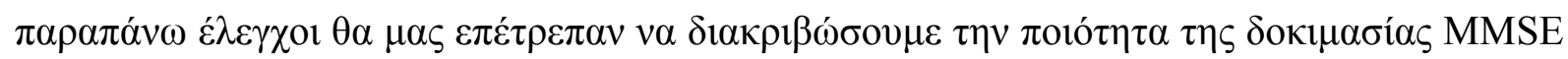




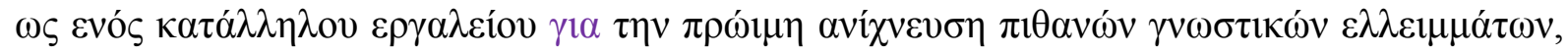

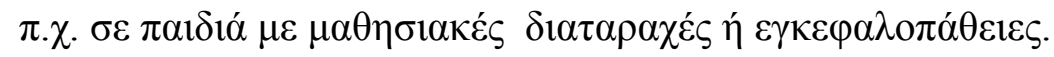

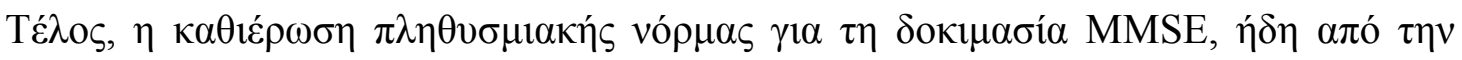

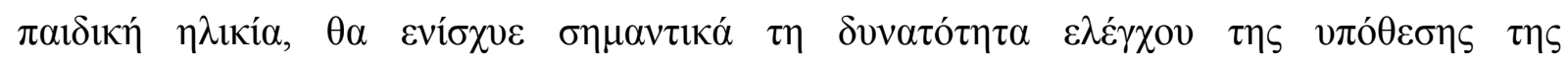

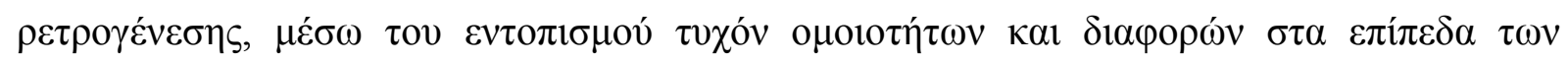

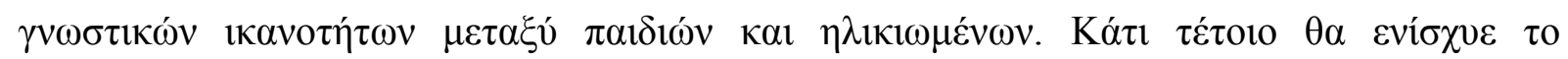

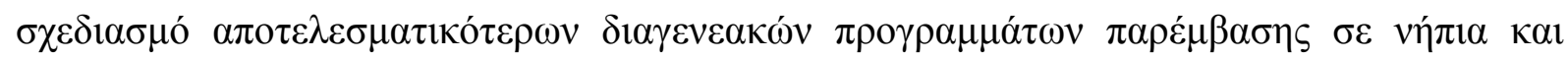

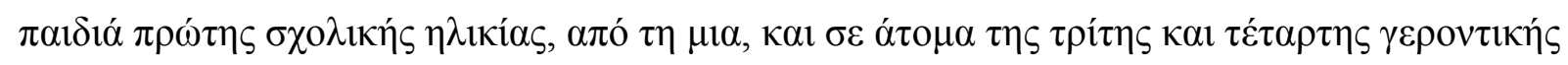

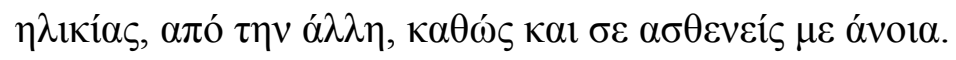




\section{ВIВАIОГРАФІА}

Achenbach, T. (1991). Manual for the Child Behavior Checklist/4 - 18 and 1991 Profile. Burlington, VT: University of Vermont Department of Psychiatry.

Albert, M., \& Cohen, C. (1992). The test for severe impairment: An instrument for the assessment of patients with severe cognitive dysfunction. Journal of American Geriatrics Society, 40(5), 449-453.

Beckman, K. M., \& Ames, B. N. (1998). The Free Radical Theory of Aging Matures. Physiological Reviews, 78(2), 547-81.

Besson, P. S., \& Labbe, E. E. (1997). Use of the modified Mini-Mental State Examination with children. Journal of Child Neurology, 12(7), 455-460.

Blesa, R., Pujol, M., Aguilar, M., Santacruz, P., Bertrán-Serra, I., Hernández, G., Sol, J. M., \& Peña-Casanova, J. (2001). Clinical validity of the "mini-mental state" for Spanish speaking communities, Neuropsychologia, 39, 1150-1157.

Boll, T. (1993). Manual for Children's Category Test. San Antonio, USA: Pearson.

Bornholt, L. J., Ajersch, S., Fisher, I. H., Markham, R. H., \& Ouvrier, R. A. (2010). Cognitive screening for children and adolescents: General limits or ceiling effects? Journal of Child Neurology, 25(5), 567-571.

Bornholt, L. J., Spencer, F. H., Fisher, I. H., \& Ouvrier, R. A. (2004). Cognitive screening for younger children: Development and diversity in learning contexts. Journal of Child Neurology, 19, 313-317.

Borza, L. R. (2012). The concept of retrogenesis: New ways of understanding Alzheimer's disease.Unpublished Dissertation, New York University, USA.

Brickman, A. M., Meier, I. B., Korgaonkar, M. S., Provenzano, F. A., Grieve, S. M., Siedlecki, K. L., Wasserman, B. T., Williams, L. M., \& Zimmerman, M. (2012). 
Testing the white matter retrogenesis hypothesis of cognitive aging. Neurobiology of Aging, 33(8), 1699-1715.

Brugnolo, A., Nobili, F., Barbieri, M. P., Dessi, B., Ferro, A., Girtler, N., Palummeri, E., Partinico, D., Raiteri, U., Regesta, G., Servetto, G., Tanganelli, P., Uva, V., Mazzei, D., Donadio, S., De Carli, F., Colazzo, G., Serrati, C., \& Rodriguez, G. (2009). The factorial structure of the mini mental state examination (MMSE) in Alzheimer's disease. Archives of Gerontology and Geriatrics, 49, 180-185.

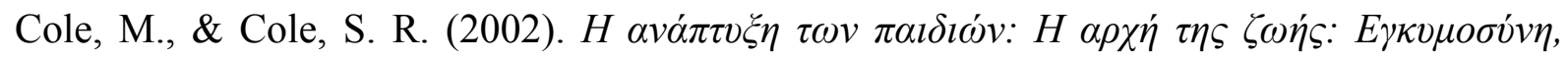

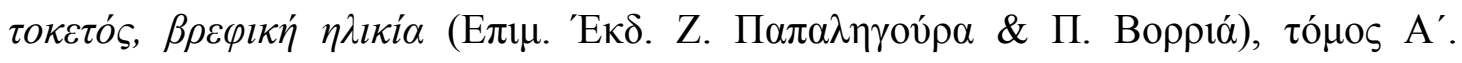

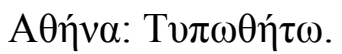

Da Silva, R., Bueno, O., \& Bertolucci, P. (2011). The retrogenesis theory to classify stages of Alzheimer's disease in a sample of the Brazilian population. Alzheimer's \& Dementia: The Journal of the Alzheimer's Association, 7(4), 241-242.

deAjuriaguerra, J., \&Tissot, R. (1968). Some aspects of psychoneurologic disintegration in senile dementia. In C. H. Mueller \& L. Ciompi (Eds.), Senile dementia (pp. 69-79). Switzerland: Huber.

De Mello, C. B., Abrisqueta-Gomez, J., Xavier, G. F., \& Bueno, O. F. A. (2008). Involution of catergorical thinking processes in Alzheimer's disease. Preliminary results. Dementia \& Neuropsychologia, 2(1), 57-62.

Dinou, M., Katsadima, E., Savvidou, E., Foutsitzi, E., Moraitou, D., \& Papantoniou, G. (accepted). Comparing the performance between Greek younger children and older adults on Mini-Mental State Examination: A preliminary study. In G. Papantoniou (Organizer \& Chair). Screening tests of general cognitive ability: Exploring their suitability for use with younger children and older adults. Round Table accepted at the $9^{\text {th }}$ Panhellenic Interdisciplinary Conference on Alzheimer's Disease and Related 
Disorders in combination with the $1^{\text {st }}$ Mediterranean Conference on Neurodegenerative Diseases, to be held on April 23-26, 2015, in Thessaloniki, Greece. Elliott, C. D. (1990). Differential Ability Scales: Administration and Scoring Manual. New York: The Psychological Corporation/Harcout Brace Jovanovich.

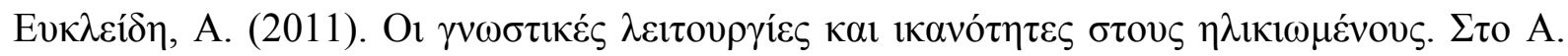

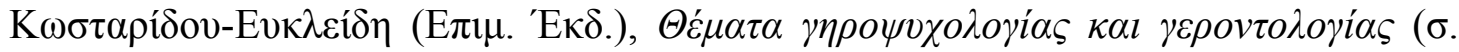

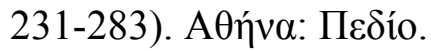

Ferrario, E., Seccia, L., Massaia, M., Fonte, G. F., \& Molaschi, M. (1998). Mini-Mental State Examination And Wechsler Memory Scale Subtest of Logical Memory: Correlation in an Over 70-Year-Old Population. Archives Gerontology Geriatric, 6, 175-180.

Folstein, M. F., Folstein, S. E., \& McHugh, P. R. (1975). Mini-mental state: A practical method for grading the cognitive state of patients for the clinicians. Journal of Psychiatric Research, 12, 189-198.

Imam, I., Onifade, A., Durodoye, M.O., Aje, A., Sogaolu, A., Kehinde, O., \& Ogunniyi, A. (2003). Performance of normal Nigerian students on the Mini-Mental State Examination. Nigerian Journal of Medicine, 12(3), 126-129.

Jain, M., \& Passi G. R. (2005). Assessment of a modified Mini-Mental Scale for cognitive functions in children. Indian Pediatrics, 42(9), 907-912.

Jones, R. N., \& Gallo, J. J. (2001). Education bias in the Mini-Mental State Examination. International Psychogeriatrics, 13(3), 299-310.

Kahle-Wrobleski, K., Corrada, M. M., Li, B., \& Kawas, C. H. (2007). Sensitivity and specificity of the Mini-Mental State Examination for identifying dementia in the oldest-old: The 90+ study. Journal of the American Geriatrics Society, 55(2), 284289. 
Lancu, I., \& Olmer, A. (2006). The Mini-Mental State Examination: An up-to-date review. Harefuah, the Journal of the Israeli Medical Association, 145(9), 687-690 [in Hebrew].

Matteson, M. A., Linton, A. D., \& Barnes, S. J. (1996). Cognitive developmental approach to dementia. Image: The Journal of Nursing Scholarship, 28(3), 233-240.

Moraitou, D., \& Efklides, A. (2012). The wise thinking and acting questionnaire: The cognitive facet of wisdom and its relation with memory, affect, and hope. Journal of Happiness Studies, 13(5), 849-873.

Morales, L., Flowers, C., Gutierrez, P., Kleinman, M., \& Tenesi, J. (2006). Item and scale differential functioning of the Mini-Mental State Exam assessed using the differential item and test functioning (DFIT) framework. Medical Care, 44, 143-151.

Ouvrier, R. A., Goldsmith, R. F, Ouvrier, S., \& Williams, I. C. (1993). The value of the MiniMental State Examination in childhood: A preliminary study. Journal of Child Neurology, 8(2), 145-148.

Ouvrier, R., Hendy, J., Bornholt, L., \& Black, F. (1999). The SYSTEMS: School-Years Screening Test for the evaluation of mental status. Journal of Child Neurology, 14, 772-780.

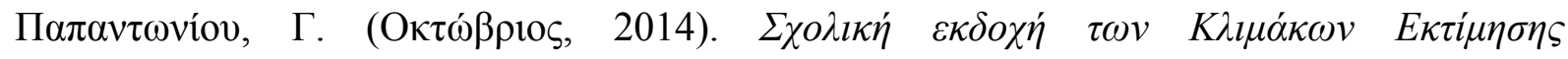

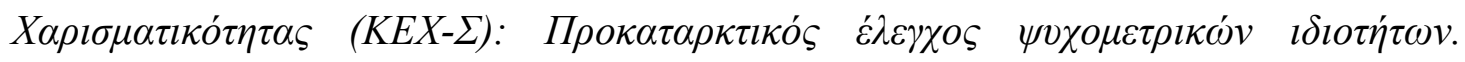

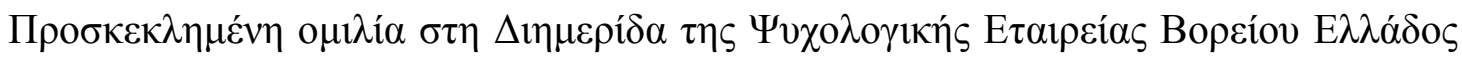

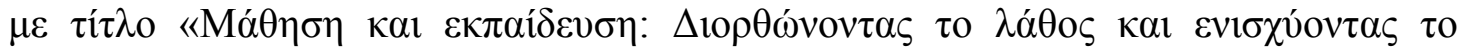
$\sigma \omega \sigma \tau o ́ », \Phi \lambda \omega ́ \rho ı v \alpha$.

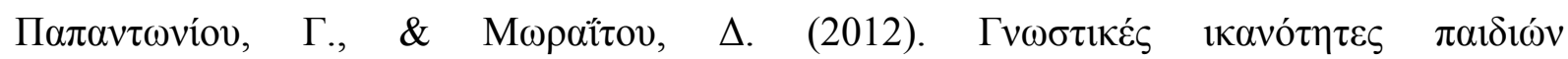

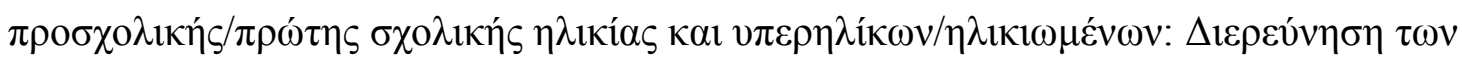




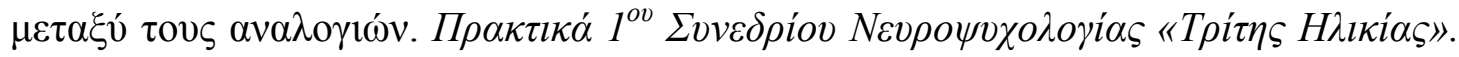

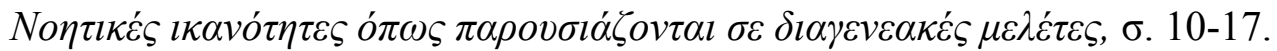

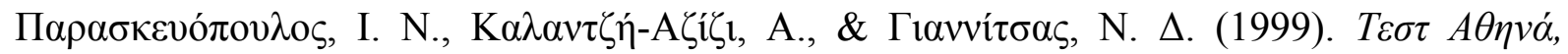

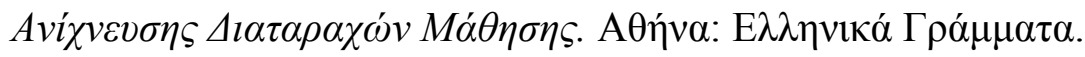

Peshawaria, R., \& Venkatesan, S. (1992). Behavioural Assessment Scales for Indian Children with Mental Retardation (BASIC-MR), NIMH. Secundrabad, 53-58.

Pfeiffer, S. I., \& Jarosewich, T. (2003). Gifted Rating Scales. San Antonio TX: The Psychological Corporation.

Piaget, J. (1952). The origins of intelligence in children. New York: International University Press.

Ramirez, M., Teresi, J. A., Holmes, D., Gurland, B., \& Lantigua, R. (2006). Differential Item Functioning (DIF) and the Mini-Mental State Examination (MMSE): Overview, sample and issues of translation. Medical Care, 44(11), 95-106.

Reinsberg, B., Franssen, E. H., Hasan, S. M., Monteiro, I., Boksay, I., Souren, L. E. M., Kenowsky, S., Auer, S. R., Elahi, S., \& Kluger, A. (1999a). Retrogenesis: clinical, physiologic, and pathologic mechanisms in brain aging, Alzheimer's and other dementing processes. European Archives of Psychiatry and Clinical Neuroscience, $249,28-36$.

Reinsberg, B., Franssen, E. H., Souren, L. E. M., Auer, S. R., Akram, I., \& Kenowsky, S. (2002). Evidence and mechanisms of retrogenesis in Alzheimer's and other dementias: Management and treatment import. American Journal of Alzheimer's Disease \& Other Dementias, 17, 202-212.

Reinsberg, B., Kenowsky, S., Franssen, E. H., Auer, S. R., \& Souren, L. E. M. (1999b). Towards a science of Alzheimer's disease management: A model based upon current knowledge of retrogenesis. International Psychogeriatrics, 11(1), 7-23. 
Rubial-Alvarez, S., Machado, M-C., Sintas, E., de Sola, S., Böhm, P., \& Peña-Casanova, J. (2007). A preliminary study of the Mini-Mental State Examination in a Spanish child population. Journal of Child Neurology, 22(11), 1269-73.

Rudial-Alvarez, S., Sola, S., Machado, M. C., Sintas, E., \& Bohm, P. (2013). The comparison of cognitive and functional performance in children and Alzheimer's disease supports the retrogenesis model. Journal of Alzheimer's Disease, 33(2), 191-203.

Rust, J. (2008). Manual for Raven's Coloured Progessive Matrices and Crichton Vocabulary Scale. San Antonio, USA: Pearson.

Salthouse, T. A., Pink, J. E., \& Tucher-Drob, E. M. (2008). Contextual analysis of fluid intelligence. Intelligence, 36, 464-486. doi: 10.1016/j.intell.2007.10.003

Santos, L. H. C., Pimentel, R. F., Rosa, L. G. D., Muzzolon, S. R. B., Antoniuk, S. A., \& Bruck, I. (2012). Cognitive and behavioral screening in children with learning disabilities: A preliminary study. Revista Paulista de Pediatra, 30(1), 93-99 [in English].

Scantlebury, N., Cunningham, T., Dockstader, C., Laughlin, S., Gaetz, W., Rockel, C., Dickson, J., \& Mabbot, D. (2014). Relation between white matter maturation and reaction time in childhood. Journal of the International Neuropsychological Society, 20, 99-112.

Scazufka, M., Almeida, O. P., Vallada, H. P., Tasse, W. A., \& Menezes, P. R. (2009). Limitations of the Mini-Mental State Examination for screening dementia in a community with low socioeconomic status: Results from the Sao Paulo Ageing and Health Study. European Archives of Psychiatry and Clinical Neuroscience, 259(1), 815. 
Schultz-Larsen, K., Kreiner, S., \& Lomholt, R. K. (2007). Mini-Mental Status Examination: Mixed Rasch model item analysis derived two different cognitive dimensions of the MMSE. Journal of Clinical Epidemiology, 60, 268-279.

Shigemori, K., Ohgi, S., Okuyama, E., Shimura, T., \& Schneider, E. (2010). The factorial structure of the mini mental state examination (MMSE) in Japanese dementia patients. BMC Geriatrics, 10, 36. doi: 10.1186/1471-2318-10-36

Shimada, M., Hayat, J., Meguro, K., Oo, T., Jafri, S., Yamadori, A., Franssen, E. H., \& Reisberg, B. (2003). Correlation between functional assessment staging and the 'Basic Age' by the Binet scale supports the retrogenesis model of Alzheimer's disease: a preliminary study. Psychogeriatrics, 3, 82-87.

Shoji, M., Fukushima, K., Wakayana, M., Shizuka-Ikeda, M., Ikeda , Y., Kawakami, A., Sakazume, Y., Ikeda, M., Harigaya, Y., Matsubara E., Kawarabayashi T., Murakami, T., Nagano, I., Manabe, Y., \& Abe, K. (2002). Intellectual faculties in patients with Alzheimer's disease regress to the level of a 4-5-year-old child. Geriatrics \& Gerontology International, 2(3), 143-147. 36.

Soubetet, A., \& Salthouse, T. A. (2011). Correlates of level and change in the Mini-Mental State Examination. Psychological Assessment, 23(4), 811-818.

Thomaidou, C., Papantoniou, G., Moraitou, D., Dinou, M., Katsadima, E., \& Savvidou, E. (2014, August 25-27). Reliability and concurrent validity of the Greek Version of the Preschool/Kindergarten Form of the Gifted Rating Scales (GRS-P). Poster presented at the $3^{\text {rd }}$ Biennial EARLI Conference of Special Interest Group 5 - Learning and Development in Early Childhood, Jyvaskyla, Finland.

Thorndike, R. L., Hagen, E. P., \& Sattler, J. M., (1986). Stanford-Binet Intelligence Scale 4th Edition. Chicago: DLM Teaching Resources-Riverside. 
Tombaugh, T., \& McIntyre, N. (1992). The mini-mental state examination: A comprehensive review. Journal of the American Geriatrics Society, 40(9), 922-935.

Tsantali, E., Economides, D., \& Rigopoulou, S. (May, 2009). Comparison of cognitive profile of normal children, middle aged and elderly people with and without cognitive impairements. Paper presented at the $14^{\text {th }}$ International Conference of the Association of Psychology and Psychiatry for Adults and Children, Athens, Greece.

Tsantali, E., Economidis, D., Rigopoulou, S., \& Porpodas, C. (2012). Comparison of cognitive performance in mild cognitive impairment and dementia patients with that in normal children and adults. Geriatrics and Gerontology International, 12, 336-344.

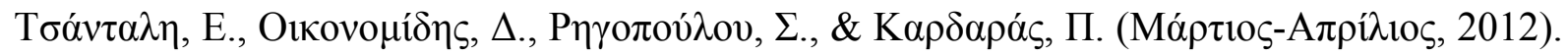

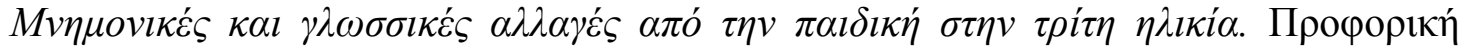

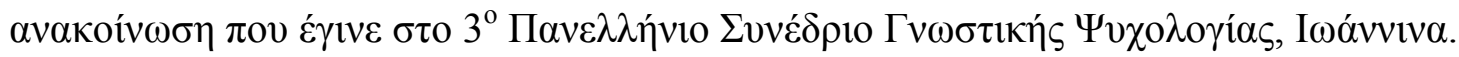

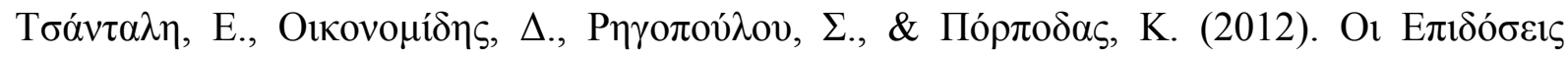

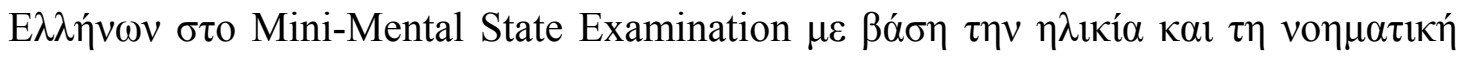

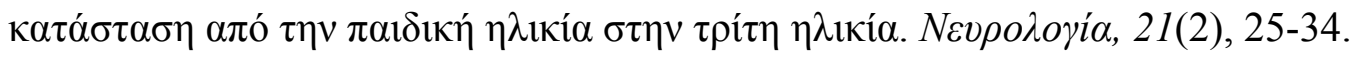

Tsolaki, M., Fountoulakis, K., Nakopoulou, E., Kazis, A., \& Mohs, R. C. (1997). Alzheimer's Disease Assessment Scale: The validation of the scale in Greece in elderly demented patients and normal subjects. Dementia and Geriatric Cognitive Disorders, 8(5), 27380.

Ventirelli, M., Magalioni, A., Scarcini, R., \& Schena, F. (2012). From Alzheimer's Disease Retrogenesis A New Strategy for Patients With Advanced Dementias. American Journal of Alzheimer's Disease and Other Dementias, 27(7), 483-489.

Wechsler, D. (1989). Wechsler Preschool and Primary Scale of Intelligence - Revised. New York: The Psychological Corporation/Harcout Brace Jovanovich. 
Wechsler, D. (1991). Wechsler Intelligence Scale for Children - Third Edition. New York: The Psychological Corporation/Harcout Brace Jovanovich. 